Supporting Information for

\title{
Catalytic Electrophilic Thiocarbocyclization of Allenes
}

\author{
Quanbin Jiang, ${ }^{\dagger}$ Huimin $\mathrm{Li}^{\dagger}{ }^{\dagger}$ and Xiaodan Zhao* \\ Institute of Organic Chemistry \& MOE Key Laboratory of Bioinorganic and Synthetic Chemistry \\ School of Chemistry, Sun Yat-Sen University, Guangzhou 510275, China \\ E-mail: zhaoxd3@mail.sysu.edu.cn
}

\section{Table of Contents}

1. General considerations

2. Procedures for the preparation of racemic allene substrates and sulfur S3 reagents

2.1 Synthesis of racemic allene substrates

2.2 Synthesis of sulfur reagents

3. General procedure for the catalytic reactions

4. Characterization data

5. References 


\section{General considerations}

Unless otherwise stated, commercial reagents were purchased from Alfa, Macklin, Energy or Adamas, and used without further purification. Reaction solvents THF and $\mathrm{CH}_{2} \mathrm{Cl}_{2}$ were purified by PureSolv MD-5 (Innovative Technology). Super dry DMF were purchased from $J \& K$. Catalytic reactions were carried out using pre-dried glassware without special care. Analytical thin layer chromatography was performed on $0.20 \mathrm{~mm}$ silica gel HSGF-254 plates (Huanghai, China), and visualized under 254 nm UV light or by staining with potassium permanganate. Preparative thin layer chromato graphy was performed on 0.4-0.5 mm silica gel HSGF-254 plates (Huanghai, China). Column chromatography was performed on 200-300 mesh silica gel (Huanghai, China). Catalysts $\mathbf{C 1},{ }^{1} \mathbf{C 2},{ }^{1} \mathbf{C} 3,{ }^{1}$ and $\mathbf{C} 4{ }^{2}$ were synthesized according to the literature methods. Catalysts $\mathrm{PhSPh}$ and $\mathrm{Ph}_{3} \mathrm{P}=\mathrm{S}$ are commercially available, and $\mathrm{Ph}_{3} \mathrm{P}=\mathrm{Se}$ was synthesized by using $\mathrm{Se}$ and triphenyl phosphine.

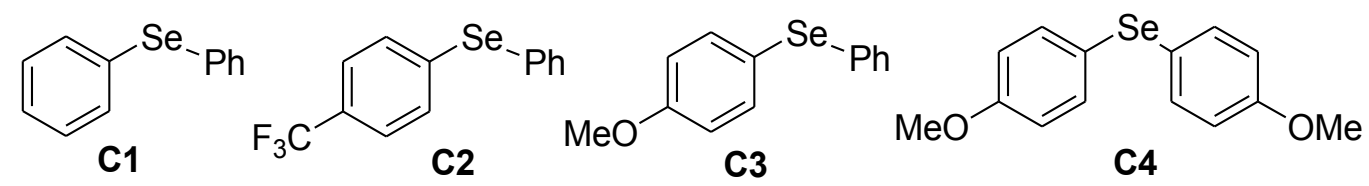

${ }^{1} \mathrm{H},{ }^{13} \mathrm{C}$ and ${ }^{19} \mathrm{~F}$ NMR spectra were recorded on Brucker ARX $400 \mathrm{MHz}$ spectrometer at ambient temperature. All NMR spectra are referenced to the residual solvent signal. Data for ${ }^{1} \mathrm{H}$ NMR are reported as follows: chemical shift ( $\left.\delta \mathrm{ppm}\right)$, multiplicity $(\mathrm{s}=$ singlet, $\mathrm{d}=$ doublet, $\mathrm{t}=$ triplet, $\mathrm{q}=$ quartet, $\mathrm{m}=$ multiplet $)$, coupling constant $(\mathrm{Hz})$, integration. Data for ${ }^{13} \mathrm{C}$ NMR are reported as follows: chemical shift $(\delta$ ppm), multiplicity $(\mathrm{d}=$ doublet, $\mathrm{t}=$ triplet, $\mathrm{q}=$ quartet $)$, coupling constant $(\mathrm{Hz})$. High resolution mass spectra of new compounds were recorded on LTQ Orbitrap Elite LC/MS (ESI or APCI) at analytical center of Sun Yat-Sen University, and Guangzhou Micromon Technology Services CO. LTD. 


\section{Procedures for the preparation of racemic allene substrates and}

\section{sulfur reagents}

\subsection{Synthesis of racemic allene substrates}<smiles>C/C(=C\c1ccccc1)c1ccccc1</smiles><smiles>CC(=Cc1ccc(Cl)cc1)c1ccccc1</smiles>

$1 d$<smiles>COc1ccccc1C=C(C)c1ccccc1</smiles><smiles>CC(=Cc1ccccc1)c1ccc(Cl)cc1</smiles>

$1 \mathrm{j}$<smiles>C(=C/c1ccccc1)\c1ccccc1</smiles><smiles>CC/C(=C\c1ccccc1)c1ccccc1</smiles><smiles>C/C(=C\c1ccc(C)cc1)c1ccccc1</smiles><smiles>C/C(=C\c1ccc(F)cc1)c1ccccc1</smiles><smiles>CC(=C=Cc1ccccc1)c1ccc(C)cc1</smiles><smiles>CC(=Cc1ccccc1)c1ccc(F)cc1</smiles>

$1 \mathrm{k}$<smiles>CCCC=C=C(c1ccccc1)c1ccccc1</smiles>

1n<smiles>COc1cccc(C=C(C)c2ccccc2)c1</smiles><smiles>C/C(=C\c1ccc(Br)cc1)c1ccccc1</smiles><smiles>COc1ccc(C=C=C(C)c2ccccc2)cc1</smiles><smiles>CC(=Cc1ccccc1)c1ccc(C#N)cc1</smiles><smiles>CC(=Cc1ccccc1)c1cccc(F)c1</smiles>

11<smiles>CCCCCC=C=C(CC)c1ccccc1</smiles><smiles>CCCCC=C=C(C)c1ccccc1</smiles>

Method A:

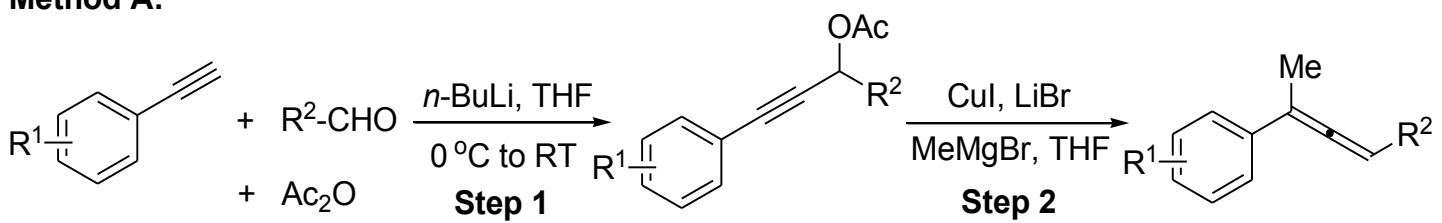

Method B:<smiles>[R]C=C(CC)C(CC)c1cc[R1]([H])cc1</smiles>

Method C:

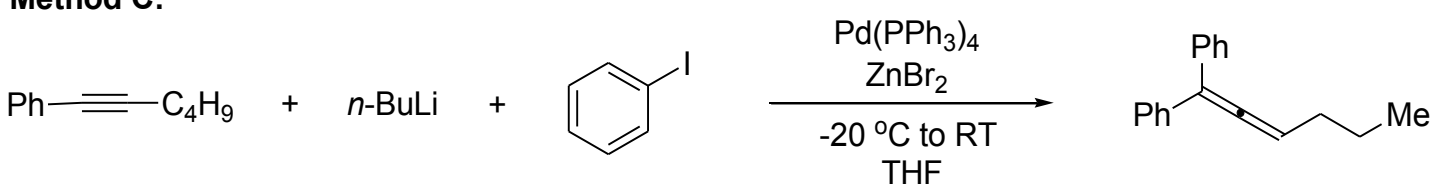




\section{Method A:}

General procedure for the synthesis of allenes was based on the known procedure. ${ }^{3}$ Step 1: To a THF $(1 \mathrm{M})$ solution of aryl acetylene (1.0 equiv) was added $n$-BuLi (1.0 equiv) at $0{ }^{\circ} \mathrm{C}$ under $\mathrm{N}_{2}$ atmosphere, and the mixture was stirred at $0{ }^{\circ} \mathrm{C}$ for $30 \mathrm{~min}$ before it was treated with aldehyde (1.0 equiv). The mixture was stirred for $30 \mathrm{~min}$ at room temperature and then acetic anhydride (1.6 equiv) was added to the flask at $0{ }^{\circ} \mathrm{C}$, and the mixture was stirred for another $2 \mathrm{~h}$ at room temperature before adding water. The aqueous solution was extracted with EtO Ac $(15 \mathrm{~mL}$ x 3). The combined organic layers were dried over anhydrous $\mathrm{Na}_{2} \mathrm{SO}_{4}$, filtered, and concentrated in vacuo. The residue was purified by silica gel column chromatography to afford the propargyl acetate for the next step.

Step 2: To a $50 \mathrm{~mL}$ oven-dried Schlenk flask were added $\mathrm{CuI}$ (2.0 equiv), $\mathrm{LiBr}$ (2.0 equiv), THF (0.25 M), and then $\operatorname{MeMgBr}\left(3 \mathrm{M}\right.$ in $\mathrm{Et}_{2} \mathrm{O}, 2.0$ equiv) under $\mathrm{N}_{2}$ atmosphere at $0{ }^{\circ} \mathrm{C}$. The mixture was stirred for $30 \mathrm{~min}$ at room temperature and then propargyl acetate was added at $0{ }^{\circ} \mathrm{C}$. The resulting mixture was stirred overnight at room temperature, and then poured into a beaker with a mixture of saturated aqueous $\mathrm{Na}_{2} \mathrm{CO}_{3}$ solution and $n$-hexane $(1: 1, \mathrm{v} / \mathrm{v})$. The mixture was filtered to remove the solid. The filtrate was extracted with EtOAc $(15 \mathrm{~mL}$ x 3$)$. The combined organic layers were dried over anhydrous $\mathrm{Na}_{2} \mathrm{SO}_{4}$, filtered, and concentrated in vacuo. The residue was purified by silica gel column chromatography to afford the final product.

\section{Method B:}

Step 1: The first step was the same to the step 1 of method A.

Step 2: This step was performed on the basis of the known procedure. ${ }^{4}$

To a $50 \mathrm{~mL}$ oven-dried Schlenk flask were added $\mathrm{Pd}(\mathrm{OAc})_{2}$ (0.05 equiv), SPhos (0.075 equiv), and anhydrous DMF (0.5 M) sequentially under $\mathrm{N}_{2}$ atmosphere. The resulting mixture was stirred at room temperature for $30 \mathrm{~min}$. Then, propargyl acetate (0.25 $\mathrm{M}$ in DMF, 1.0 equiv) and $\mathrm{Et}_{2} \mathrm{Zn}(1.0 \mathrm{M}$ in toluene, 2.0 equiv) were sequentially added to the flask at room temperature. After being stirred at room temperature for $4 \mathrm{~h}$, the resulting mixture was quenched with an aqueous solution of $3 \mathrm{M} \mathrm{HCl}$, and 
extracted with ethyl ether $(15 \mathrm{~mL} \times 3)$. The combined organic layers were washed with brine, dried over anhydrous $\mathrm{Na}_{2} \mathrm{SO}_{4}$, filtrated, and concentrated in vacuo. The residue was purified by column chromatography on silica gel (eluent: PE) to afford the allene.

\section{Method C:}

The procedure was based on the known procedure. ${ }^{5}$

$n$ - $\mathrm{BuLi}$ (1.2 equiv) was added to a solution of 1 -phenyl-1-hexyne (1.0 equiv) in THF $(0.2 \mathrm{M})$ in a dry Schlenk flask at $-20{ }^{\circ} \mathrm{C}$ under $\mathrm{N}_{2}$ atmosphere. The reaction mixture was stirred at $-20{ }^{\circ} \mathrm{C}$ for $1 \mathrm{~h}$. Then, a solution of dry $\mathrm{ZnBr}_{2}$ (2.0 equiv) in THF (0.15 M) was added. After a further $10 \mathrm{~min}$ at this temperature, the mixture was warmed up to room temperature and stayed for $20 \mathrm{~min}$. $\mathrm{Pd}\left(\mathrm{PPh}_{3}\right)_{4}(5 \mathrm{~mol} \%)$ and iodobenzene $(1.2$ equiv) were subsequently added, and the resulting mixture was stirred at room temperature. The reaction was monitored by TLC. When the reaction was complete, it was quenched with saturated $\mathrm{NH}_{4} \mathrm{Cl}$. The mixture was extracted with ether $(15 \mathrm{~mL} \mathrm{x}$ 3), and dried over anhydrous $\mathrm{Na}_{2} \mathrm{SO}_{4}$. The solvents were removed by rotary evaporation. The residue was purified by flash chromatography on silica gel (eluent: PE) to afford the product.

Compounds 1a-1 m and 1q-1r were synthesized by method A, $1 \mathbf{n}$ was synthesized by method C, and 1o-1p were synthesized by method B. Compounds $1 \mathbf{a},{ }^{3} 1 \mathbf{b},{ }^{6} 1 \mathbf{c},{ }^{7} 1 \mathrm{~d},{ }^{7}$ $\mathbf{1 f},{ }^{8} \mathbf{1 g},{ }^{7} \mathbf{1} \mathbf{h},{ }^{9} \mathbf{1} \mathbf{j},{ }^{9} \mathbf{1} \mathbf{m},{ }^{9} \mathbf{1 n},{ }^{10} \mathbf{1 p},{ }^{4} \mathbf{1 q},{ }^{9} \mathbf{1} \mathbf{r}^{3}$ are known and their spectral data are in accordance with the reported in the literature. Compounds $\mathbf{1 e}, \mathbf{1 i}, \mathbf{1 k}, \mathbf{1 l}$ and $\mathbf{1 0}$ are new.

\section{1-Fluoro-4-(3-phenylbuta-1,2-dien-1-yl)benzene (1e)}<smiles>CC(=Cc1ccc(F)cc1)c1ccccc1</smiles>

Prepared by the Method A: Phenylacetylene (1100 $\mu \mathrm{L}, 10 \mathrm{mmol}, 1.0$ equiv), $n$-BuLi 
(4.2 mL, 2.4 $\mathrm{M}$ in hexane, 1.0 equiv), 4-fluorobenzaldehyde (1075 $\mu \mathrm{L}, 1.0$ equiv), $\mathrm{Ac}_{2} \mathrm{O}(1515 \mu \mathrm{L}, 1.6$ equiv) and THF $(10 \mathrm{~mL})$.

Step 1: The residue was purified by column chromatography on silica gel (eluent: $\mathrm{PE} / \mathrm{EtOAc}=30 / 1, \mathrm{v} / \mathrm{v}) .1389 .5 \mathrm{mg}, 52 \%$ yield, a light yellow oil.

Step 2: The residue was purified by column chromatography on silica gel (eluent: PE). $810.3 \mathrm{mg}, 70 \%$ yield, a colorless oil.

${ }^{1} \mathrm{H}$ NMR (400 MHz, $\left.\mathrm{CDCl}_{3}\right) \delta 7.58$ - $7.55(\mathrm{~m}, 2 \mathrm{H}), 7.46-7.42(\mathrm{~m}, 2 \mathrm{H}), 7.40-7.32$ (m, 3H), $7.12-7.07(\mathrm{~m}, 2 \mathrm{H}), 6.55(\mathrm{q}, J=2.9 \mathrm{~Hz}, 1 \mathrm{H}), 2.34(\mathrm{~d}, J=3.0 \mathrm{~Hz}, 3 \mathrm{H})$.

${ }^{13} \mathrm{C}$ NMR $\left(101 \mathrm{MHz}, \mathrm{CDCl}_{3}\right) \delta 206.6,162.1(\mathrm{~d}, J=246.1 \mathrm{~Hz}), 136.4,130.6(\mathrm{~d}, J=$ $3.0 \mathrm{~Hz}), 128.6,128.4(\mathrm{~d}, J=7.8 \mathrm{~Hz}), 127.3,126.0,115.7$ (d, $J=21.7 \mathrm{~Hz}), 104.9,95.7$, 16.9 .

HR-ESI-MS m/z calcd for $\mathrm{C}_{16} \mathrm{H}_{14} \mathrm{~F}[\mathrm{M}+\mathrm{H}]^{+}: 225.1074$, found: 225.1082 .

\section{4-(4-Phenylbuta-2,3-dien-2-yl)benzo nitrile (1i)}<smiles>CC(=Cc1ccccc1)c1ccc(C#N)cc1</smiles>

Prepared by the Method A: 4-Ethynylbenzonitrile (591.9 mg, $4.7 \mathrm{mmol}, 1.0$ equiv), $n$-BuLi (2.0 mL, 2.4 M in hexane, 1.0 equiv), benzaldehyde (475 $\mu \mathrm{L}, 1.0$ equiv), $\mathrm{Ac}_{2} \mathrm{O}$ (705 $\mu \mathrm{L}, 1.6$ equiv) and THF $(10 \mathrm{~mL})$.

Step 1: The residue was purified by column chromatography on silica gel (eluent: $\mathrm{PE} / \mathrm{EtOAc}=10 / 1, \mathrm{v} / \mathrm{v}) .161 .6 \mathrm{mg}, 13 \%$ yield, a light yellow oil.

Step 2: The residue was purified by column chromatography on silica gel (eluent: $\mathrm{PE} / \mathrm{EtOAc}=30 / 1, \mathrm{v} / \mathrm{v}) .112 .6 \mathrm{mg}, 83 \%$ yield, a colorless oil.

${ }^{1} \mathrm{H}$ NMR (400 MHz, $\left.\mathrm{CDCl}_{3}\right) \delta 7.62-7.59(\mathrm{~m}, 2 \mathrm{H}), 7.55-7.52(\mathrm{~m}, 2 \mathrm{H}), 7.36-7.30$ (m, 4H), $7.27-7.23(\mathrm{~m}, 1 \mathrm{H}), 6.56(\mathrm{q}, J=2.9 \mathrm{~Hz}, 1 \mathrm{H}), 2.24(\mathrm{~d}, J=3.0 \mathrm{~Hz}, 3 \mathrm{H})$. ${ }^{13} \mathrm{C}$ NMR $\left(101 \mathrm{MHz}, \mathrm{CDCl}_{3}\right) \delta 208.2,141.6,133.5,132.4,129.0,127.7,127.2,126.5$, $119.2,110.4,104.0,97.6,16.6$.

HR-ESI-MS m/z calcd for $\mathrm{C}_{17} \mathrm{H}_{14} \mathrm{~N}[\mathrm{M}+\mathrm{H}]^{+}: 232.1121$, found: 232.1121 . 
<smiles>CC(=Cc1ccccc1)c1ccc(F)cc1</smiles>

Prepared by the Method A: 1-Ethynyl-4-fluorobenzene (575 $\mu \mathrm{L}, 5.0 \mathrm{mmol}, 1.0$ equiv), $n$-BuLi (2.0 mL, 2.5 M in hexane, 1.0 equiv), benzaldehyde (510 $\mu \mathrm{L}, 1.0$ equiv), $\mathrm{Ac}_{2} \mathrm{O}$ (755 $\mu \mathrm{L}, 1.6$ equiv) and THF $(10 \mathrm{~mL})$.

Step 1: The residue was purified by column chromatography on silica gel (eluent: $\mathrm{PE} / \mathrm{EtOAc}=30 / 1, \mathrm{v} / \mathrm{v}) .466 .6 \mathrm{mg}, 35 \%$ yield, a light yellow oil.

Step 2: The residue was purified by column chromato graphy on silica gel (eluent: PE). $240.8 \mathrm{mg}, 62 \%$ yield, a colorless oil.

${ }^{1} \mathrm{H}$ NMR (400 MHz, $\left.\mathrm{CDCl}_{3}\right) \delta 7.68-7.64(\mathrm{~m}, 2 \mathrm{H}), 7.63-7.60(\mathrm{~m}, 2 \mathrm{H}), 7.58-7.54$ (m, 2H), $7.49-7.44(\mathrm{~m}, 1 \mathrm{H}), 7.30-7.23(\mathrm{~m}, 2 \mathrm{H}), 6.75$ (q, $J=2.8 \mathrm{~Hz}, 1 \mathrm{H}), 2.47$ (d, $J$ $=2.8 \mathrm{~Hz}, 3 \mathrm{H})$.

${ }^{13} \mathrm{C} \mathrm{NMR}\left(101 \mathrm{MHz}, \mathrm{CDCl}_{3}\right) \delta 206.6(\mathrm{~d}, J=2.2 \mathrm{~Hz}), 162.1(\mathrm{~d}, J=246.4 \mathrm{~Hz}), 134.4$, $132.4(\mathrm{~d}, J=3.2 \mathrm{~Hz}), 128.8,127.5(\mathrm{~d}, J=7.9 \mathrm{~Hz}), 127.2,127.0,115.4(\mathrm{~d}, J=21.5$ Hz), 103.8, 96.9, 16.9.

HR-ESI-MS m/z calcd for $\mathrm{C}_{16} \mathrm{H}_{14} \mathrm{~F}[\mathrm{M}+\mathrm{H}]^{+}:$225.1074, found: 225.1075 .

\section{1-Fluoro-3-(4-phenylbuta-2,3-dien-2-yl)benzene (11)}<smiles>CC(=Cc1ccccc1)c1cccc(F)c1</smiles>

Prepared by the Method A: 1-Ethynyl-3-fluorobenzene (580 $\mu \mathrm{L}, 5.0 \mathrm{mmol}, 1.0$ equiv), $n$ - $\mathrm{BuLi}$ (2.0 mL, 2.5 M in hexane, 1.0 equiv), benzaldehyde (510 $\mu \mathrm{L}, 1.0$ equiv), $\mathrm{Ac}_{2} \mathrm{O}$ (755 $\mu \mathrm{L}, 1.6$ equiv) and THF (10 mL).

Step 1: The residue was purified by column chromatography on silica gel (eluent: $\mathrm{PE} / \mathrm{EtOAc}=30 / 1, \mathrm{v} / \mathrm{v}) .461 .2 \mathrm{mg}, 34 \%$ yield, a light yellow oil. 
Step 2: The residue was purified by column chromatography on silica gel (eluent: PE). $235.1 \mathrm{mg}, 62 \%$ yield, a colorless oil.

${ }^{1} \mathrm{H}$ NMR (400 MHz, CDCl $) \delta 7.46-7.39(\mathrm{~m}, 4 \mathrm{H}), 7.38-7.26(\mathrm{~m}, 4 \mathrm{H}), 7.05-6.99$ (m, 1H), $6.61(\mathrm{q}, J=3.0 \mathrm{~Hz}, 1 \mathrm{H}), 2.32(\mathrm{~d}, J=2.7 \mathrm{~Hz}, 3 \mathrm{H})$.

${ }^{13} \mathrm{C} \mathrm{NMR}\left(101 \mathrm{MHz}, \mathrm{CDCl}_{3}\right) \delta 207.1,163.3(\mathrm{~d}, J=245.0 \mathrm{~Hz}), 139.1(\mathrm{~d}, J=7.3 \mathrm{~Hz})$, 134.2, 129.9 (d, $J=8.4 \mathrm{~Hz}), 128.9,127.4,127.1,121.6$ (d, $J=2.4 \mathrm{~Hz}), 113.9$ (d, $J=$ $21.4 \mathrm{~Hz}), 112.8$ (d, $J=22.5 \mathrm{~Hz}), 104.0(\mathrm{~d}, J=2.6 \mathrm{~Hz}), 97.1,16.8$.

HR-ESI-MS m/z calcd for $\mathrm{C}_{16} \mathrm{H}_{14} \mathrm{~F}[\mathrm{M}+\mathrm{H}]^{+}: 225.1074$, found: 225.1089 .

\section{Deca-3,4-dien-3-ylbenzene (10)}<smiles>CCCCCC=C=C(CC)c1ccccc1</smiles>

Prepared by the Method B: Phenylacetylene (550 $\mu \mathrm{L}, 5 \mathrm{mmol}, 1.0$ equiv), $n$-BuLi (2.1 mL, 2.4 $\mathrm{M}$ in hexane, 1.0 equiv), $n$-hexanal (615 $\mu \mathrm{L}, 1.0$ equiv), $\mathrm{Ac}_{2} \mathrm{O}$ (755 $\mu \mathrm{L}$, 1.6 equiv) and THF (10 mL).

Step 1: The residue was purified by column chromatography on silica gel (eluent: $\mathrm{PE} / \mathrm{EtOAc}=30 / 1, \mathrm{v} / \mathrm{v}) .271 .1 \mathrm{mg}, 22 \%$ yield, a light yellow oil.

Step 2: The residue was purified by column chromatography on silica gel (eluent: PE). $68.2 \mathrm{mg}, 30 \%$ yield, a colorless oil.

${ }^{1} \mathrm{H}$ NMR (400 MHz, $\left.\mathrm{CDCl}_{3}\right) \delta 7.42(\mathrm{~d}, J=7.7 \mathrm{~Hz}, 2 \mathrm{H}), 7.33-7.29$ (m, 2H), $7.20-$ $7.16(\mathrm{~m}, 1 \mathrm{H}), 5.60-5.52(\mathrm{~m}, 1 \mathrm{H}), 2.43(\mathrm{qd}, J=7.2,3.7 \mathrm{~Hz}, 2 \mathrm{H}), 2.12(\mathrm{q}, J=7.1 \mathrm{~Hz}$, 2H), $1.50-1.45(\mathrm{~m}, 2 \mathrm{H}), 1.38-1.29(\mathrm{~s}, 4 \mathrm{H}), 1.14(\mathrm{t}, J=7.4 \mathrm{~Hz}, 3 \mathrm{H}), 0.90(\mathrm{t}, J=6.9$ $\mathrm{Hz}, 3 \mathrm{H})$.

${ }^{13} \mathrm{C}$ NMR (101 MHz, $\left.\mathrm{CDCl}_{3}\right) \delta 203.7,137.8,128.4,126.4,126.0,107.4,95.4,31.6$, 29.3, 29.2, 23.0, 22.6, 14.2, 12.8 .

HR-ESI-MS m/z calcd for $\mathrm{C}_{16} \mathrm{H}_{23}[\mathrm{M}+\mathrm{H}]^{+}: 215.1794$, found: 215.1804 .

Note: Allene substrates are unstable and should be characterized and used immediately for the reactions after preparation. 


\subsection{Synthesis of sulfur reagents}

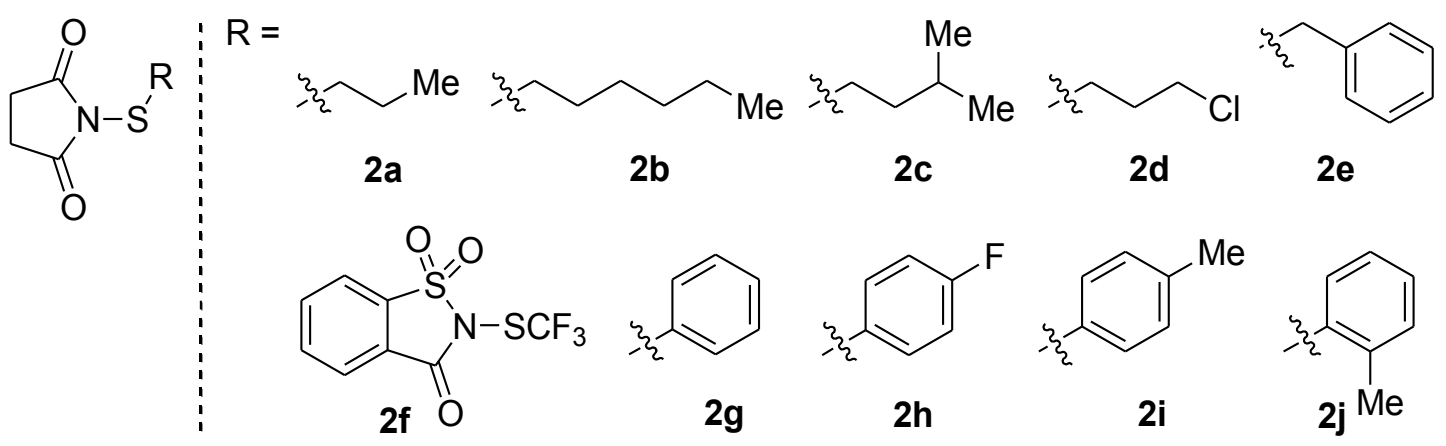

\subsubsection{Synthesis of $2 a, 2 c$ and $2 d$.}

General procedure for the synthesis of compounds $\mathbf{2 a}, \mathbf{2 c}$ and $\mathbf{2 d}$ was based on the known procedure. ${ }^{11}$ Compounds $\mathbf{2 a}, \mathbf{2 c}$ and $\mathbf{2 d}$ have been reported and their spectral data were in accordance with the reported in the literature. ${ }^{12}$

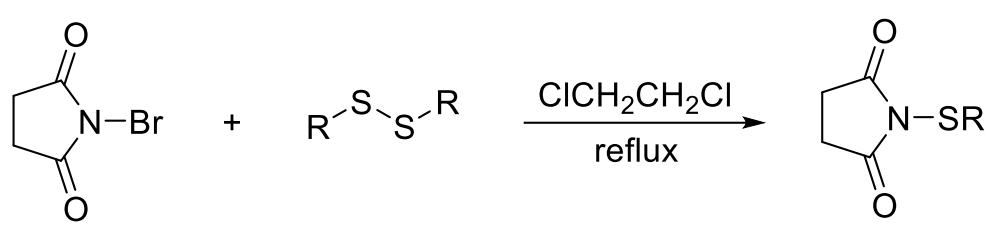

2a, 2c, 2d

To a solution of NBS $(890 \mathrm{mg}, 5.0 \mathrm{mmol})$ in $\mathrm{ClCH}_{2} \mathrm{CH}_{2} \mathrm{Cl}(13 \mathrm{~mL})$ was added disulfide (5.0 mmol, 1.0 equiv). The solution was refluxed in an oil bath for $2 \mathrm{~h}$, and then cooled to room temperature and concentrated under reduced pressure. The residue was purified by flash silica gel column chromatography to give the desired product.

\subsubsection{Synthesis of $2 b, 2 e, 2 g-2 j$.}

General procedure for the synthesis of compounds $\mathbf{2 b}, \mathbf{2 e}, \mathbf{2 g}-\mathbf{2} \mathbf{j}$ was based on the known procedure. ${ }^{13}$ Compounds $\mathbf{2 b},{ }^{12} \mathbf{2 e},{ }^{12} \mathbf{2 g},{ }^{14} \mathbf{2 h},{ }^{15} \mathbf{2} \mathbf{i},{ }^{14}$ and $\mathbf{2} \mathbf{g}^{15}$ are known and their spectral data are in accordance with the reported in the literature. 


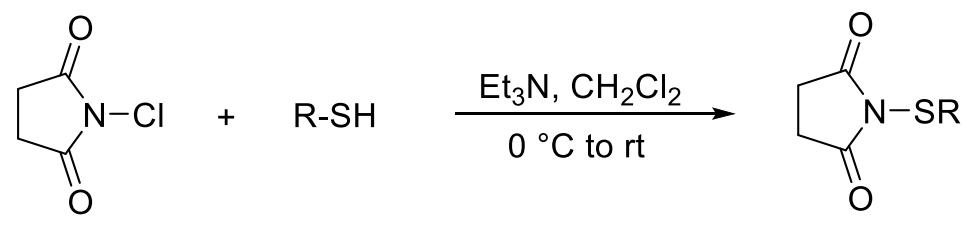

$2 b, 2 e-2 i$

To a solution of $\mathrm{NCS}(267 \mathrm{mg}, 2.0 \mathrm{mmol})$ in $\mathrm{CH}_{2} \mathrm{Cl}_{2}(10 \mathrm{~mL})$ was added dropwise thiol or thiophenol (2.0 mmol, 1.0 equiv) under $\mathrm{N}_{2}$ atmosphere at $0{ }^{\circ} \mathrm{C}$. After stirring for $5 \mathrm{~min}$, a solution of $\mathrm{Et}_{3} \mathrm{~N}(420 \mu \mathrm{L}, 3.0 \mathrm{mmol})$ in $\mathrm{CH}_{2} \mathrm{Cl}_{2}(5 \mathrm{~mL})$ was added dropwise over $10 \mathrm{~min}$. The resultant solution was allowed to warm up to room temperature and stirred for $2 \mathrm{~h}$. Water $(10 \mathrm{~mL})$ was added. Then, the mixture was extracted with $\mathrm{CH}_{2} \mathrm{Cl}_{2}(15 \mathrm{~mL} \times 3)$. The combined organic layers were dried over anhydrous $\mathrm{Na}_{2} \mathrm{SO}_{4}$, filtered and concentrated under reduced pressure. The residue was purified by flash silica gel column chromatography to give the desired product.

\subsubsection{Synthesis of $2 f$.}

Compound $\mathbf{2 f}$ was prepared according to the literature method. ${ }^{16}$

\section{General procedure for the catalytic reactions}

\subsection{Intramolecular thiocarbocyclization}

\subsubsection{General procedure for PhSePh-catalyzed intramolecular thiocarbocyclization} of allenes

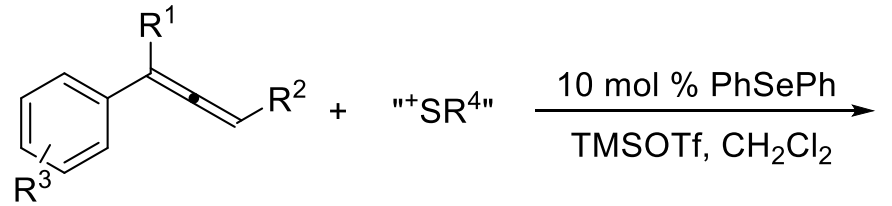<smiles>[R]SC1=C([R])c2cc[R]([R])cc2C1[R]</smiles>

Method D: To a $10 \mathrm{~mL}$ Schlenk flask charged with a magnetic stirring bar were added allene ( $0.1 \mathrm{mmol}, 1.0$ equiv), sulfur reagent ( $0.1 \mathrm{mmol}, 1.0$ equiv), selenide ( 2.4 $\mathrm{mg}, 10 \mathrm{~mol} \%)$ and $\mathrm{CH}_{2} \mathrm{Cl}_{2}(2 \mathrm{~mL})$ subsequently. The flask was placed into a cooling bath at $-60^{\circ} \mathrm{C}$. TMSOTf $(18.4 \mu \mathrm{L}, 1.0$ equiv $)$ was added after the solution was stirred for about $5 \mathrm{~min}$ at $-60{ }^{\circ} \mathrm{C}$. The resulting solution was stirred at this temperature for 22 
$\mathrm{h}$, then quenched with triethylamine, and concentrated under reduced pressure. The residue was purified by preparative thin layer chromatography to afford the product.

Note: The synthesized target product is unstable and will decompose after a long time.

\subsubsection{Kinetic resolution reaction}<smiles>COc1ccc(C=C=C(C)c2ccccc2)cc1</smiles>

$1 f$<smiles>O=C1C[CH+]CC1=O</smiles>

2i

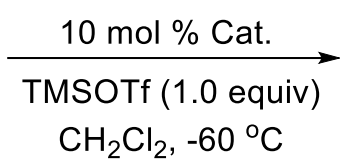

$\mathrm{CH}_{2} \mathrm{Cl}_{2},-60^{\circ} \mathrm{C}$<smiles></smiles>

3aa

Catalysts C5, C6 and $\mathbf{C 7}$ were synthesized according to the literature methods. ${ }^{17}$<smiles>CCCC(C)(C)c1cc([18O]c2cc(C(C)(C)C)cc(C(C)(C)C)c2OC)c2ccccc2c1</smiles>

C5<smiles>CCN[C@@H]1c2ccccc2CC1[Se]c1c(C)cccc1OC</smiles>

C6<smiles>CCC[C@H]1c2ccccc2CC1Sc1c(C)cccc1OC</smiles>

C7

\begin{tabular}{ccccc}
\hline entry & catalyst & time $(\mathrm{h})$ & yield of 3aa $(\%)^{a}$ & ee of 3aa $(\%)^{b}$ \\
\hline 1 & C5 & 22 & 61 & 0 \\
2 & C6 & 22 & 61 & 0 \\
3 & C7 & 22 & 27 & 0 \\
4 & C5 & 1 & 40 & 0 \\
5 & C6 & 1 & 40 & 0 \\
\hline
\end{tabular}

Reaction conditions: $\mathbf{1 f}(0.05 \mathrm{mmol}), \mathbf{2 i}(0.05 \mathrm{mmol})$, catalyst (10 mol \%), TMSOTf (0.05 mmol), $\quad \mathrm{CH}_{2} \mathrm{Cl}_{2} \quad\left(\begin{array}{ll}1.0 & \mathrm{~mL}\end{array}\right), \quad-60 \quad{ }^{\circ} \mathrm{C}$. ${ }^{a}$ Refer to NMR yield using 1,3,5-trimethoxybenzene as the internal standard. ${ }^{b}$ The ee values were determined by HPLC analysis.

\subsection{Intermolecular azidothiolation}

\subsubsection{Condition optimization ${ }^{a}$}




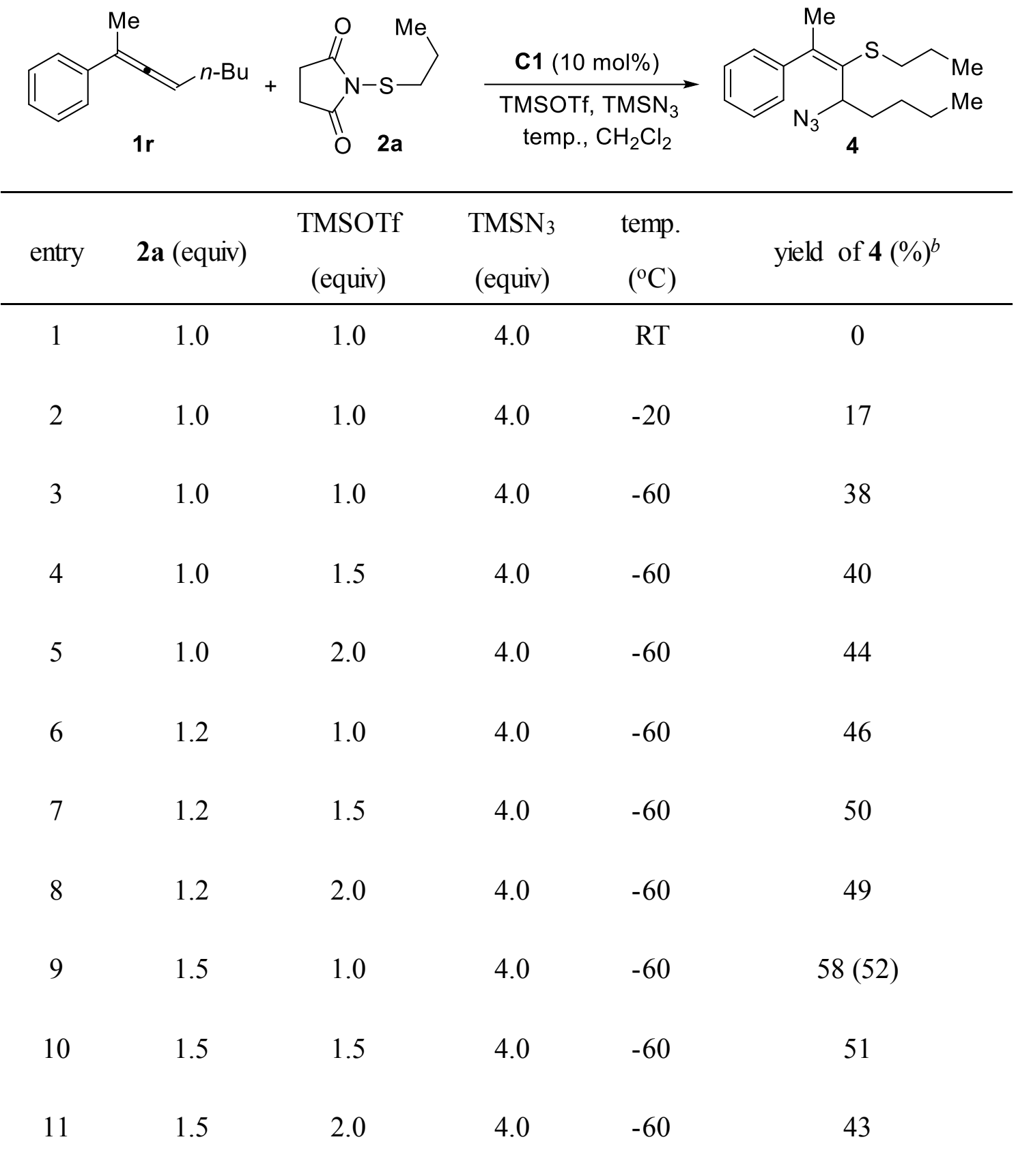

${ }^{a}$ Reaction conditions: $1 \mathbf{r}(0.05 \mathrm{mmol}), \mathbf{C 1}(10 \mathrm{~mol} \%), \mathrm{TMSN}_{3}$ (4.0 equiv), $\mathrm{CH}_{2} \mathrm{Cl}_{2}$ (1.0 mL), 22 h. ${ }^{b}$ Refer to NMR yield using 1,3,5-trimethoxybenzene as the internal standard; isolated yield is in parenthesis in $0.1 \mathrm{mmol}$ scale.

\subsubsection{Synthetic procedure}




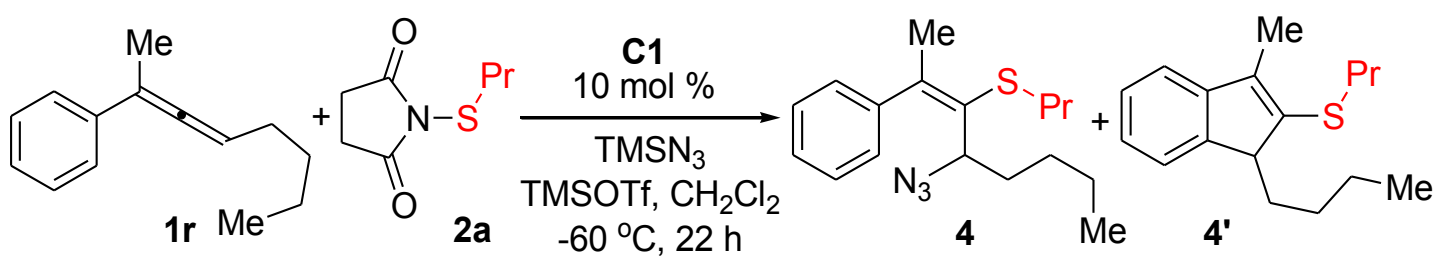

Method E: To a 10-mL Schlenk flask charged with a magnetic stirring bar were added allene $1 \mathbf{r}(0.1 \mathrm{mmol}, 1.0$ equiv), 2a ( $0.15 \mathrm{mmol}, 1.5$ equiv), $\mathrm{PhSePh}(2.4 \mathrm{mg}$, $10 \mathrm{~mol} \%$ ), and $\mathrm{CH}_{2} \mathrm{Cl}_{2}(2 \mathrm{~mL})$ subsequently. The flask was placed into a cooling bath at $-60{ }^{\circ} \mathrm{C} . \mathrm{TMSN}_{3}(52.2 \mu \mathrm{L}, 4.0$ equiv) was added. TMSOTf $(18.4 \mu \mathrm{L}, 1.0$ equiv) was added to the solution after about $5 \mathrm{~min}$. The resulting solution was stirred at $-60^{\circ} \mathrm{C}$ for $22 \mathrm{~h}$, then quenched with triethylamine and concentrated under reduced pressure. The mixture was purified by preparative thin layer chromatography (eluent: PE) to afford the products.

\subsection{The procedure for the synthesis of 3 a in gram scale}

To a $250 \mathrm{~mL}$ Schlenk flask charged with a magnetic stirring bar were added 1a (5.0 mmol, $1030.5 \mathrm{mg})$, sulfur reagent 2 a $(5.0 \mathrm{mmol}, 865.3 \mathrm{mg})$, selenide $(117.0 \mathrm{mg}, 10$ mol \%) and $\mathrm{CH}_{2} \mathrm{Cl}_{2}(100 \mathrm{~mL})$ subsequently. The flask was placed into a cooling bath at $-60^{\circ} \mathrm{C}$. TMSOTf $(920.2 \mu \mathrm{L}, 1.0$ equiv) was added after the solution was stirred for about $5 \mathrm{~min}$ at $-60{ }^{\circ} \mathrm{C}$. The resulting solution was stirred at this temperature for $22 \mathrm{~h}$, then quenched with triethylamine, and concentrated under reduced pressure. The residue was purified by silica gel collumn chromatography (eluent: PE) to afford the product 3a (1148.5 $\mathrm{mg}, 82 \%$ yield).

\section{Characterization data}

\section{(3-Methyl-1-phenyl-1H-inden-2-yl)(propyl)sulfane (3a)}<smiles>CCCSC1=C(C)c2ccccc2C1c1ccccc1</smiles> 
Prepared by the method D. Purified by preparative thin layer chromatography (eluent:

PE) to afford $\mathbf{3 a}(23.9 \mathrm{mg}, 85 \%$ yield $)$ as a colorless oil.

${ }^{1} \mathrm{H}$ NMR $\left(400 \mathrm{MHz}, \mathrm{CDCl}_{3}\right) \delta 7.32-7.27(\mathrm{~m}, 4 \mathrm{H}), 7.26-7.22(\mathrm{~m}, 1 \mathrm{H}), 7.16-7.08$ (m, 4H), $4.60(\mathrm{q}, J=2.1 \mathrm{~Hz}, 1 \mathrm{H}), 2.61(\mathrm{ddd}, J=12.7,8.1,6.0 \mathrm{~Hz}, 1 \mathrm{H}), 2.39$ (ddd, $J=$ 12.7, 8.1, 6.9 Hz, 1H), 2.29 (d, $J=2.0 \mathrm{~Hz}, 3 \mathrm{H}), 1.55-1.45(\mathrm{~m}, 2 \mathrm{H}), 0.90$ (t, $J=7.3$ $\mathrm{Hz}, 3 \mathrm{H})$.

${ }^{13} \mathrm{C}$ NMR $\left(101 \mathrm{MHz}, \mathrm{CDCl}_{3}\right) \delta 148.4,144.9,141.4,139.8,139.3,128.8,128.4$, $127.04,127.00,125.4,123.6,118.7,58.7,35.7,23.5,13.3,11.8$.

HR-ESI-MS m/z calcd for $\mathrm{C}_{19} \mathrm{H}_{21} \mathrm{~S}[\mathrm{M}+\mathrm{H}]^{+}: 281.1358$, found: 281.1367 .

\section{Hexyl(3-methyl-1-phenyl-1 H-inden-2-yl)sulfane (3b)}

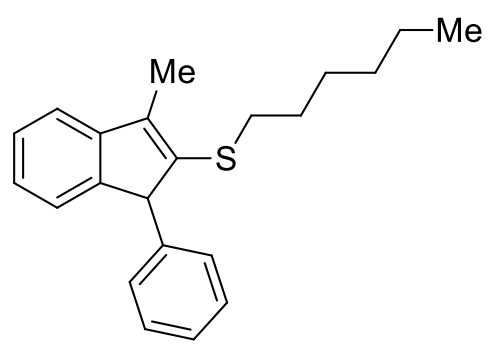

Prepared by the method D. Purified by preparative thin layer chromatography (eluent: PE) to afford $\mathbf{3 b}(26.9 \mathrm{mg}, 83 \%$ yield) as a colorless oil.

${ }^{1} \mathrm{H}$ NMR (400 MHz, $\left.\mathrm{CDCl}_{3}\right) \delta 7.31$ - 7.27 (m, 4H), 7.25 - $7.21(\mathrm{~m}, 1 \mathrm{H}), 7.15-7.07$ $(\mathrm{m}, 4 \mathrm{H}), 4.59(\mathrm{q}, J=2.1 \mathrm{~Hz}, 1 \mathrm{H}), 2.60(\mathrm{ddd}, J=12.6,8.4,6.0 \mathrm{~Hz}, 1 \mathrm{H}), 2.41(\mathrm{ddd}, J=$ 12.6, 8.4, $6.8 \mathrm{~Hz}, 1 \mathrm{H}), 2.27(\mathrm{~d}, J=2.0 \mathrm{~Hz}, 3 \mathrm{H}), 1.48-1.40(\mathrm{~m}, 2 \mathrm{H}), 1.28-1.18(\mathrm{~m}$, $6 \mathrm{H}), 0.86(\mathrm{t}, J=7.1 \mathrm{~Hz}, 3 \mathrm{H})$.

${ }^{13} \mathrm{C} \mathrm{NMR}\left(101 \mathrm{MHz}, \mathrm{CDCl}_{3}\right) \delta 148.4,145.0,141.3,139.8,139.4,128.8,128.4,127.1$, $127.0,125.4,123.6,118.7,58.7,33.7,31.5,30.2,28.4,22.7,14.1,11.8$.

HR-ESI-MS m/z calcd for $\mathrm{C}_{22} \mathrm{H}_{27} \mathrm{~S}[\mathrm{M}+\mathrm{H}]^{+}: 323.1828$, found: 323.1838 .

\section{Isopentyl(3-methyl-1-phenyl-1 $H$-inden-2-yl)sulfane (3c)}


<smiles>CC1=C(SCCC(C)C)C(c2ccccc2)c2ccccc21</smiles>

Prepared by the method D. Purified by preparative thin layer chromatography (eluent: PE) to afford $\mathbf{3 c}(24.2 \mathrm{mg}, 79 \%$ yield $)$ as a colorless oil.

${ }^{1} \mathrm{H}$ NMR $\left(400 \mathrm{MHz}, \mathrm{CDCl}_{3}\right) \delta 7.32-7.28(\mathrm{~m}, 4 \mathrm{H}), 7.26-7.22(\mathrm{~m}, 1 \mathrm{H}), 7.16-7.09$ $(\mathrm{m}, 4 \mathrm{H}), 4.60(\mathrm{~s}, 1 \mathrm{H}), 2.64-2.57(\mathrm{~m}, 1 \mathrm{H}), 2.47-2.40(\mathrm{~m}, 1 \mathrm{H}), 2.28(\mathrm{~d}, J=1.9 \mathrm{~Hz}$, $3 \mathrm{H}), 1.62-1.55(\mathrm{~m}, 1 \mathrm{H}), 1.39-1.33(\mathrm{~m}, 2 \mathrm{H}), 0.82(\mathrm{~d}, J=6.6 \mathrm{~Hz}, 6 \mathrm{H})$.

${ }^{13} \mathrm{C}$ NMR (101 MHz, $\left.\mathrm{CDCl}_{3}\right) \delta 148.4,145.0,141.1,139.8,139.4,128.8,128.4,127.1$, $127.0,125.3,123.6,118.7,58.7,39.2,31.7,27.5,22.5,22.2,11.8$.

HR-ESI-MS m/z calcd for $\mathrm{C}_{21} \mathrm{H}_{25} \mathrm{~S}[\mathrm{M}+\mathrm{H}]^{+}$: 309.1671, found: 309.1681 .

\section{(3-Chloropropyl)(3-methyl-1-phenyl-1 H-inden-2-yl)sulfane (3d)}<smiles>CC1=C(SCCCCl)C(c2ccccc2)c2ccccc21</smiles>

Prepared by the method D. Purified by preparative thin layer chromatography (eluent: PE) to afford $\mathbf{3 d}(23.5 \mathrm{mg}, 75 \%$ yield) as a colorless oil.

${ }^{1} \mathrm{H}$ NMR (400 MHz, $\left.\mathrm{CDCl}_{3}\right) \delta 7.33-7.27$ (m, 5H), 7.18 - $7.15(\mathrm{~m}, 2 \mathrm{H}), 7.12-7.09$ (m, 2H), 4.62 (q, $J=2.1 \mathrm{~Hz}, 1 \mathrm{H}), 3.53(\mathrm{t}, J=6.3 \mathrm{~Hz}, 2 \mathrm{H}), 2.77$ (ddd, $J=13.4,7.4$, $6.1 \mathrm{~Hz}, 1 \mathrm{H}), 2.54(\mathrm{dt}, J=13.3,7.2 \mathrm{~Hz}, 1 \mathrm{H}), 2.30(\mathrm{~d}, J=2.0 \mathrm{~Hz}, 3 \mathrm{H}), 1.94-1.86(\mathrm{~m}$, $2 \mathrm{H})$.

${ }^{13} \mathrm{C}$ NMR $\left(101 \mathrm{MHz}, \mathrm{CDCl}_{3}\right) \delta 148.4,144.7,142.7,139.6,138.2,128.8,128.5,127.2$, $127.1,125.8,123.7,118.9,58.7,43.4,32.8,30.7,11.9$.

HR-ESI-MS m/z calcd for $\mathrm{C}_{19} \mathrm{H}_{20} \mathrm{SCl}[\mathrm{M}+\mathrm{H}]^{+}: 315.0969$, found: 315.0974 . 
<smiles>CC1=C(SCc2ccccc2)C(c2ccccc2)c2ccccc21</smiles>

Prepared by the method D. Purified by preparative thin layer chromatography (eluent: PE) to afford $\mathbf{3 e}(27.2 \mathrm{mg}, 83 \%$ yield) as a colorless oil.

${ }^{1} \mathrm{H}$ NMR (400 MHz, $\left.\mathrm{CDCl}_{3}\right) \delta 7.32-7.27$ (m, 4H), 7.25 - $7.19(\mathrm{~m}, 4 \mathrm{H}), 7.14-7.11$ (m, 4H), $7.09-7.05(\mathrm{~m}, 2 \mathrm{H}), 4.48$ (q, $J=2.1 \mathrm{~Hz}, 1 \mathrm{H}), 3.70(\mathrm{~d}, J=13.0 \mathrm{~Hz}, 1 \mathrm{H}), 3.58$ $(\mathrm{d}, J=13.0 \mathrm{~Hz}, 1 \mathrm{H}), 2.10(\mathrm{~d}, J=2.1 \mathrm{~Hz}, 3 \mathrm{H})$.

${ }^{13} \mathrm{C}$ NMR (101 MHz, $\left.\mathrm{CDCl}_{3}\right) \delta 148.4,144.8,143.1,139.5,138.43,138.36,128.9$, $128.8,128.6,128.5,127.2,127.1,127.0,125.7,123.7,119.1,59.0,38.6,11.7$.

HR-ESI-MS m/z calcd for $\mathrm{C}_{23} \mathrm{H}_{21} \mathrm{~S}[\mathrm{M}+\mathrm{H}]^{+}: 329.1358$, found: 329.1356 .

(3-Methyl-1-phenyl-1H-inden-2-yl)(trifluoromethyl)s ulfane (3f)<smiles>CC1=C(C)c2ccccc2C1=C(C)C(F)(F)F</smiles>

Prepared by the method D. Purified by preparative thin layer chromatography (eluent: PE) to afford $\mathbf{3 f}(24.3 \mathrm{mg}, 79 \%$ yield) as a colorless oil. ${ }^{1} \mathrm{H}$ NMR (400 MHz, $\left.\mathrm{CDCl}_{3}\right) \delta 7.51-7.49(\mathrm{~m}, 2 \mathrm{H}), 7.42-7.38(\mathrm{~m}, 1 \mathrm{H}), 7.31-7.28$ (m, 4H), 7.02 (dd, $J=7.4,2.0 \mathrm{~Hz}, 2 \mathrm{H}), 4.73$ (q, $J=2.4 \mathrm{~Hz}, 1 \mathrm{H}), 2.44$ (d, $J=2.3 \mathrm{~Hz}$, $3 \mathrm{H})$.

${ }^{13} \mathrm{C}$ NMR $\left(101 \mathrm{MHz}, \mathrm{CDCl}_{3}\right) \delta 153.0,149.4,143.4,138.1,133.1,129.7$ (q, $J=311.5$ Hz), 129.5, 128.9, 128.6, 127.8, 127.4, 127.3, 124.3, 120.7, 60.0, 12.4.

${ }^{19} \mathrm{~F}$ NMR $\left(377 \mathrm{MHz}, \mathrm{CDCl}_{3}\right) \delta-41.16$.

HR-ESI-MS m/z calcd for $\mathrm{C}_{17} \mathrm{H}_{14} \mathrm{~F}_{3} \mathrm{~S}[\mathrm{M}+\mathrm{H}]^{+}: 307.0763$, found: 307.0775 . 
<smiles>CC1=C(Sc2ccccc2)C(c2ccccc2)c2ccccc21</smiles>

Prepared by the method D. Purified by preparative thin layer chromatography (eluent: PE) to afford $\mathbf{3 g}$ (26.1 $\mathrm{mg}, 83 \%$ yield) as a colorless oil.

${ }^{1} \mathrm{H}$ NMR (400 MHz, $\left.\mathrm{CDCl}_{3}\right) \delta 7.40-7.38(\mathrm{~m}, 1 \mathrm{H}), 7.36-7.32(\mathrm{~m}, 1 \mathrm{H}), 7.23-7.13$ (m, 10H), $6.87-6.84(\mathrm{~m}, 2 \mathrm{H}), 4.56$ (q, $J=2.2 \mathrm{~Hz}, 1 \mathrm{H}), 2.33$ (d, $J=2.1 \mathrm{~Hz}, 3 \mathrm{H})$.

${ }^{13} \mathrm{C}$ NMR $\left(101 \mathrm{MHz}, \mathrm{CDCl}_{3}\right) \delta 148.5,144.7,139.3,137.9,136.4,129.8,129.0$, $128.60,128.55,127.1,127.0,126.28,126.25,124.0,119.4,58.2,12.1$.

HR-ESI-MS m/z calcd for $\mathrm{C}_{22} \mathrm{H}_{19} \mathrm{~S}[\mathrm{M}+\mathrm{H}]^{+}: 315.1202$, found: 315.1205 .

\section{(4-Fluorophenyl)(3-methyl-1-phenyl-1H-inden-2-yl)sulfane (3h)}

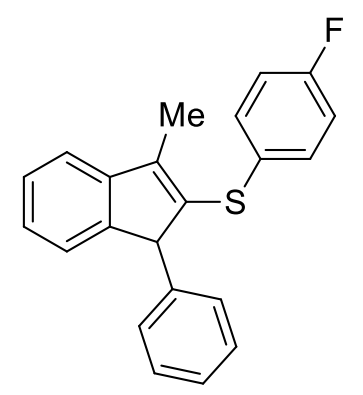

Prepared by the method D. Purified by preparative thin layer chromatography (eluent: PE) to afford $\mathbf{3 h}(24.2 \mathrm{mg}, 73 \%$ yield) as a colorless oil.

${ }^{1} \mathrm{H}$ NMR $\left(400 \mathrm{MHz}, \mathrm{CDCl}_{3}\right) \delta$ 7.37- $7.30(\mathrm{~m}, 2 \mathrm{H}), 7.21-7.10(\mathrm{~m}, 7 \mathrm{H}), 6.92-6.86$ (m, 2H), $6.85-6.80(\mathrm{~m}, 2 \mathrm{H}), 4.47$ (q, $J=2.2 \mathrm{~Hz}, 1 \mathrm{H}), 2.32$ (d, $J=2.0 \mathrm{~Hz}, 3 \mathrm{H})$. ${ }^{13} \mathrm{C} \mathrm{NMR}\left(101 \mathrm{MHz}, \mathrm{CDCl}_{3}\right) \delta 162.0(\mathrm{~d}, J=246.6 \mathrm{~Hz}), 148.4,144.6,143.6,139.2$, 138.4, 132.6 (d, $J=8.1 \mathrm{~Hz}), 130.9$ (d, $J=3.0 \mathrm{~Hz}), 127.2,127.0,126.2,123.9,119.3$, $116.0(\mathrm{~d}, J=21.9 \mathrm{~Hz}), 58.2,12.0$.

HR-ESI-MS m/z calcd for $\mathrm{C}_{22} \mathrm{H}_{18} \mathrm{SF}[\mathrm{M}+\mathrm{H}]^{+}: 333.1108$, found: 333.1105 . 


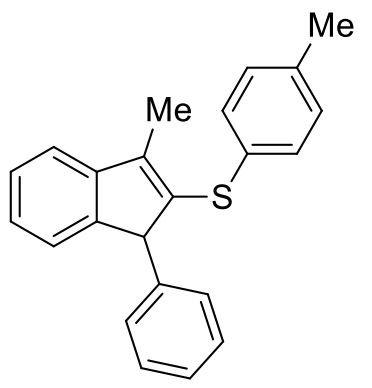

Prepared by the method D. Purified by preparative thin layer chromatography (eluent: PE) to afford $3 \mathbf{i}(25.9 \mathrm{mg}, 79 \%$ yield) as a colorless oil.

${ }^{1} \mathrm{H}$ NMR (400 MHz, CDCl 3 ) $\delta 7.37-7.29(\mathrm{~m}, 2 \mathrm{H}), 7.21-7.17(\mathrm{~m}, 3 \mathrm{H}), 7.15-7.10$ $(\mathrm{m}, 2 \mathrm{H}), 7.07-7.04(\mathrm{~m}, 2 \mathrm{H}), 7.02-7.00(\mathrm{~m}, 2 \mathrm{H}), 6.86-6.82(\mathrm{~m}, 2 \mathrm{H}), 4.51(\mathrm{q}, J=$ $2.1 \mathrm{~Hz}, 1 \mathrm{H}), 2.31-2.30(\mathrm{~m}, 6 \mathrm{H})$.

${ }^{13} \mathrm{C} \mathrm{NMR}\left(101 \mathrm{MHz}, \mathrm{CDCl}_{3}\right) \delta 148.5,144.8,143.5,139.4,138.8,136.5,132.5,130.5$, $129.7,128.6,128.5,127.1,126.9,126.0,124.0,120.0,58.1,21.2,12.0$.

HR-ESI-MS m/z calcd for $\mathrm{C}_{23} \mathrm{H}_{21} \mathrm{~S}[\mathrm{M}+\mathrm{H}]^{+}: 329.1358$, found: 329.1367 .

\section{(3-Methyl-1-phenyl-1H-inden-2-yl)(o-tolyl)sulfane (3j)}<smiles>CC1=C(Sc2ccccc2C)C(c2ccccc2)c2ccccc21</smiles>

Prepared by the method D. Purified by preparative thin layer chromatography (eluent: PE) to afford $\mathbf{3 j}$ ( $21.9 \mathrm{mg}, 67 \%$ yield) as a colorless oil.

${ }^{1} \mathrm{H}$ NMR (400 MHz, $\left.\mathrm{CDCl}_{3}\right) \delta 7.39-7.32(\mathrm{~m}, 2 \mathrm{H}), 7.22-7.18(\mathrm{~m}, 3 \mathrm{H}), 7.17-7.03$ (m, 6H), $6.83-6.79$ (m, 2H), 4.47 (q, $J=2.1 \mathrm{~Hz}, 1 \mathrm{H}), 2.34(\mathrm{~d}, J=2.1 \mathrm{~Hz}, 3 \mathrm{H}), 2.20$ (s, 3H).

${ }^{13} \mathrm{C} \mathrm{NMR}\left(101 \mathrm{MHz}, \mathrm{CDCl}_{3}\right) \delta 148.5,144.8,143.1,139.3,138.8,138.7,134.9,131.2$, $130.3,128.6,128.5,127.1,126.9,126.8,126.4,125.9,123.9,119.1,58.1,20.5,11.9$. 
HR-ESI-MS m/z calcd for $\mathrm{C}_{23} \mathrm{H}_{21} \mathrm{~S}[\mathrm{M}+\mathrm{H}]^{+}: 329.1358$, found: 329.1359 .

(3-Methyl-1-(p-tolyl)-1H-inden-2-yl)(propyl)sulfane (3k)<smiles>CCCSC1=C(C)c2ccccc2C1c1ccc(C)cc1</smiles>

Prepared by the method D. Purified by preparative thin layer chromatography (eluent: PE) to afford $3 \mathbf{k}(21.1 \mathrm{mg}, 72 \%$ yield ) as a colorless oil.

${ }^{1} \mathrm{H}$ NMR (400 MHz, $\left.\mathrm{CDCl}_{3}\right) \delta 7.30-7.27(\mathrm{~m}, 2 \mathrm{H}), 7.15-7.09(\mathrm{~m}, 4 \mathrm{H}), 6.99-6.97$ (m, 2H), 4.57 (q, $J=2.1 \mathrm{~Hz}, 1 \mathrm{H}), 2.63(\mathrm{ddd}, J=12.7,8.1,5.9 \mathrm{~Hz}, 1 \mathrm{H}), 2.44-2.37$ (m, 1H), $2.33(\mathrm{~s}, 3 \mathrm{H}), 2.27(\mathrm{~d}, J=2.0 \mathrm{~Hz}, 3 \mathrm{H}), 1.55-1.44(\mathrm{~m}, 2 \mathrm{H}), 0.91(\mathrm{t}, J=7.4$ $\mathrm{Hz}, 3 \mathrm{H})$.

${ }^{13} \mathrm{C} \mathrm{NMR}\left(101 \mathrm{MHz}, \mathrm{CDCl}_{3}\right) \delta 148.6,144.9,141.2,139.5,136.7,136.6,129.5,128.2$, $126.9,125.4,123.5,118.7,58.2,35.7,23.5,21.3,13.4,11.8$.

HR-ESI-MS m/z calcd for $\mathrm{C}_{20} \mathrm{H}_{23} \mathrm{~S}[\mathrm{M}+\mathrm{H}]^{+}:$295.1515, found: 295.1514 .

(1-(4-Bromophenyl)-3-methyl-1 H-inden-2-yl)(propyl)sulfane (3I)<smiles>CCCSC1=C(C)c2ccccc2C1c1ccc(Br)cc1</smiles>

Prepared by the method D. Purified by preparative thin layer chromatography (eluent: PE) to afford $\mathbf{3 1}(29.7 \mathrm{mg}, 83 \%$ yield) as a colorless oil.

${ }^{1} \mathrm{H}$ NMR (400 MHz, $\left.\mathrm{CDCl}_{3}\right) \delta 7.40(\mathrm{~d}, J=8.2 \mathrm{~Hz}, 2 \mathrm{H}), 7.30-7.29(\mathrm{~m}, 2 \mathrm{H}), 7.15-$ $7.10(\mathrm{~m}, 2 \mathrm{H}), 6.96(\mathrm{~d}, J=8.2 \mathrm{~Hz}, 2 \mathrm{H}), 4.55(\mathrm{~s}, 1 \mathrm{H}), 2.63-2.57(\mathrm{~m}, 1 \mathrm{H}), 2.44-2.37$ (m, 1H), $2.27(\mathrm{~d}, J=2.1 \mathrm{~Hz}, 3 \mathrm{H}), 1.53-1.42(\mathrm{~m}, 2 \mathrm{H}), 0.91(\mathrm{t}, J=7.3 \mathrm{~Hz}, 3 \mathrm{H})$. 
${ }^{13} \mathrm{C} \mathrm{NMR}\left(101 \mathrm{MHz}, \mathrm{CDCl}_{3}\right) \delta 147.8,144.9,142.0,139.0,138.9,131.9,130.2,127.2$, $125.6,123.5,120.8,118.9,57.9,35.9,23.5,13.3,11.9$.

HR-ESI-MS m/z calcd for $\mathrm{C}_{19} \mathrm{H}_{20} \mathrm{SBr}[\mathrm{M}+\mathrm{H}]^{+}: 359.0464$, found: 359.0471 .

\section{(1-(4-Chlorophenyl)-3-methyl-1H-inden-2-yl)(propyl)sulfane (3m)}<smiles>CCCSC1=C(C)c2ccccc2C1c1ccc(Cl)cc1</smiles>

Prepared by the method D. Purified by preparative thin layer chromatography (eluent: PE) to afford $\mathbf{3 m}(24.8 \mathrm{mg}, 79 \%$ yield) as a colorless oil.

${ }^{1} \mathrm{H} \mathrm{NMR}\left(400 \mathrm{MHz}, \mathrm{CDCl}_{3}\right) \delta 7.29(\mathrm{dd}, J=4.1,1.7 \mathrm{~Hz}, 2 \mathrm{H}), 7.25(\mathrm{dd}, J=6.8,1.7 \mathrm{~Hz}$, 2H), $7.14-7.09(\mathrm{~m}, 2 \mathrm{H}), 7.03-7.00(\mathrm{~m}, 2 \mathrm{H}), 4.56(\mathrm{q}, J=2.1 \mathrm{~Hz}, 1 \mathrm{H}), 2.60(\mathrm{dt}, J=$ 14.0, 7.4 Hz, 1 H), 2.40 (dt, $J=12.7,7.5 \mathrm{~Hz}, 1 \mathrm{H}), 2.27$ (d, $J=2.0 \mathrm{~Hz}, 3 \mathrm{H}), 1.50$ (dq, $J$ $=14.6,7.4 \mathrm{~Hz}, 2 \mathrm{H}), 0.91(\mathrm{t}, J=7.3 \mathrm{~Hz}, 3 \mathrm{H})$.

${ }^{13} \mathrm{C} \mathrm{NMR}\left(101 \mathrm{MHz}, \mathrm{CDCl}_{3}\right) \delta 147.9,144.9,142.0,138.9,138.4,132.8,129.8,129.0$, $127.2,125.6,123.5,118.9,57.8,35.9,23.5,13.3,11.9$.

HR-ESI-MS m/z calcd for $\mathrm{C}_{19} \mathrm{H}_{20} \mathrm{SCl}[\mathrm{M}+\mathrm{H}]^{+}: 315.0969$, found: 315.0971 .

\section{(1-(4-Fluorophenyl)-3-methyl-1 H-inden-2-yl)(propyl)sulfane (3n)}<smiles>CCCSC1=C(C)c2ccccc2C1c1ccc(F)cc1</smiles>

Prepared by the method D. Purified by preparative thin layer chromatography (eluent: PE) to afford $3 \mathbf{n}(24.3 \mathrm{mg}, 82 \%$ yield $)$ as a colorless oil. 
${ }^{1} \mathrm{H}$ NMR (400 MHz, $\left.\mathrm{CDCl}_{3}\right) \delta 7.30-7.29(\mathrm{~m}, 2 \mathrm{H}), 7.13-7.10(\mathrm{~m}, 2 \mathrm{H}), 7.06-7.02$ $(\mathrm{m}, 2 \mathrm{H}), 7.00-6.95(\mathrm{~m}, 2 \mathrm{H}), 4.57$ (q, $J=2.1 \mathrm{~Hz}, 1 \mathrm{H}), 2.59(\mathrm{ddd}, J=12.6,8.1,6.0$ Hz, 1H), 2.39 (ddd, $J=12.6,8.1,6.9 \mathrm{~Hz}, 1 \mathrm{H}), 2.27$ (d, $J=2.0 \mathrm{~Hz}, 3 \mathrm{H}), 1.52-1.44$ (m, 2H), 0.90 (t, $J=7.4 \mathrm{~Hz}, 3 \mathrm{H})$.

${ }^{13} \mathrm{C}$ NMR (101 MHz, $\left.\mathrm{CDCl}_{3}\right) \delta 162.0(\mathrm{~d}, J=244.8 \mathrm{~Hz}), 148.2,144.8,141.7,139.2$, $135.5(\mathrm{~d}, J=3.2 \mathrm{~Hz}), 129.9(\mathrm{~d}, J=8.0 \mathrm{~Hz}), 127.2,125.5,123.5,118.8,115.6(\mathrm{~d}, J=$ $21.3 \mathrm{~Hz}), 57.8,35.8,23.5,13.3,11.9$.

HR-ESI-MS m/z calcd for $\mathrm{C}_{19} \mathrm{H}_{20} \mathrm{FS}[\mathrm{M}+\mathrm{H}]^{+}:$299.1264, found: 299.1278 .

\section{(1-(4-Methoxyphenyl)-3-methyl-1H-inden-2-yl)(propyl)sulfane (3o)}<smiles>CCCSC1=C(C)c2ccccc2C1c1ccc(OC)cc1</smiles>

Prepared by the method D. Purified by preparative thin layer chromatography (eluent: $\left.\mathrm{PE} / \mathrm{CH}_{2} \mathrm{Cl}_{2}=4 / 1, \mathrm{v} / \mathrm{v}\right)$ to afford $\mathbf{3 o}(19.9 \mathrm{mg}, 64 \%$ yield) as a colorless oil.

${ }^{1} \mathrm{H}$ NMR (400 MHz, $\left.\mathrm{CDCl}_{3}\right) \delta 7.27$ - $7.26(\mathrm{~m}, 2 \mathrm{H}), 7.13-7.08(\mathrm{~m}, 2 \mathrm{H}), 7.01-6.97$ $(\mathrm{m}, 2 \mathrm{H}), 6.83-6.80(\mathrm{~m}, 2 \mathrm{H}), 4.53$ (q, $J=2.1 \mathrm{~Hz}, 1 \mathrm{H}), 3.78(\mathrm{~s}, 3 \mathrm{H}), 2.60$ (ddd, $J=$ 12.7, 8.1, 6.0 Hz, 1H), $2.38(\mathrm{ddd}, J=12.7,8.1,6.9 \mathrm{~Hz}, 1 \mathrm{H}), 2.25$ (d, $J=2.0 \mathrm{~Hz}, 3 \mathrm{H})$, $1.51-1.42(\mathrm{~m}, 2 \mathrm{H}), 0.89(\mathrm{t}, J=7.3 \mathrm{~Hz}, 3 \mathrm{H})$.

${ }^{13} \mathrm{C}$ NMR $\left(101 \mathrm{MHz}, \mathrm{CDCl}_{3}\right) \delta 158.7,148.7,144.8,141.2,139.5,131.7,129.4,126.9$, $125.4,123.5,118.7,114.2,57.8,55.4,35.7,23.5,13.4,11.9$.

HR-ESI-MS m/z calcd for $\mathrm{C}_{20} \mathrm{H}_{23} \mathrm{OS}[\mathrm{M}+\mathrm{H}]^{+}: 311.1464$, found: 311.1463 .

(1-(2-Methoxyphenyl)-3-methyl-1H-inden-2-yl)(propyl)sulfane (3p) 
<smiles>CCCSC1=C(C)c2ccccc2C1c1ccccc1OC</smiles>

Prepared by the method D. Purified by preparative thin layer chromatography (eluent: $\left.\mathrm{PE} / \mathrm{CH}_{2} \mathrm{Cl}_{2}=4 / 1, \mathrm{v} / \mathrm{v}\right)$ to afford $3 \mathbf{p}(19.1 \mathrm{mg}, 62 \%$ yield $)$ as a colorless oil. ${ }^{1} \mathrm{H}$ NMR (400 MHz, $\left.\mathrm{CDCl}_{3}\right) \delta 7.26-7.18(\mathrm{~m}, 4 \mathrm{H}), 7.08-7.05(\mathrm{~m}, 1 \mathrm{H}), 6.99(\mathrm{~d}, J=$ $8.4 \mathrm{~Hz}, 1 \mathrm{H}), 6.82-6.78(\mathrm{~m}, 1 \mathrm{H}), 6.61(\mathrm{~s}, 1 \mathrm{H}), 5.32(\mathrm{~s}, 1 \mathrm{H}), 3.99(\mathrm{~s}, 3 \mathrm{H}), 2.68(\mathrm{dt}, J=$ 13.5, $6.8 \mathrm{~Hz}, 1 \mathrm{H}), 2.41$ (dt, $J=12.9,7.7 \mathrm{~Hz}, 1 \mathrm{H}), 2.27$ (s, 3H), $1.56-1.46(\mathrm{~m}, 2 \mathrm{H})$, $0.91(\mathrm{t}, J=7.3 \mathrm{~Hz}, 3 \mathrm{H})$.

${ }^{13} \mathrm{C} \mathrm{NMR}\left(101 \mathrm{MHz}, \mathrm{CDCl}_{3}\right) \delta 157.8,149.0,148.9,145.1,140.2,139.7,128.1,127.9$, $126.6,124.9,123.2,121.1,118.4,111.0,55.8,35.2,23.7,13.4,11.8$.

HR-ESI-MS m/z calcd for $\mathrm{C}_{20} \mathrm{H}_{23} \mathrm{OS}[\mathrm{M}+\mathrm{H}]^{+}: 311.1464$, found: 311.1461 .

\section{(3,6-Dimethyl-1-phenyl-1H-inden-2-yl)(propyl)sulfane (3q)}<smiles>CCCSC1=C(C)c2ccc(C)cc2C1c1ccccc1</smiles>

Prepared by the method D. Purified by preparative thin layer chromatography (eluent: PE) to afford $\mathbf{3 q}(21.6 \mathrm{mg}, 73 \%$ yield) as a colorless oil. ${ }^{1} \mathrm{H}$ NMR (400 MHz, $\left.\mathrm{CDCl}_{3}\right) \delta 7.31-7.23(\mathrm{~m}, 4 \mathrm{H}), 7.17(\mathrm{~d}, J=7.7 \mathrm{~Hz}, 1 \mathrm{H}), 7.10-$ $7.07(\mathrm{~m}, 3 \mathrm{H}), 6.96(\mathrm{~s}, 1 \mathrm{H}), 4.54(\mathrm{~s}, 1 \mathrm{H}), 2.56(\mathrm{ddd}, J=13.6,8.1,6.0 \mathrm{~Hz}, 1 \mathrm{H}), 2.36-$ $2.33(\mathrm{~m}, 1 \mathrm{H}), 2.29(\mathrm{~s}, 3 \mathrm{H}), 2.25(\mathrm{~d}, J=2.0 \mathrm{~Hz}, 3 \mathrm{H}), 1.50-1.42(\mathrm{~m}, 2 \mathrm{H}), 0.88(\mathrm{t}, J=$ $7.3 \mathrm{~Hz}, 3 \mathrm{H})$.

${ }^{13} \mathrm{C} \mathrm{NMR}\left(101 \mathrm{MHz}, \mathrm{CDCl}_{3}\right) \delta 148.7,142.4,141.9,140.1,137.9,135.3,128.7,128.5$, $127.7,127.0,124.5,118.5,58.5,35.8,23.5,21.6,13.4,11.9$.

HR-ESI-MS m/z calcd for $\mathrm{C}_{20} \mathrm{H}_{23} \mathrm{~S}[\mathrm{M}+\mathrm{H}]^{+}: 295.1515$, found: 295.1516 . 
<smiles>CCCSC1=C(C)c2ccc(C#N)cc2C1c1ccccc1</smiles>

Prepared by the method D. Purified by preparative thin layer chromatography (eluent: $\mathrm{PE} / \mathrm{EtOAc}=10 / 1, \mathrm{v} / \mathrm{v})$ to afford $3 \mathbf{r}(20.6 \mathrm{mg}, 68 \%$ yield $)$ as a colorless oil.

${ }^{1} \mathrm{H}$ NMR (400 MHz, $\left.\mathrm{CDCl}_{3}\right) \delta 7.55(\mathrm{dd}, J=7.9,1.5 \mathrm{~Hz}, 1 \mathrm{H}), 7.34-7.27(\mathrm{~m}, 5 \mathrm{H})$, $7.09-7.06(\mathrm{~m}, 2 \mathrm{H}), 4.65(\mathrm{~s}, 1 \mathrm{H}), 2.67$ (ddd, $J=12.5,8.0,6.0 \mathrm{~Hz}, 1 \mathrm{H}), 2.47-2.40$ (m, 1H), $2.24(\mathrm{~d}, J=1.9 \mathrm{~Hz}, 3 \mathrm{H}), 1.54-1.44(\mathrm{~m}, 2 \mathrm{H}), 0.88(\mathrm{t}, J=7.3 \mathrm{~Hz}, 3 \mathrm{H})$. ${ }^{13} \mathrm{C}$ NMR (101 MHz, $\left.\mathrm{CDCl}_{3}\right) \delta$ 149.2, 148.5, 145.6, 138.2, 137.9, 131.9, 129.2, 128.0, $127.7,126.4,119.9,118.7,107.7,58.2,35.0,23.5,13.3,11.6$.

HR-ESI-MS m/z calcd for $\mathrm{C}_{20} \mathrm{H}_{20} \mathrm{NS}[\mathrm{M}+\mathrm{H}]^{+}: 306.1311$, found: 306.1325 .

(6-Chloro-3-methyl-1-phenyl-1 H-inden-2-yl)(propyl)sulfane (3s)<smiles>CCCSC1=C(C)c2ccc(Cl)cc2C1c1ccccc1</smiles>

Prepared by the method D. Purified by preparative thin layer chromatography (eluent: PE) to afford $3 \mathbf{s}(25.3 \mathrm{mg}, 81 \%$ yield) as a colorless oil.

${ }^{1} \mathrm{H}$ NMR (400 MHz, $\left.\mathrm{CDCl}_{3}\right) \delta 7.32-7.27(\mathrm{~m}, 3 \mathrm{H}), 7.26-7.23(\mathrm{~m}, 2 \mathrm{H}), 7.16(\mathrm{~d}, J=$ $8.0 \mathrm{~Hz}, 1 \mathrm{H}), 7.09-7.04(\mathrm{~m}, 3 \mathrm{H}), 4.56(\mathrm{~d}, J=2.2 \mathrm{~Hz}, 1 \mathrm{H}), 2.61-2.54(\mathrm{~m}, 1 \mathrm{H}), 2.36$ (ddd, $J=12.6,8.1,6.9 \mathrm{~Hz}, 1 \mathrm{H}), 2.23$ (d, $J=2.1 \mathrm{~Hz}, 3 \mathrm{H}), 1.50-1.42$ (m, 2H), 0.87 (t, $J=7.3 \mathrm{~Hz}, 3 \mathrm{H})$.

${ }^{13} \mathrm{C}$ NMR $\left(101 \mathrm{MHz}, \mathrm{CDCl}_{3}\right) \delta 149.9,143.4,140.4,140.0,138.9,131.4,129.0,128.4$, $127.4,127.2,124.1,119.5,58.5,35.6,23.5,13.3,11.8$.

HR-ESI-MS m/z calcd for $\mathrm{C}_{19} \mathrm{H}_{20} \mathrm{SCl}[\mathrm{M}+\mathrm{H}]^{+}: 315.0969$, found: 315.0975 . 
<smiles>CCCSC1=C(C)c2ccc(F)cc2C1c1ccccc1</smiles>

Prepared by the method D. Purified by preparative thin layer chromatography (eluent:

PE) to afford $3 \mathrm{t}$ (24.3 $\mathrm{mg}, 82 \%$ yield) as a colorless oil.

${ }^{1} \mathrm{H}$ NMR (400 MHz, $\left.\mathrm{CDCl}_{3}\right) \delta 7.32-7.25(\mathrm{~m}, 3 \mathrm{H}), 7.19(\mathrm{dd}, J=8.3,5.0 \mathrm{~Hz}, 1 \mathrm{H})$, $7.08-7.06(\mathrm{~m}, 2 \mathrm{H}), 7.00-6.95(\mathrm{~m}, 1 \mathrm{H}), 6.86(\mathrm{dd}, J=8.6,2.3 \mathrm{~Hz}, 1 \mathrm{H}), 4.55(\mathrm{~s}, 1 \mathrm{H})$, $2.56(\mathrm{ddd}, J=12.8,8.1,6.1 \mathrm{~Hz}, 1 \mathrm{H}), 2.39-2.32(\mathrm{~m}, 1 \mathrm{H}), 2.26(\mathrm{~d}, J=2.0 \mathrm{~Hz}, 3 \mathrm{H})$, $1.52-1.43(\mathrm{~m}, 2 \mathrm{H}), 0.88(\mathrm{t}, J=7.3 \mathrm{~Hz}, 3 \mathrm{H})$.

${ }^{13} \mathrm{C} \mathrm{NMR}\left(101 \mathrm{MHz}, \mathrm{CDCl}_{3}\right) \delta 161.9(\mathrm{~d}, J=244.2 \mathrm{~Hz}), 150.4(\mathrm{~d}, J=8.1 \mathrm{~Hz}), 141.1$, $140.9(\mathrm{~d}, J=2.5 \mathrm{~Hz}), 139.2,138.7$ (d, $J=3.9 \mathrm{~Hz}), 128.9,128.4,127.3,119.4$ (d, $J=$ 8.6 Hz), 113.8 (d, $J=22.7 \mathrm{~Hz}), 111.5(\mathrm{~d}, J=23.5 \mathrm{~Hz}), 58.6$ (d, $J=2.3 \mathrm{~Hz}), 35.8,23.5$, $13.3,12.0$.

HR-ESI-MS m/z calcd for $\mathrm{C}_{19} \mathrm{H}_{20} \mathrm{SF}[\mathrm{M}+\mathrm{H}]^{+}: 299.1264$, found: 299.1269 .

\section{(5-Fluoro-3-methyl-1-phenyl-1H-inden-2-yl)(propyl)sulfane (3u)}<smiles>CCCSC1=C(C)c2cc(F)ccc2C1c1ccccc1</smiles>

Prepared by the method D. Purified by preparative thin layer chromatography (eluent: $\mathrm{PE})$ to afford $3 \mathbf{u}(25.5 \mathrm{mg}, 86 \%$ yield $)$ as a colorless oil. ${ }^{1} \mathrm{H} \mathrm{NMR}\left(400 \mathrm{MHz}, \mathrm{CDCl}_{3}\right) \delta 7.31-7.24(\mathrm{~m}, 3 \mathrm{H}), 7.09-7.06(\mathrm{~m}, 2 \mathrm{H}), 7.03(\mathrm{dd}, J=$ 8.2, $5.1 \mathrm{~Hz}, 1 \mathrm{H}), 6.95(\mathrm{dd}, J=9.0,2.3 \mathrm{~Hz}, 1 \mathrm{H}), 6.80-6.75(\mathrm{~m}, 1 \mathrm{H}), 4.56(\mathrm{~s}, 1 \mathrm{H})$, 2.61 (ddd, $J=12.6,8.0,6.0 \mathrm{~Hz}, 1 \mathrm{H}), 2.42-2.36(\mathrm{~m}, 1 \mathrm{H}), 2.23(\mathrm{~d}, J=1.9 \mathrm{~Hz}, 3 \mathrm{H})$, $1.53-1.44(\mathrm{~m}, 2 \mathrm{H}), 0.89(\mathrm{t}, J=7.3 \mathrm{~Hz}, 3 \mathrm{H})$. 
${ }^{13} \mathrm{C}$ NMR (101 MHz, $\left.\mathrm{CDCl}_{3}\right) \delta 162.9(\mathrm{~d}, J=243.3 \mathrm{~Hz}), 146.9$ (d, $\left.J=8.6 \mathrm{~Hz}\right), 143.7$ $(\mathrm{d}, J=2.7 \mathrm{~Hz}), 142.0,140.1(\mathrm{~d}, J=3.0 \mathrm{~Hz}), 139.5,128.9,128.3,127.2,124.3$ (d, $J=$ $9.0 \mathrm{~Hz}), 111.6(\mathrm{~d}, J=23.1 \mathrm{~Hz}), 105.9(\mathrm{~d}, J=23.4 \mathrm{~Hz}), 58.0,35.5,23.5,13.3,11.8$.

HR-ESI-MS m/z calcd for $\mathrm{C}_{19} \mathrm{H}_{20} \mathrm{SF}[\mathrm{M}+\mathrm{H}]^{+}: 299.1264$, found: 299.1261 .

\section{(1,3-Diphenyl-1H-inden-2-yl)(propyl)sulfane (3v)}

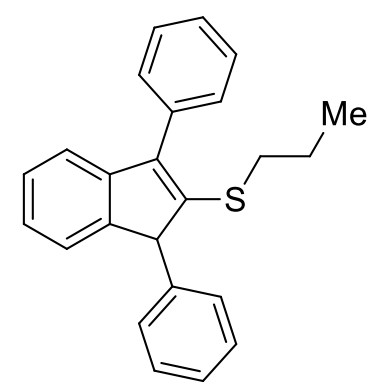

Prepared by the method D. Purified by preparative thin layer chromatography (eluent: PE) to afford $\mathbf{3 v}(20.7 \mathrm{mg}, 61 \%$ yield $)$ as a colorless oil.

${ }^{1} \mathrm{H}$ NMR (400 MHz, $\left.\mathrm{CDCl}_{3}\right) \delta 7.60(\mathrm{~d}, J=7.6 \mathrm{~Hz}, 2 \mathrm{H}), 7.54-7.50$ (m, 2H), $7.43-$ $7.40(\mathrm{~m}, 1 \mathrm{H}), 7.37-7.33(\mathrm{~m}, 2 \mathrm{H}), 7.30-7.18(\mathrm{~m}, 7 \mathrm{H}), 7.13-7.09(\mathrm{~m}, 1 \mathrm{H}), 4.80(\mathrm{~s}$, 1H), $2.64(\mathrm{dt}, J=13.3,7.0 \mathrm{~Hz}, 1 \mathrm{H}), 2.38(\mathrm{dt}, J=13.4,7.6 \mathrm{~Hz}, 1 \mathrm{H}), 1.49-1.39(\mathrm{~m}$, $2 \mathrm{H}), 0.80(\mathrm{t}, J=7.4 \mathrm{~Hz}, 3 \mathrm{H})$.

${ }^{13} \mathrm{C}$ NMR (101 MHz, $\left.\mathrm{CDCl}_{3}\right) \delta 148.2,144.1,142.5,141.9,139.7,134.7,129.4,129.0$, $128.5,128.3,127.9,127.2,127.0,125.1,123.8,119.5,58.8,34.9,23.2,13.4$.

HR-ESI-MS m/z calcd for $\mathrm{C}_{24} \mathrm{H}_{23} \mathrm{~S}[\mathrm{M}+\mathrm{H}]^{+}: 343.1515$, found: 343.1521 .

(3-Phenyl-1-propyl-1 H-inden-2-yl)(propyl)sulfane (3w)<smiles>CCCSC1=C(c2ccccc2)c2ccccc2C1CCC</smiles> 
Prepared by the method D. Purified by preparative thin layer chromatography (eluent: PE) to afford $3 \mathbf{w}(20.5 \mathrm{mg}, 67 \%$ yield) as a colorless oil.

${ }^{1} \mathrm{H}$ NMR (400 MHz, $\left.\mathrm{CDCl}_{3}\right) \delta 7.48-7.44(\mathrm{~m}, 5 \mathrm{H}), 7.41-7.36(\mathrm{~m}, 1 \mathrm{H}), 7.25-7.18$ (m, 3H), $3.73(\mathrm{dd}, J=6.6,4.1 \mathrm{~Hz}, 1 \mathrm{H}), 2.76(\mathrm{ddd}, J=12.6,8.1,6.0 \mathrm{~Hz}, 1 \mathrm{H}), 2.58$ (ddd, $J=12.6,8.2,7.1 \mathrm{~Hz}, 1 \mathrm{H}), 2.13(\mathrm{ddt}, J=13.5,11.5,4.7 \mathrm{~Hz}, 1 \mathrm{H}), 1.87$ (dddd, $J=$ 13.6, 11.2, 6.6, $4.6 \mathrm{~Hz}, 1 \mathrm{H}), 1.58-1.51(\mathrm{~m}, 2 \mathrm{H}), 1.38-1.32(\mathrm{~m}, 1 \mathrm{H}), 1.09-1.02(\mathrm{~m}$, $1 \mathrm{H}), 0.93-0.88(\mathrm{~m}, 6 \mathrm{H})$.

${ }^{13} \mathrm{C}$ NMR $\left(101 \mathrm{MHz}, \mathrm{CDCl}_{3}\right) \delta 147.2,144.9,142.8,141.7,135.0,129.5,128.4,127.6$, $126.7,124.6,123.1,119.6,51.1,35.7,34.1,23.5,18.1,14.5,13.4$.

HR-ESI-MS m/z calcd for $\mathrm{C}_{21} \mathrm{H}_{25} \mathrm{~S}[\mathrm{M}+\mathrm{H}]^{+}: 309.1671$, found: 309.1677 .

\section{(3-Ethyl-1-pentyl-1 $H$-inden-2-yl)(p-tolyl)s ulfane (3x)}<smiles>CCCCCC1C(Sc2ccc(C)cc2)=C(CC)c2ccccc21</smiles>

Prepared by the method D. Purified by preparative thin layer chromatography (eluent: PE) to afford 3x (33.3 mg, 99\% yield) as a colorless oil. ${ }^{1} \mathrm{H}$ NMR (400 MHz, $\left.\mathrm{CDCl}_{3}\right) \delta 7.38-7.35(\mathrm{~m}, 2 \mathrm{H}), 7.33-7.27(\mathrm{~m}, 2 \mathrm{H}), 7.21(\mathrm{~d}, J=$ $8.0 \mathrm{~Hz}, 2 \mathrm{H}), 7.06$ (d, $J=7.9 \mathrm{~Hz}, 2 \mathrm{H}), 3.48(\mathrm{t}, J=5.5 \mathrm{~Hz}, 1 \mathrm{H}), 2.80$ (q, $J=7.6 \mathrm{~Hz}$, 2H), $2.31(\mathrm{~s}, 3 \mathrm{H}), 1.93(\mathrm{ddt}, J=14.8,9.4,4.7 \mathrm{~Hz}, 1 \mathrm{H}), 1.70(\mathrm{dtd}, J=11.6,6.7,2.4 \mathrm{~Hz}$, $1 \mathrm{H}), 1.22(\mathrm{t}, J=7.6 \mathrm{~Hz}, 3 \mathrm{H}), 1.18-1.12(\mathrm{~m}, 4 \mathrm{H}), 0.93-0.85(\mathrm{~m}, 2 \mathrm{H}), 0.81(\mathrm{t}, J=6.9$ $\mathrm{Hz}, 3 \mathrm{H})$.

${ }^{13} \mathrm{C}$ NMR $\left(101 \mathrm{MHz}, \mathrm{CDCl}_{3}\right) \delta 149.4,148.1,144.1,136.9,136.2,133.3,129.9,129.8$, $126.5,125.2,123.2,119.3,50.4,32.2,30.7,24.1,22.5,21.1,19.9,14.2,13.8$.

HR-APCI-MS m/z calcd for $\mathrm{C}_{23} \mathrm{H}_{27} \mathrm{~S}$ [M-H]-: 335.1839, found: 335.1838 . 


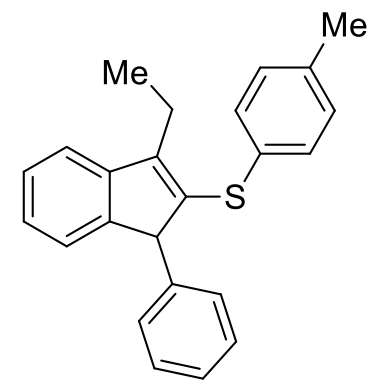

Prepared by the method D. Purified by preparative thin layer chromatography (eluent: PE) to afford $\mathbf{3 y}(20.5 \mathrm{mg}, 60 \%$ yield) as a colorless oil.

${ }^{1} \mathrm{H}$ NMR (400 MHz, $\left.\mathrm{CDCl}_{3}\right) \delta 7.41-7.38(\mathrm{~m}, 1 \mathrm{H}), 7.32-7.28(\mathrm{~m}, 1 \mathrm{H}), 7.20-7.17$ (m, 3H), $7.15-7.11(\mathrm{~m}, 2 \mathrm{H}), 7.08-7.05(\mathrm{~m}, 2 \mathrm{H}), 7.03-6.99(\mathrm{~m}, 2 \mathrm{H}), 6.82-6.80$ (m, 2H), $4.49(\mathrm{~s}, 1 \mathrm{H}), 2.83(\mathrm{qd}, J=7.6,1.1 \mathrm{~Hz}, 2 \mathrm{H}), 2.31(\mathrm{~s}, 3 \mathrm{H}), 1.28(\mathrm{t}, J=7.6 \mathrm{~Hz}$, $3 \mathrm{H})$.

${ }^{13} \mathrm{C}$ NMR (101 MHz, $\left.\mathrm{CDCl}_{3}\right) \delta 149.3,149.0,143.8,139.5,138.3,136.6,132.5,130.8$, 129.7, 128.6, 128.5, 127.0, 126.9, 125.9, 124.2, 119.4, 57.7, 21.2, 20.0, 13.7.

HR-APCI-MS m/z calcd for $\mathrm{C}_{24} \mathrm{H}_{21} \mathrm{~S}$ [M-H]-: 341.1369, found: 341.1367.

(5-Methoxy-1-methyl-1-phenyl-1 H-inden-2-yl)(propyl)sulfane (3z)<smiles>CCCSC1=Cc2cc(OC)ccc2C1(C)c1ccccc1</smiles>

Prepared by the method D. Purified by preparative thin layer chromatography (eluent: $\left.\mathrm{PE} / \mathrm{CH}_{2} \mathrm{Cl}_{2}=4: 1, \mathrm{v} / \mathrm{v}\right)$ to afford $\mathbf{3 z}(19.0 \mathrm{mg}, 61 \%$ yield) as a colorless oil. ${ }^{1} \mathrm{H} \mathrm{NMR}\left(400 \mathrm{MHz}, \mathrm{CDCl}_{3}\right) \delta 7.24(\mathrm{~d}, J=7.5 \mathrm{~Hz}, 2 \mathrm{H}), 7.20(\mathrm{~d}, J=6.9 \mathrm{~Hz}, 1 \mathrm{H}), 7.15$ $(\mathrm{d}, J=6.8 \mathrm{~Hz}, 2 \mathrm{H}), 6.93(\mathrm{~d}, J=8.1 \mathrm{~Hz}, 1 \mathrm{H}), 6.82(\mathrm{~d}, J=2.3 \mathrm{~Hz}, 1 \mathrm{H}), 6.58(\mathrm{dd}, J=$ 8.2, $2.4 \mathrm{~Hz}, 1 \mathrm{H}), 6.36(\mathrm{~s}, 1 \mathrm{H}), 3.82(\mathrm{~s}, 3 \mathrm{H}), 2.85(\mathrm{t}, J=7.5 \mathrm{~Hz}, 2 \mathrm{H}), 1.75-1.70(\mathrm{~m}$, $5 \mathrm{H}), 1.02(\mathrm{t}, J=7.4 \mathrm{~Hz}, 3 \mathrm{H})$. 
${ }^{13} \mathrm{C}$ NMR $\left(101 \mathrm{MHz}, \mathrm{CDCl}_{3}\right) \delta 159.3,158.1,146.7,144.2,142.7,128.4,126.7,126.5$, $122.9,120.3,109.3,105.4,58.6,55.5,34.2,23.7,22.0,13.8$.

HR-ESI-MS m/z calcd for $\mathrm{C}_{20} \mathrm{H}_{23} \mathrm{OS}[\mathrm{M}+\mathrm{H}]^{+}: 311.1464$, found: 311.1473 .

\section{(1-(4-Methoxyphenyl)-3-methyl-1H-inden-2-yl)(p-tolyl)sulfane (3aa)}<smiles></smiles>

Prepared by the method D. Purified by preparative thin layer chromatography (eluent: $\left.\mathrm{PE} / \mathrm{CH}_{2} \mathrm{Cl}_{2}=4: 1, \mathrm{v} / \mathrm{v}\right)$ to afford 3aa $(21.5 \mathrm{mg}, 60 \%$ yield) as a light yellow oil. ${ }^{1} \mathrm{H}$ NMR (400 MHz, $\left.\mathrm{CDCl}_{3}\right) \delta 7.39-7.31(\mathrm{~m}, 2 \mathrm{H}), 7.20-7.13(\mathrm{~m}, 2 \mathrm{H}), 7.12-7.09$ (m, 2H), $7.04(\mathrm{~d}, J=8.1 \mathrm{~Hz}, 2 \mathrm{H}), 6.80-6.75(\mathrm{~m}, 4 \mathrm{H}), 4.51(\mathrm{q}, J=2.1 \mathrm{~Hz}, 1 \mathrm{H}), 3.78$ (s, 3H), $2.33-2.33(\mathrm{~m}, 6 \mathrm{H})$.

${ }^{13} \mathrm{C} \mathrm{NMR}\left(101 \mathrm{MHz}, \mathrm{CDCl}_{3}\right) \delta 158.6,148.7,144.7,143.3,139.0,136.3,132.6,131.2$, $130.4,129.7,129.6,127.0,126.0,123.9,119.2,113.9,57.3,55.3,21.2,12.0$.

HR-ESI-MS m/z calcd for $\mathrm{C}_{24} \mathrm{H}_{21} \mathrm{OS}$ [M-H]-: 357.1319, found: 357.1319.

HPLC (OD-H, $i$-PrOH $/ n$-hexane $=0.8 / 99.2$, flow rate $=0.4 \mathrm{~mL} / \mathrm{min}, \mathrm{I}=230 \mathrm{~nm}) \mathrm{tR}$ $=15.9 \mathrm{~min}, 20.6 \mathrm{~min}$.

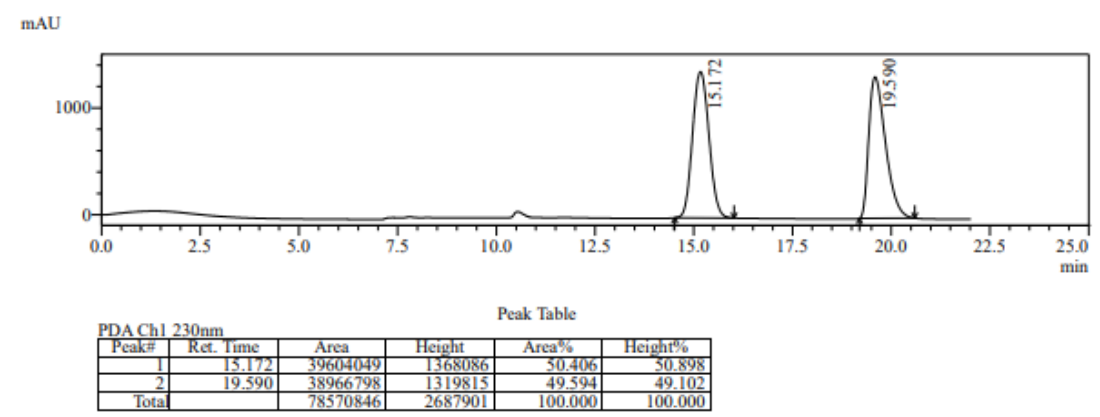



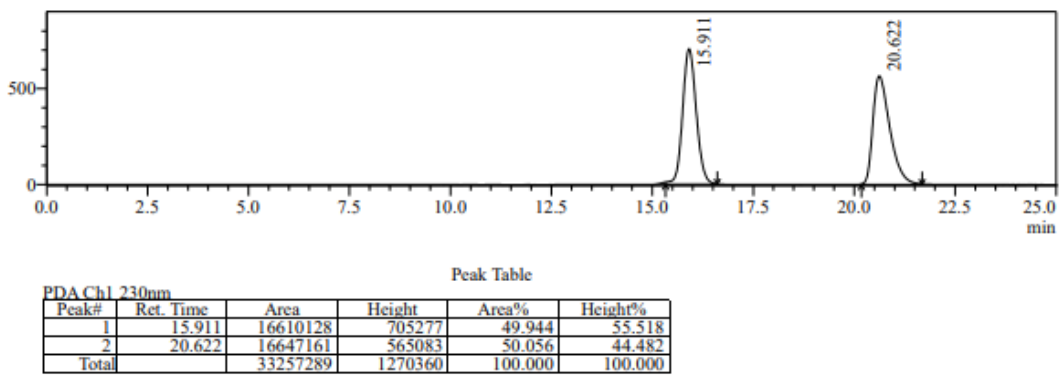

(E)-(4-Azido-2-phenyloct-2-en-3-yl)(propyl)sulfane (4)<smiles>CCCCC(N)/C(SCCC)=C(\C)c1ccccc1</smiles>

Prepared by the method E. Purified by preparative thin layer chromatography (eluent: PE) to afford 4 (15.8 mg, 52\% yield) as a colorless oil.

${ }^{1} \mathrm{H}$ NMR (400 MHz, $\left.\mathrm{CDCl}_{3}\right) \delta 7.38-7.34(\mathrm{~m}, 2 \mathrm{H}), 7.29$ (d, J=6.9 Hz, 1H), 7.08 (d, $J=6.5 \mathrm{~Hz}, 2 \mathrm{H}), 4.14(\mathrm{t}, J=7.1 \mathrm{~Hz}, 1 \mathrm{H}), 2.79(\mathrm{qd}, J=7.7,2.8 \mathrm{~Hz}, 2 \mathrm{H}), 2.36(\mathrm{~s}, 3 \mathrm{H})$, $1.73-1.65(\mathrm{~m}, 4 \mathrm{H}), 1.23-1.11(\mathrm{~m}, 4 \mathrm{H}), 1.06(\mathrm{t}, J=7.1 \mathrm{~Hz}, 3 \mathrm{H}), 0.84(\mathrm{t}, J=6.7 \mathrm{~Hz}$, $3 \mathrm{H})$.

${ }^{13} \mathrm{C}$ NMR $\left(101 \mathrm{MHz}, \mathrm{CDCl}_{3}\right) \delta 149.5,142.6,131.5,128.7,127.7,127.3,64.8,37.9$, $32.7,28.3,25.0,23.4,22.5,14.0,13.8$.

HR-ESI-MS m/z calcd for $\mathrm{C}_{17} \mathrm{H}_{26} \mathrm{~N}_{3} \mathrm{~S}[\mathrm{M}+\mathrm{H}]^{+}: 304.1842$, found: 304.1847.

\section{(1-Butyl-3-methyl-1 H-inden-2-yl)(propyl)sulfane (4')}<smiles>CCCCC1C(SCCC)=C(C)c2ccccc21</smiles>

Prepared by the method E. Purified by preparative thin layer chromatography (eluent: PE) to afford 4' (5.2 mg, 20\% yield) as a a light yellow oil. 
${ }^{1} \mathrm{H}$ NMR (400 MHz, $\left.\mathrm{CDCl}_{3}\right) \delta 7.40-7.37(\mathrm{~m}, 1 \mathrm{H}), 7.31-7.26(\mathrm{~m}, 2 \mathrm{H}), 7.18(\mathrm{ddd}, J$ $=7.3,6.8,1.8 \mathrm{~Hz}, 1 \mathrm{H}), 3.53-3.50(\mathrm{~m}, 1 \mathrm{H}), 2.81-2.74(\mathrm{~m}, 1 \mathrm{H}), 2.62(\mathrm{ddd}, J=12.7$, 8.0, 7.3 Hz, 1H), 2.19 (d, $J=2.0 \mathrm{~Hz}, 3 \mathrm{H}), 2.10-2.02(\mathrm{~m}, 1 \mathrm{H}), 1.77-1.70(\mathrm{~m}, 1 \mathrm{H})$, $1.61-1.54(\mathrm{~m}, 2 \mathrm{H}), 1.48-1.38(\mathrm{~m}, 1 \mathrm{H}), 1.29-1.22(\mathrm{~m}, 2 \mathrm{H}), 1.17-1.09(\mathrm{~m}, 1 \mathrm{H})$, 0.98 (t, $J=7.3 \mathrm{~Hz}, 3 \mathrm{H}), 0.82(\mathrm{t}, J=7.3 \mathrm{~Hz}, 3 \mathrm{H})$.

${ }^{13} \mathrm{C} \mathrm{NMR}\left(101 \mathrm{MHz}, \mathrm{CDCl}_{3}\right) \delta 147.4,145.5,140.9,139.0,126.6,124.7,122.8,118.7$, $50.6,36.3,30.9,27.0,23.7,23.2,14.2,13.4,11.8$.

HR-APCI-MS m/z calcd for $\mathrm{C}_{17} \mathrm{H}_{23} \mathrm{~S}$ [M-H]-: 259.1526, found: 259.1527.

\section{References}

(1) (a) Taniguchi, N. Convenient Synthesis of Unsymmetrical Organochalcogenides Using Organoboronic Acids with Dichalcogenides via Cleavage of the $\mathrm{S}-\mathrm{S}$, Se-Se, or Te-Te Bond by a Copper Catalyst. J. Org. Chem. 2007, 72, 1241-1245. (b) Prasad, C. D.; Balkrishna, S. J.; Kumar, A.; Bhakuni, B. S.; Shrimali, K.; Biswas, S.; Kumar, S. Transition-Metal-Free Synthes is of Unsymmetrical Diaryl Chalcogenides from Arenes and Diaryl Dichalcogenides. J. Org. Chem. 2013, 78, 1434-1443.

(2) (a) Kumar, A.; Kumar, S. A Convenient and Efficient Copper-Catalyzed Synthesis of Unsymmetrical and Symmetrical Diaryl Chalcogenides from Arylboronic Acids in Ethanol at Room Temperature. Tetrahedron 2014, 70, 1763-1772. (b) Yu, J.-T.; Guo, H.; Yi, Y.; Fei, H.; Jiang, Y. The Chan-Lam Reaction of Chalcogen Elements Leading to Aryl Chalcogenides. Adv. Synth. Catal. 2014, 356, 749-752.

(3) Ting, C.-M.; Hsu, Y.-L.; Liu, R.-S. Gold-Catalyzed Isomerization of Unactivated Allenes into 1,3-Dienes under Ambient Conditions. Chem. Commun. 2012, 48, 6577-6579.

(4) Zheng, Y.; Miao, B.; Qin, A.; Xiao, J.; Liu, Q.; Li, G.; Zhang, L.; Zhang, F.; Guo, Y.; Ma, S. Negishi Coupling for Highly Selective Syntheses of Allenes via Ligand Effect and Mechanistic Study via SAESI-MS/MS. Chin. J. Chem. 2019, 37, 
1003-1008.

(5) He, Q.; Ma, S. A 1,3-Lithium Shift of Propargylic/Allenylic Lithium and the Subsequent Transmetalation Coupling Reaction with Aryl Halides. Angew. Chem. Int. Ed. 2004, 43, 988-990.

(6) Li, Q.-H.; Jeng, J.-Y.; Gau, H.-M. Highly Efficient Synthesis of Allenes from Trimethylaluminum Reagent and Propargyl Acetates Mediated by a Palladium Catalyst. Eur. J. Org. Chem. 2014, 7916-7923.

(7) Shao, X. B.; Zhang, Z.; Li, Q. H.; Zhao, Z. G. Synthesis of multi-substituted allenes from organoalane reagents and propargyl esters by using a nickel catalyst. Org. Biomol. Chem. 2018, 16, 4797-4806.

(8) Liu, Z.; Liao, P.; Bi, X. Lewis and Brønsted Acid Cocatalyzed Reductive Deoxyallenylation of Propargylic Alcohols with 2-Nitrobenzenesulfonylhydrazide. Chem. Eur. J. 2014, 20, 17277-17281.

(9) Xiao, Q.; Xia, Y.; Li, H.; Zhang, Y.; Wang, J. Coupling of $N$-Tosylhydrazones with Terminal Alkynes Catalyzed by Copper(I): Synthesis of Trisubstituted Allenes. Angew. Chem. Int. Ed. 2011, 50, 1114-1117.

(10)Zhang, H.; Fu, X.; Chen, J.; Wang, E.; Liu, Y.; Li, Y. Generation of Allenic/Propargylic Zirconium Complexes and Subsequent Cross-Coupling Reactions: A Facile Synthesis of Multisubstituted Allenes. J. Org. Chem. 2009, 74, 9351-9358.

(11)Reese, C. B.; Yan, H. Solution Phase Synthesis of ISIS 2922 (Vitravene) by the Modified H-phosphonate Approach. J. Chem. Soc., Perkin Trans. 1 2002, 2619-2633.

(12)Liang, Y.; Zhao, X. Enantioselective Construction of Chiral Sulfides via Catalytic Electrophilic Azidothiolation and Oxythiolation of $N$-Allyl Sulfonamides. $A C S$ Catal. 2019, 9, 6896-6902.

(13)Ramesh, E.; Guntreddi, T.; Sahoo, A. K. $\mathrm{AlCl}_{3}$-Catalyzed Intermolecular Annulation of Thiol Derivatives and Alkynes by 1,2-Sulfur Migration: Construction of 6-Substituted Benzo $[b]$ thiophenes. Eur. J. Org. Chem. 2017, 4405-4413. 
(14)Hostier, T.; Ferey, V.; Ricci, G; Pardo, D. G.; Cossy, J. Synthesis of Aryl Sulfides: Metal-Free C-H Sulfenylation of Electron-Rich Arenes. Org. Lett. 2015, 17, 3898-3901.

(15)Ramesh, E.; Shankar, M.; Dana, S.; Sahoo, A. K. Silver-Mediated Oxidative Annulation of $N$-Arylthio Succinimides with Alkynes: Direct Access to Benzo[b]thiophenes. Org. Chem. Front. 2016, 3, 1126-1130.

(16) Xu, C.; Ma, B.; Shen, Q. N-Trifluoromethylthiosaccharin: An Easily Accessible, Shelf-Stable, Broadly Applicable Trifluoro-Methylthiolating Reagent. Angew. Chem. Int. Ed. 2014, 53, 9316-9320.

(17)Luo, J.; Liu, Y.; Zhao, X. Chiral Selenide-Catalyzed Enantioselective Construction of Saturated Trifluoromethylthiolated Azaheterocycles. Org. Lett. 2017, 19, 3434-3437. 


\section{NMR spectra for new compounds}

${ }^{1} \mathrm{H} \mathrm{NMR}\left(400 \mathrm{MHz}, \mathrm{CDCl}_{3}\right)$ spectrum of compound $\mathbf{1 e}$

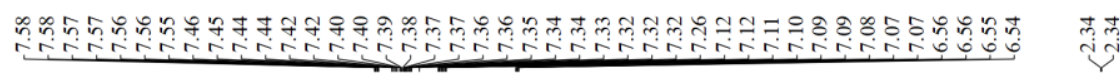
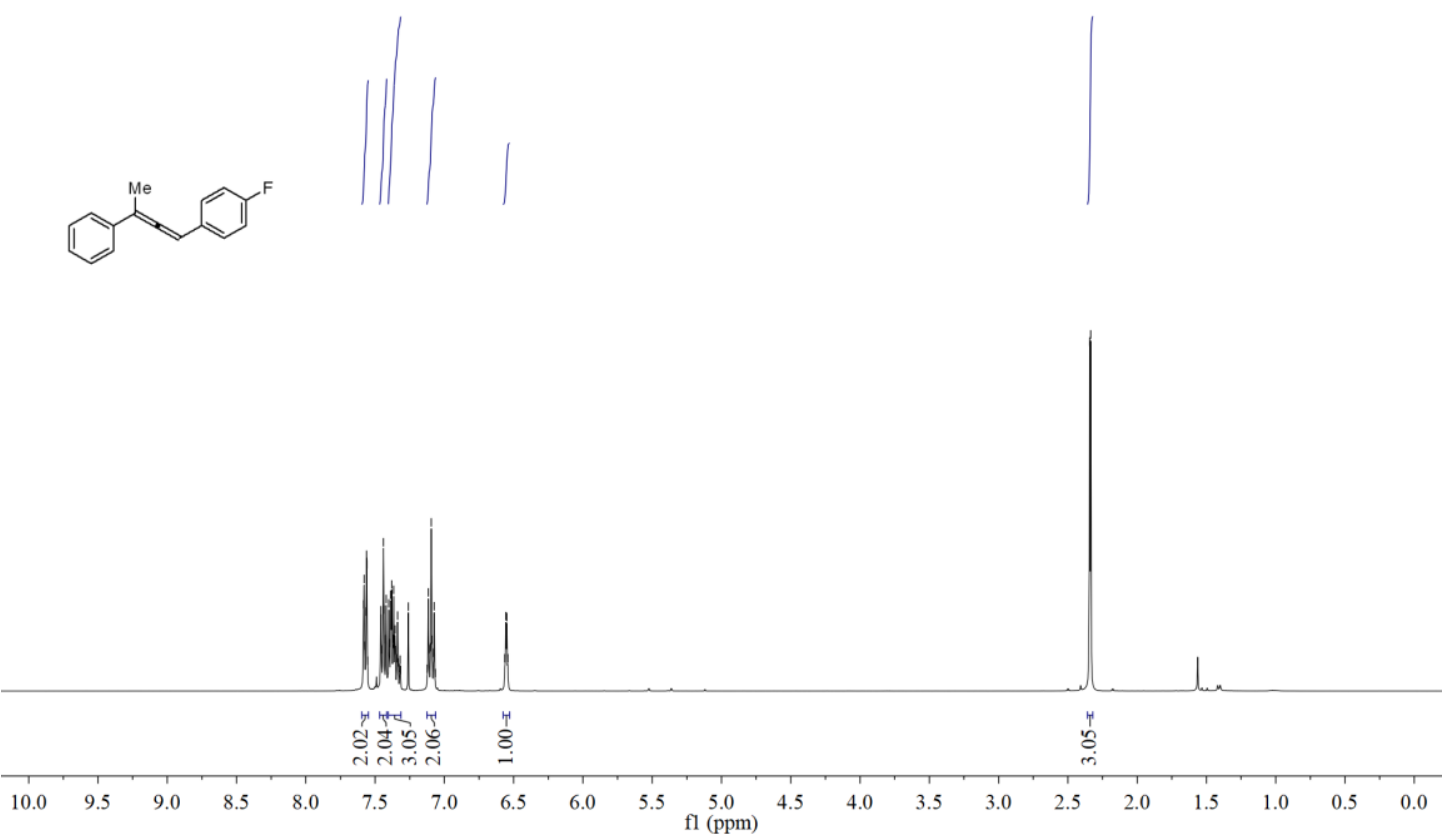

${ }^{13} \mathrm{C} \mathrm{NMR}\left(101 \mathrm{MHz}, \mathrm{CDCl}_{3}\right)$ spectrum of compound $\mathbf{1 e}$

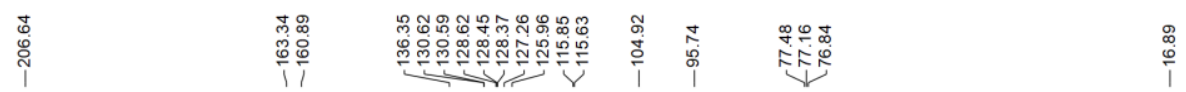

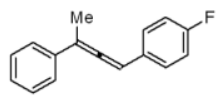

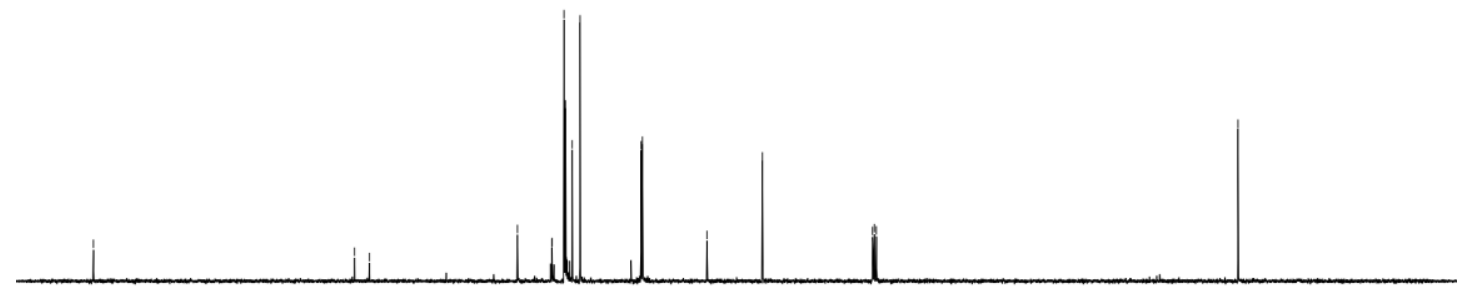


${ }^{1} \mathrm{H}$ NMR (400 MHz, $\left.\mathrm{CDCl}_{3}\right)$ spectrum of compound $\mathbf{1 i}$

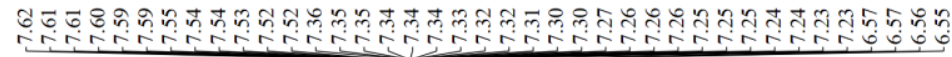
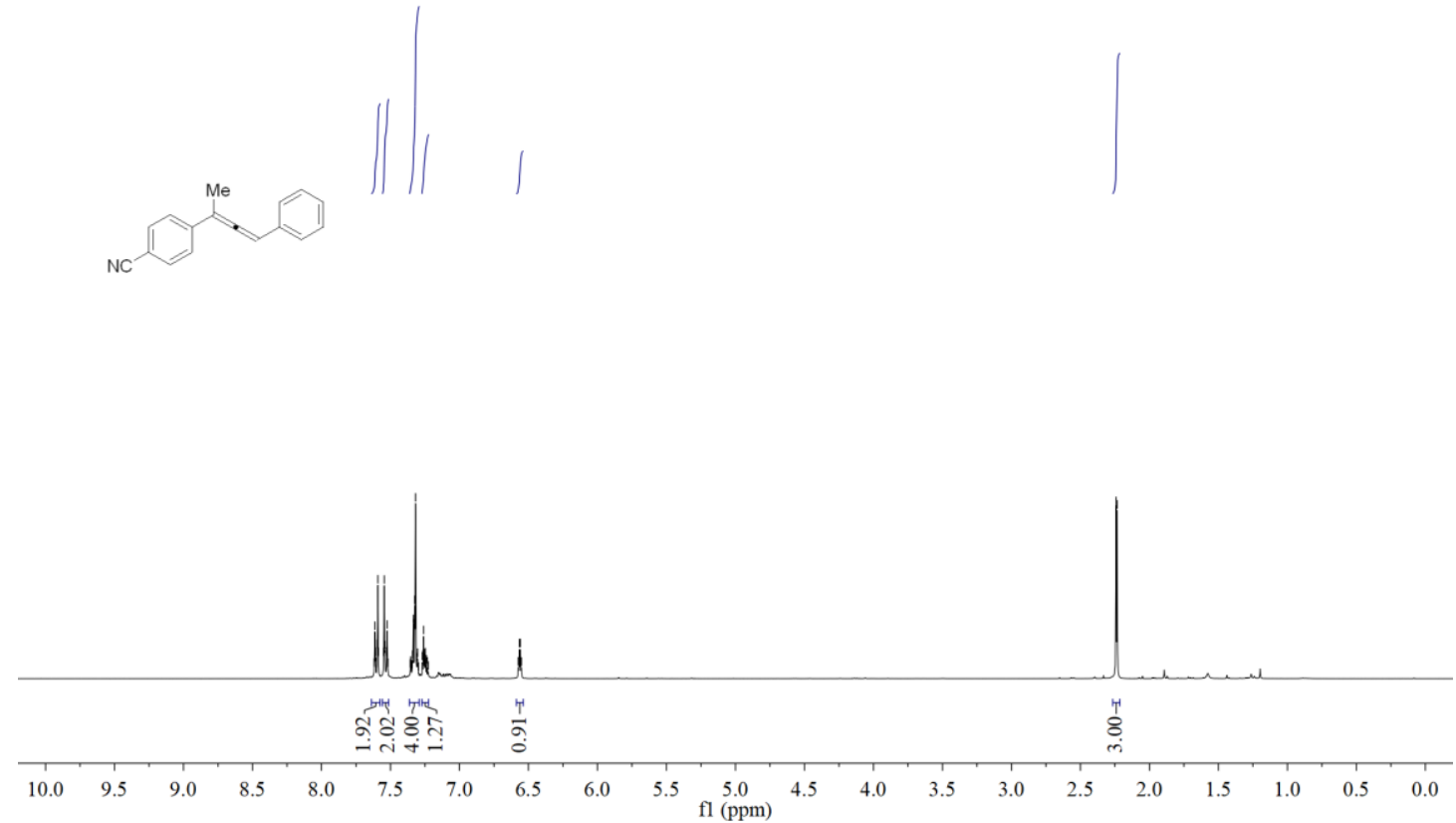

${ }^{13} \mathrm{C} \mathrm{NMR}\left(101 \mathrm{MHz}, \mathrm{CDCl}_{3}\right)$ spectrum of compound $\mathbf{1 i}$
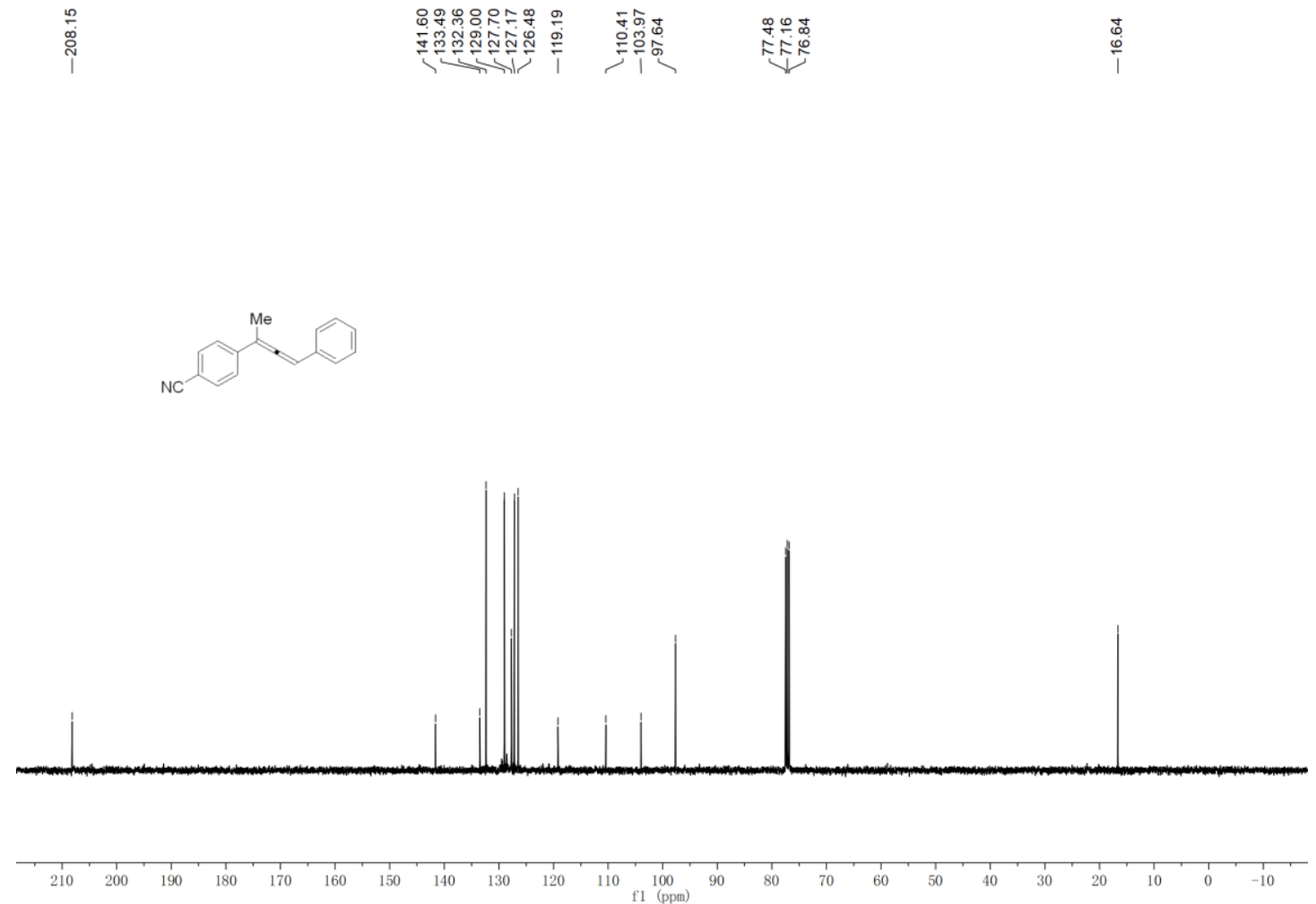
${ }^{1} \mathrm{H}$ NMR (400 MHz, $\left.\mathrm{CDCl}_{3}\right)$ spectrum of compound $\mathbf{1 l}$

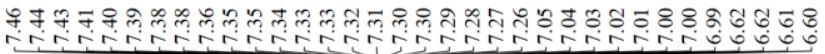
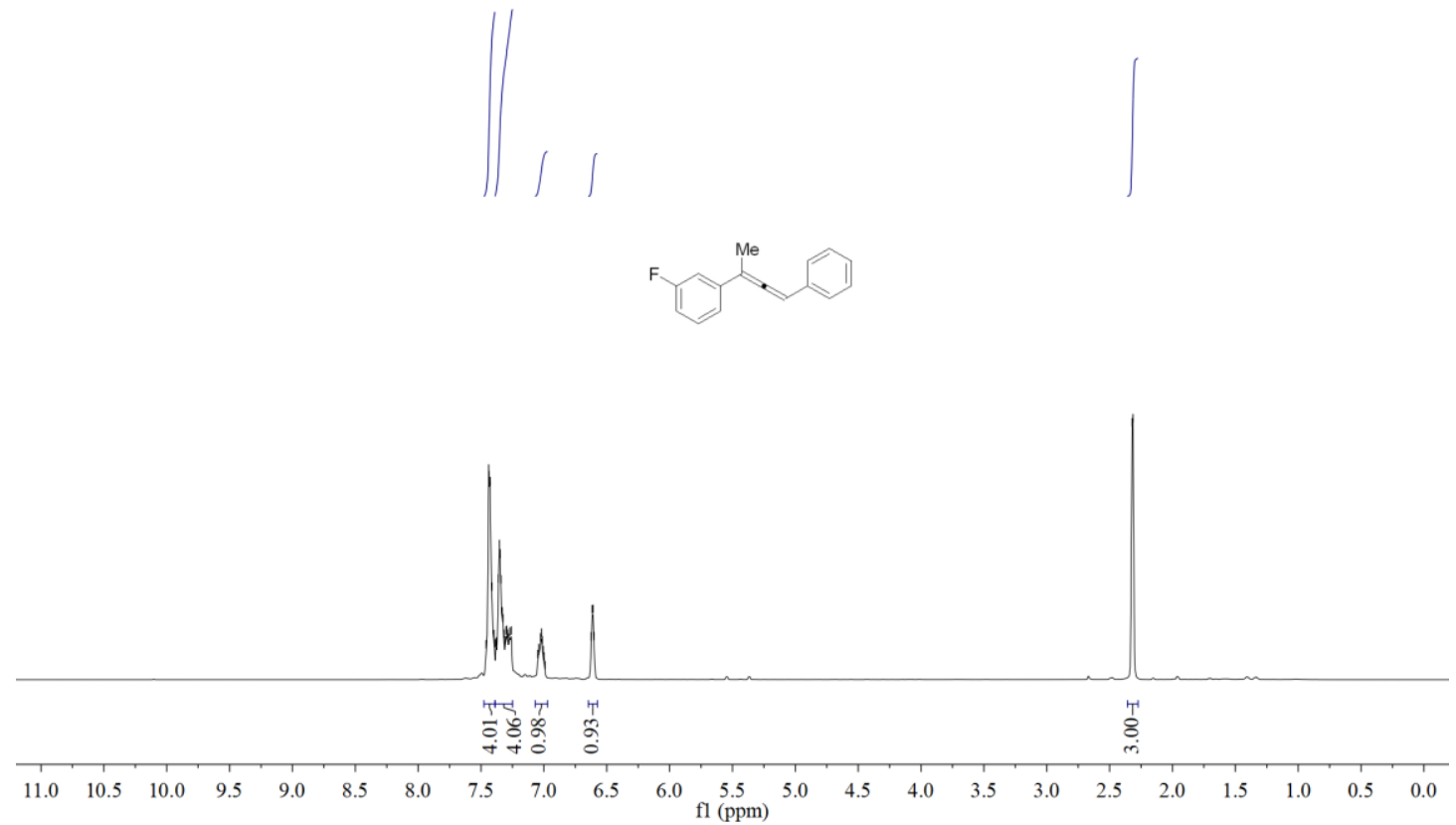

${ }^{13} \mathrm{C} \mathrm{NMR}\left(101 \mathrm{MHz}, \mathrm{CDCl}_{3}\right)$ spectrum of compound $\mathbf{1 l}$
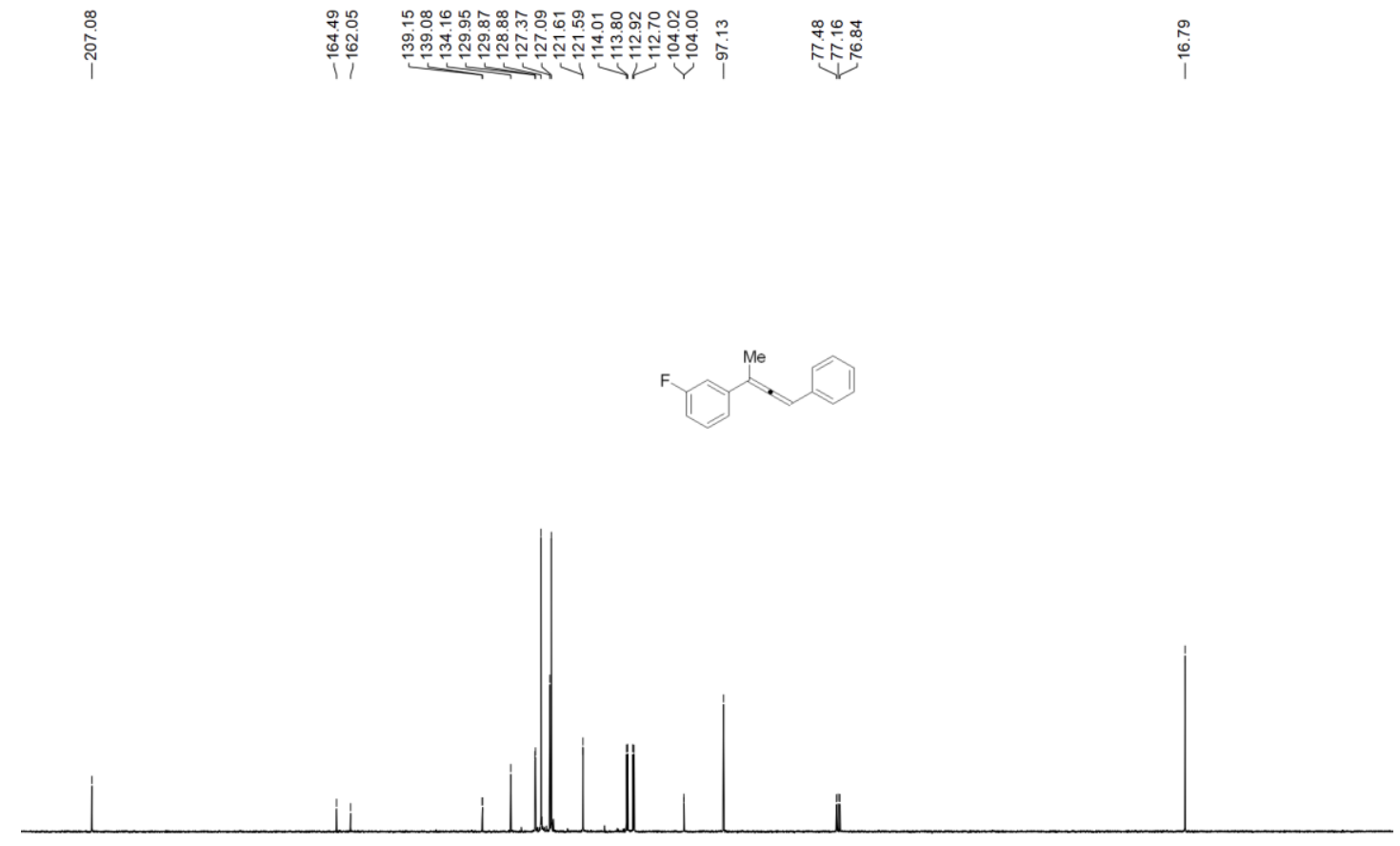

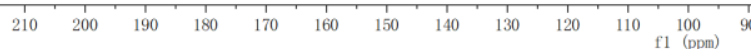


${ }^{1} \mathrm{H}$ NMR (400 MHz, $\left.\mathrm{CDCl}_{3}\right)$ spectrum of compound 10

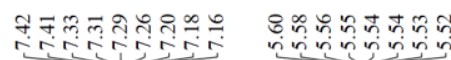

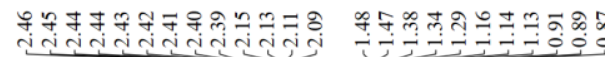

Me
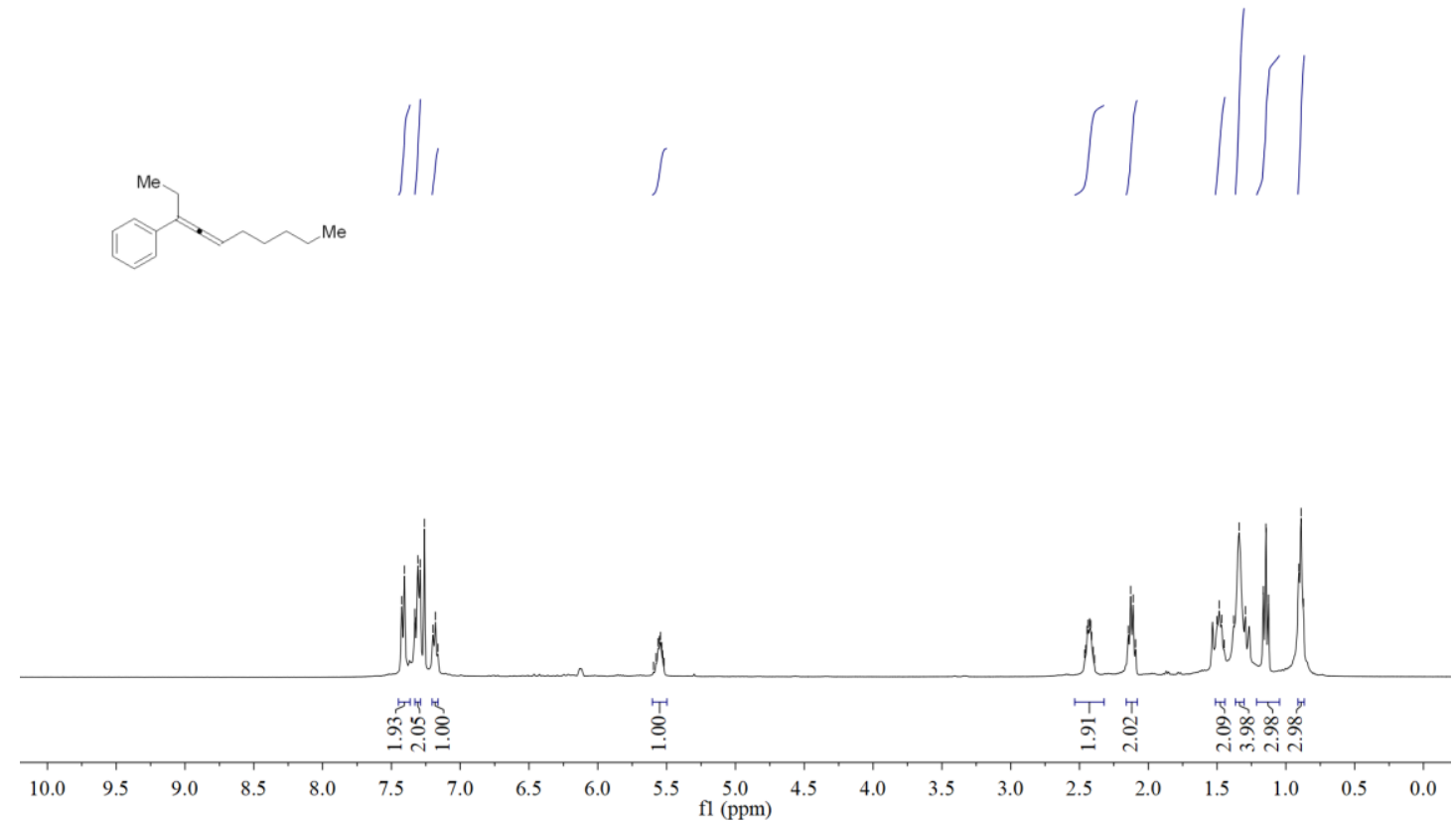

${ }^{13} \mathrm{C} \mathrm{NMR}\left(101 \mathrm{MHz}, \mathrm{CDCl}_{3}\right)$ spectrum of compound $\mathbf{1 0}$

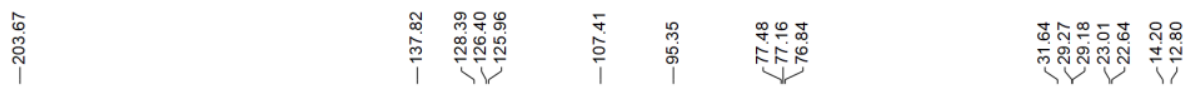

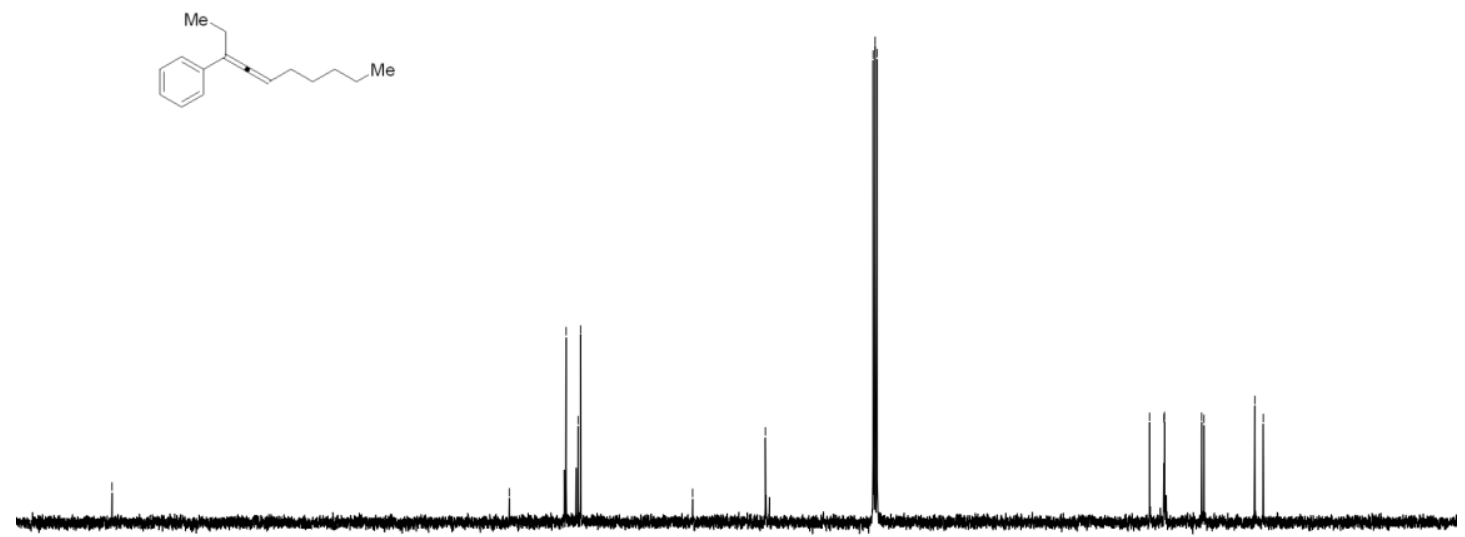


${ }^{1} \mathrm{H} \mathrm{NMR}\left(400 \mathrm{MHz}, \mathrm{CDCl}_{3}\right)$ spectrum of compound 3a

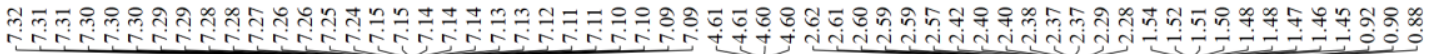
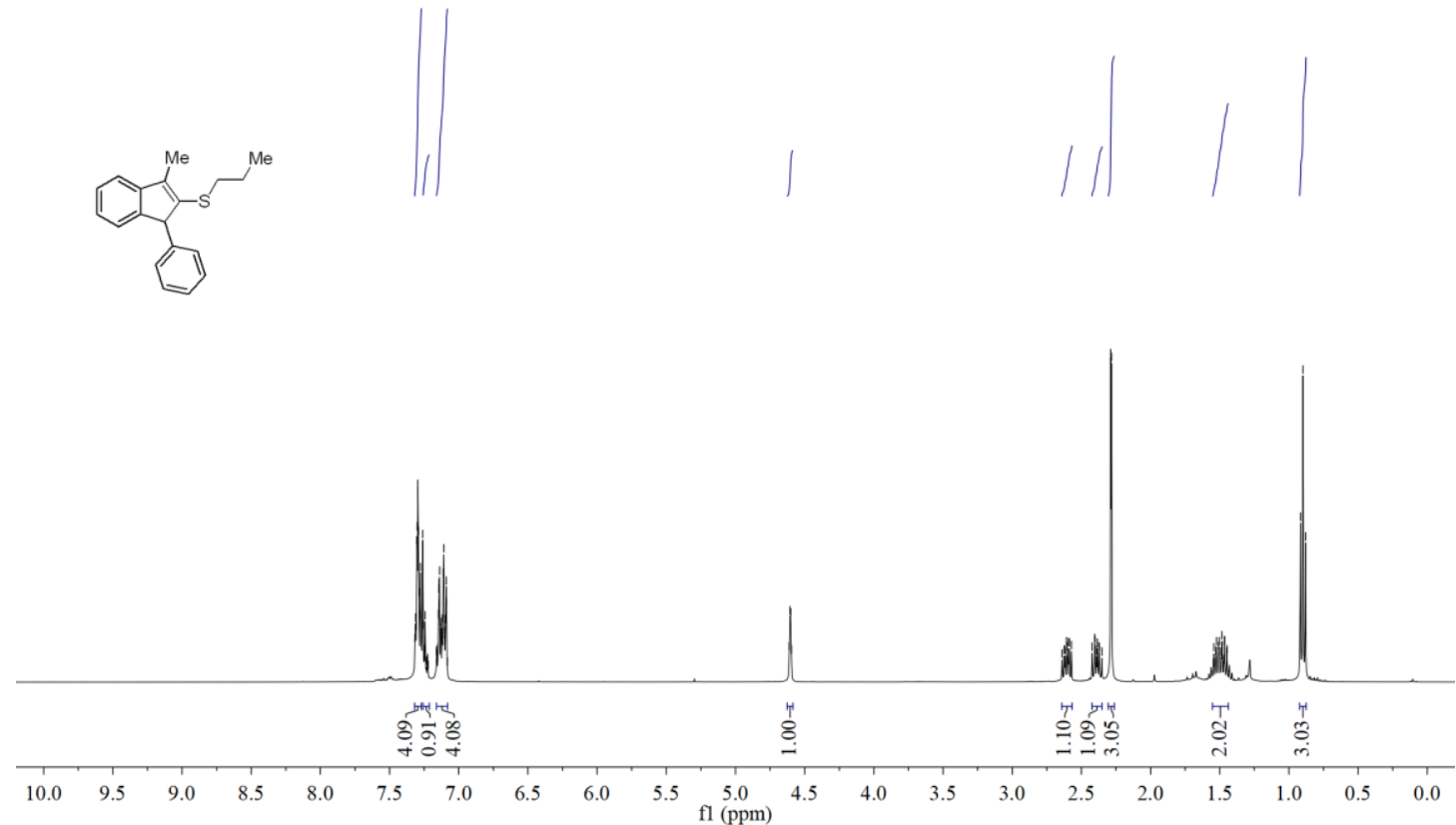

${ }^{13} \mathrm{C} \mathrm{NMR}\left(101 \mathrm{MHz}, \mathrm{CDCl}_{3}\right)$ spectrum of compound 3a

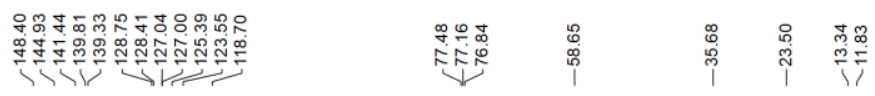
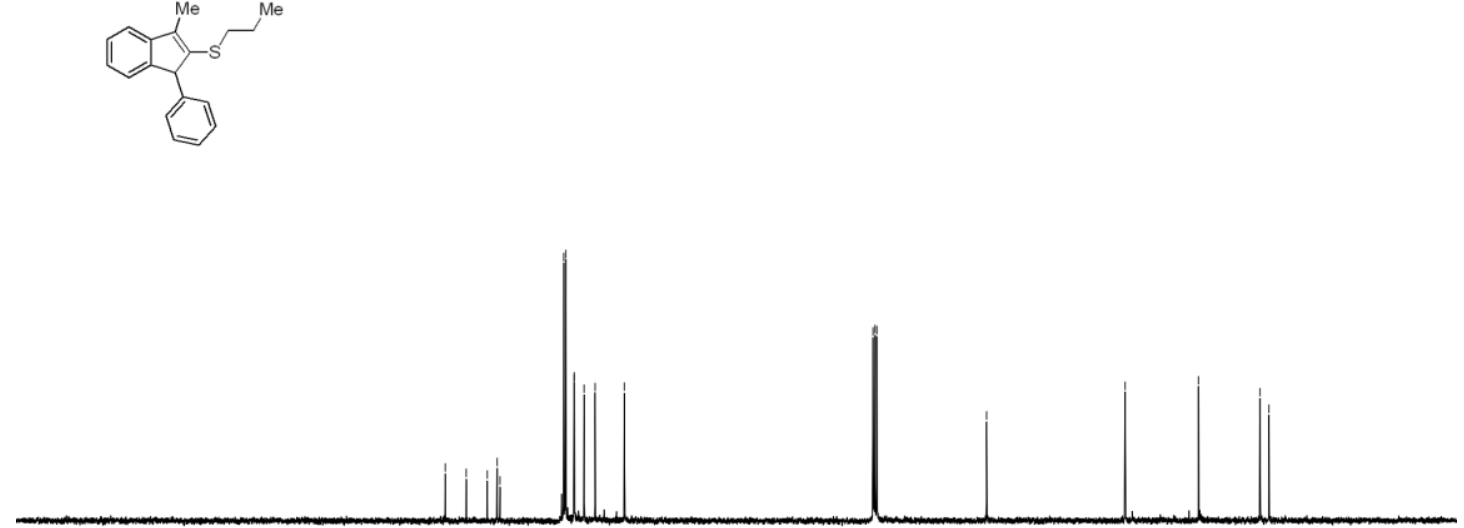
${ }^{1} \mathrm{H}$ NMR (400 MHz, $\left.\mathrm{CDCl}_{3}\right)$ spectrum of compound $\mathbf{3 b}$

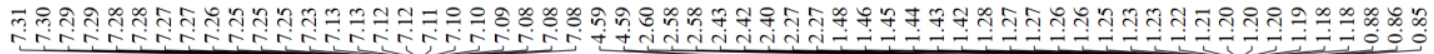
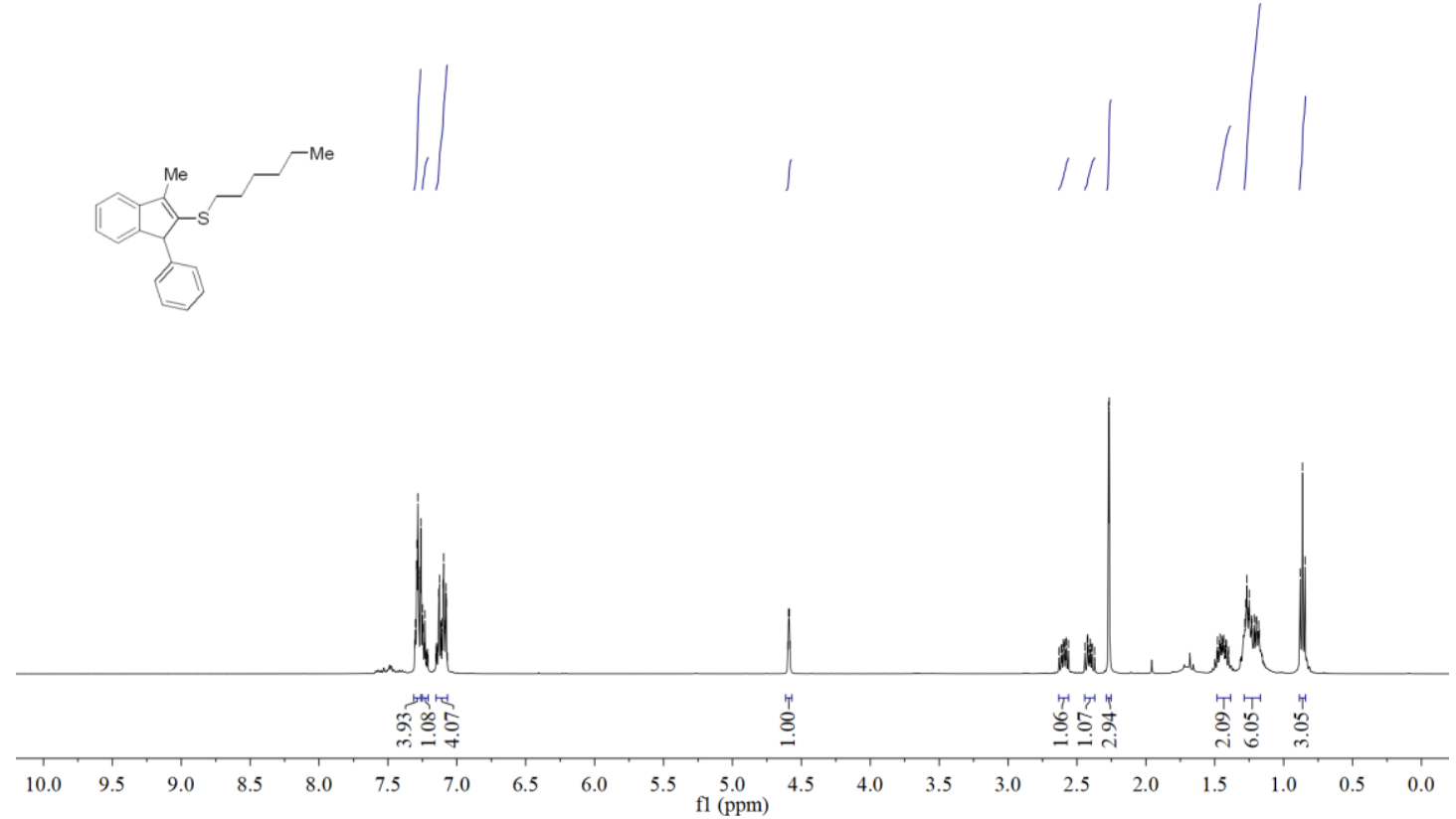

${ }^{13} \mathrm{C} \mathrm{NMR}\left(101 \mathrm{MHz}, \mathrm{CDCl}_{3}\right)$ spectrum of compound $\mathbf{3 b}$

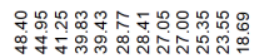

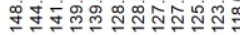
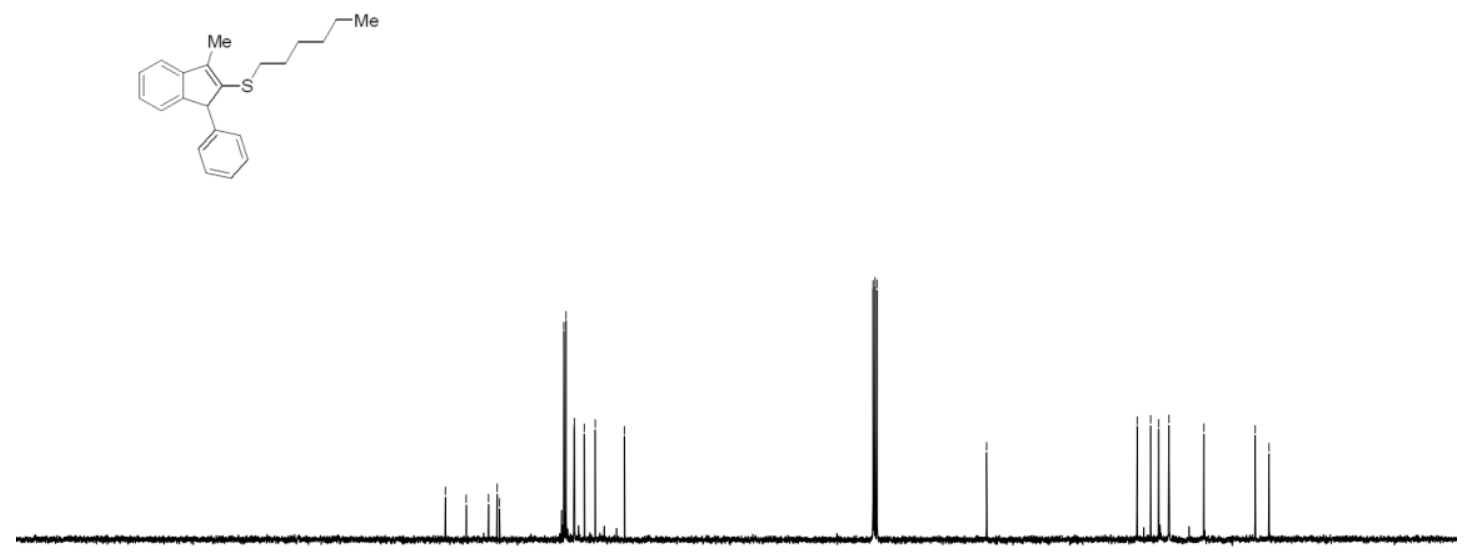
${ }^{1} \mathrm{H}$ NMR $\left(400 \mathrm{MHz}, \mathrm{CDCl}_{3}\right)$ spectrum of compound $\mathbf{3 c}$

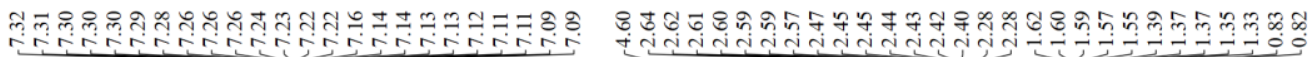
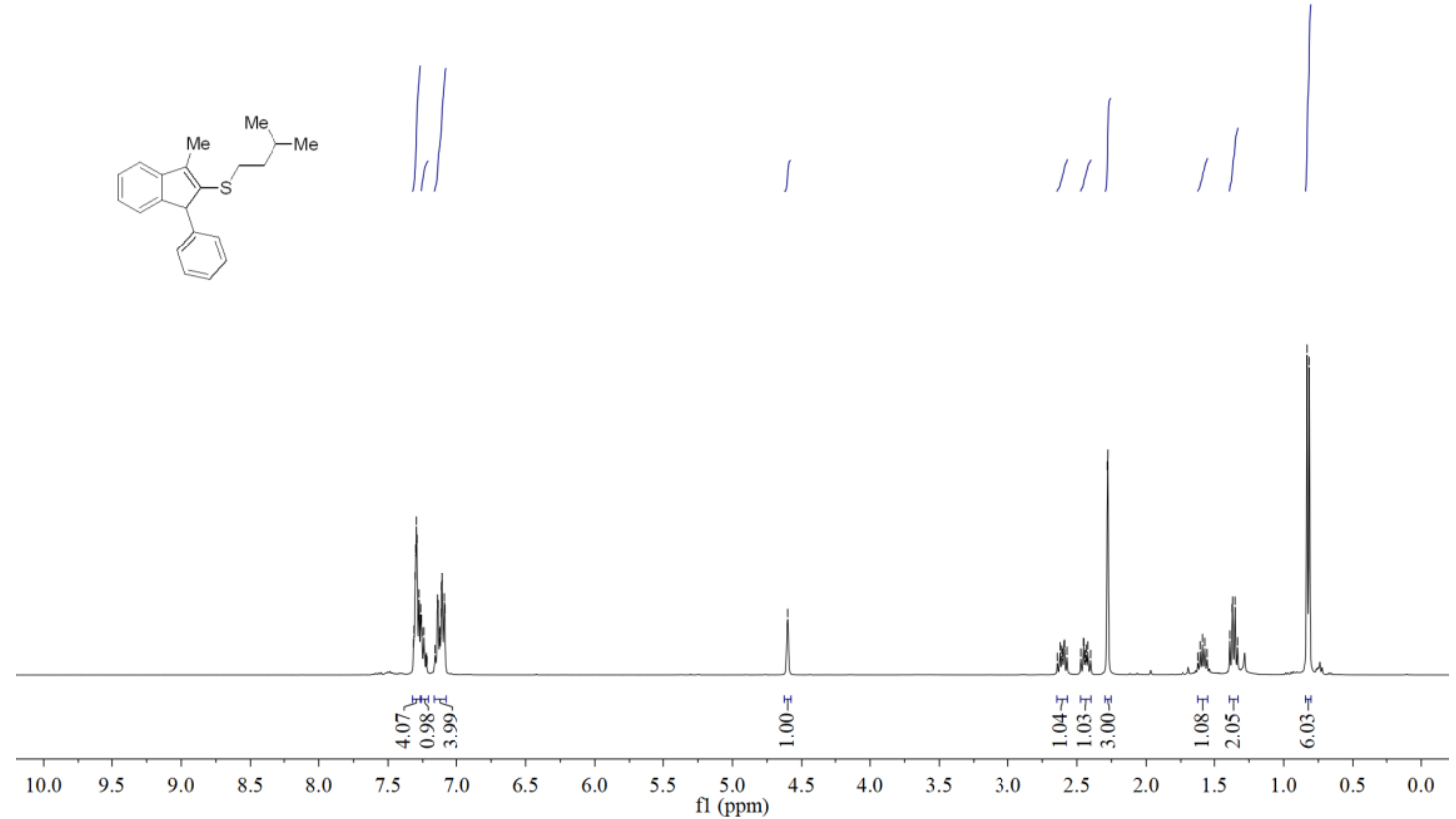

${ }^{13} \mathrm{C} \mathrm{NMR}\left(101 \mathrm{MHz}, \mathrm{CDCl}_{3}\right)$ spectrum of compound $\mathbf{3 c}$

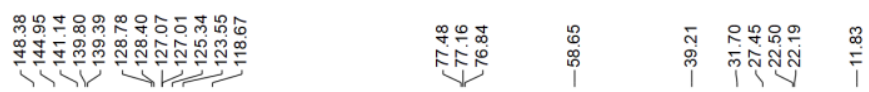

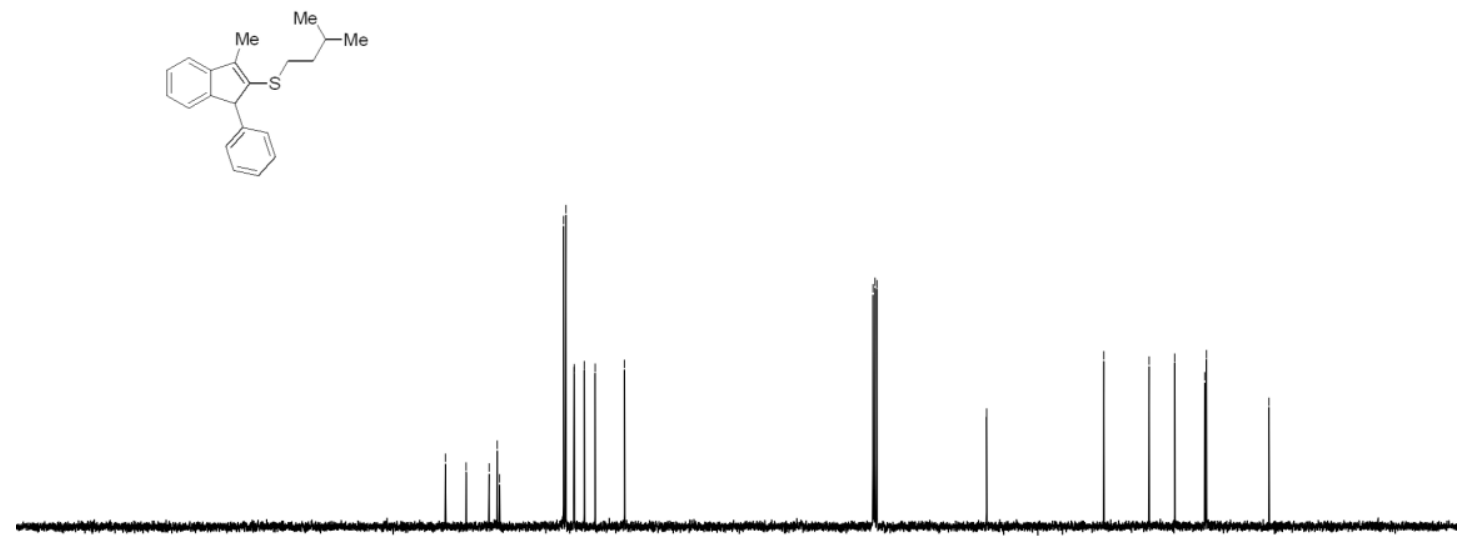

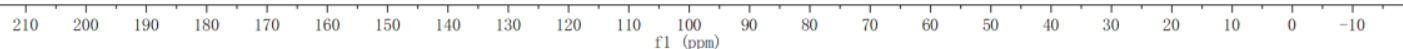


${ }^{1} \mathrm{H}$ NMR (400 MHz, $\mathrm{CDCl}_{3}$ ) spectrum of compound $\mathbf{3 d}$

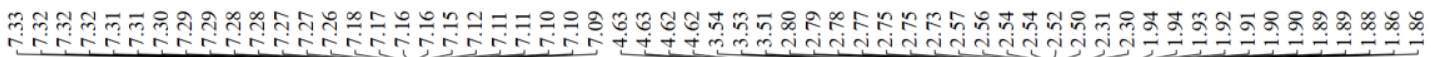
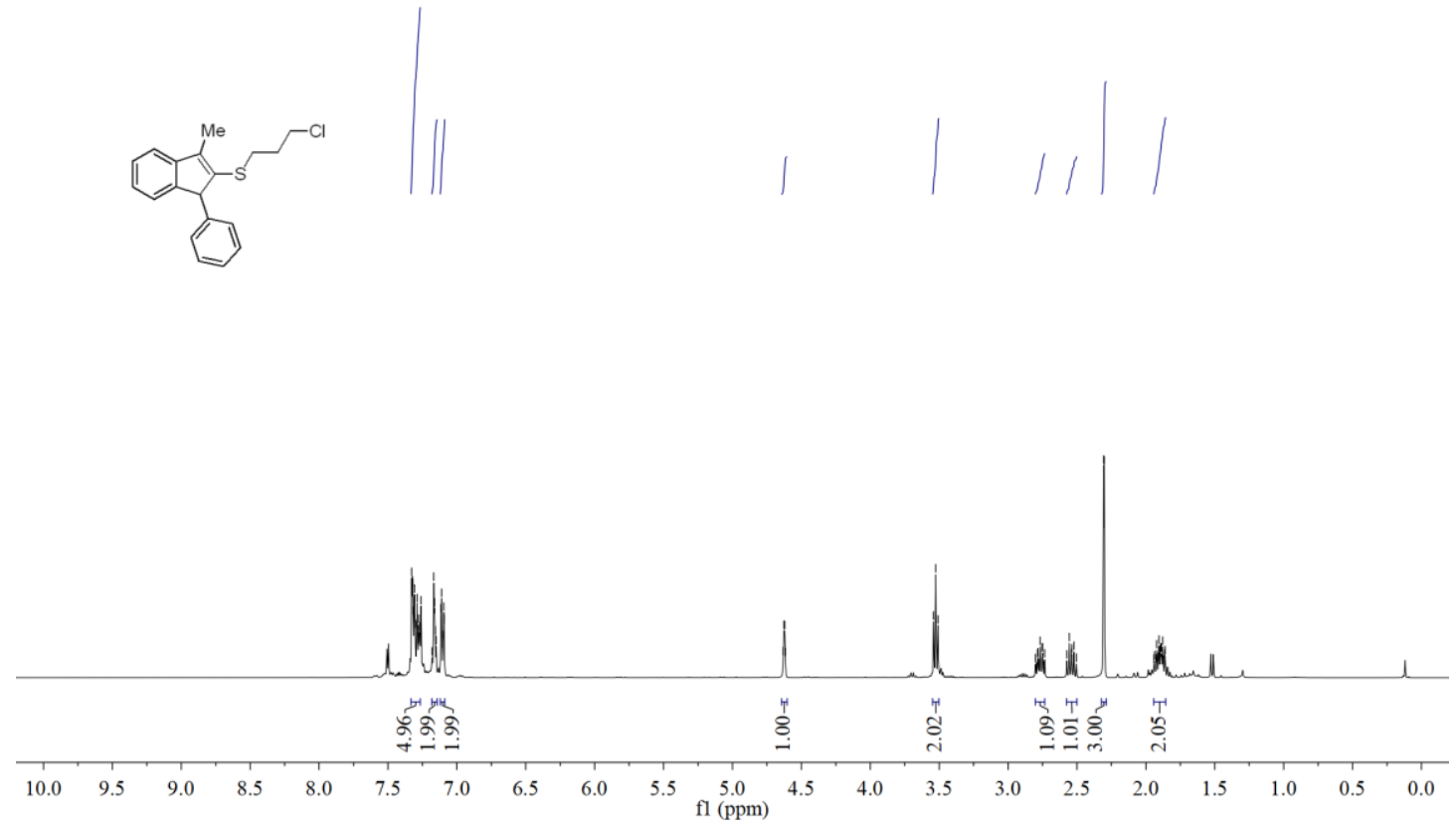

${ }^{13} \mathrm{C} \mathrm{NMR}\left(101 \mathrm{MHz}, \mathrm{CDCl}_{3}\right)$ spectrum of compound $\mathbf{3 d}$

\begin{tabular}{|c|}
\hline 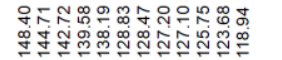 \\
\hline
\end{tabular}
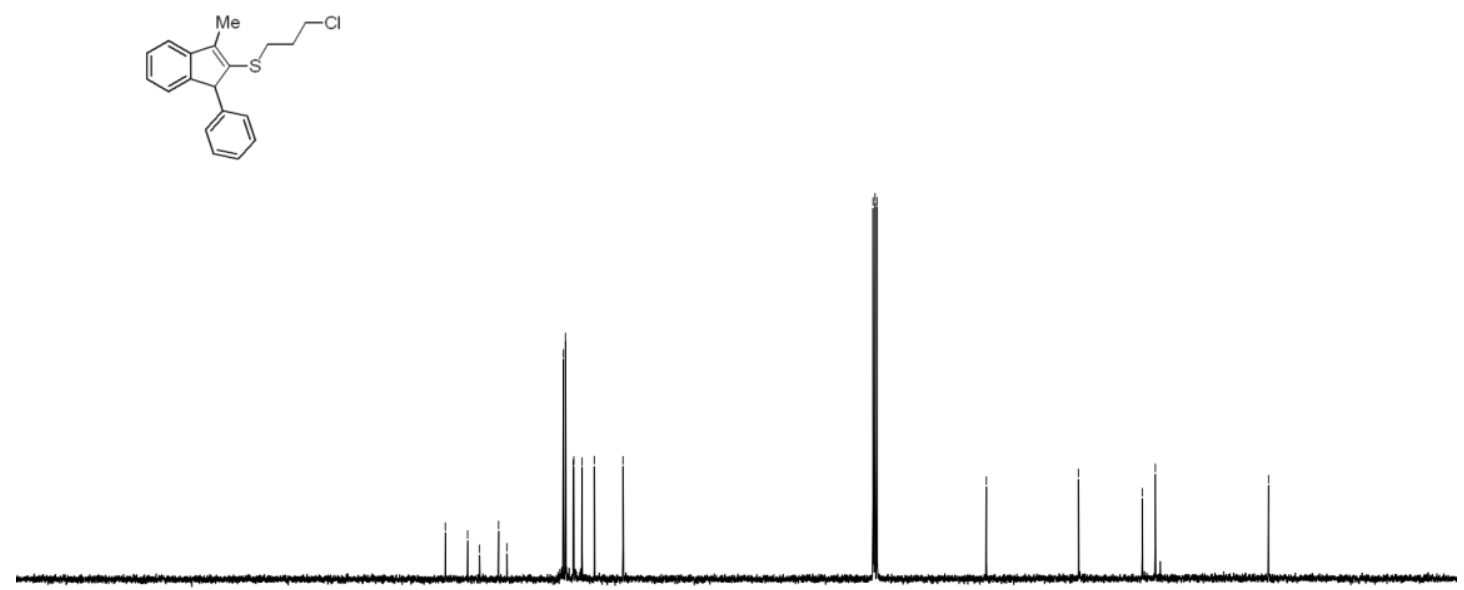

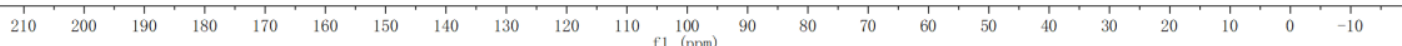


${ }^{1} \mathrm{H} \mathrm{NMR}\left(400 \mathrm{MHz}, \mathrm{CDCl}_{3}\right)$ spectrum of compound $\mathbf{3 e}$

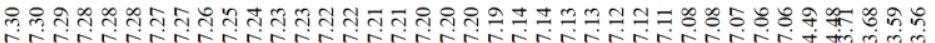

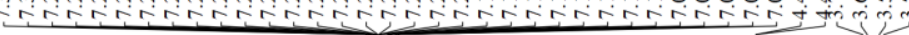
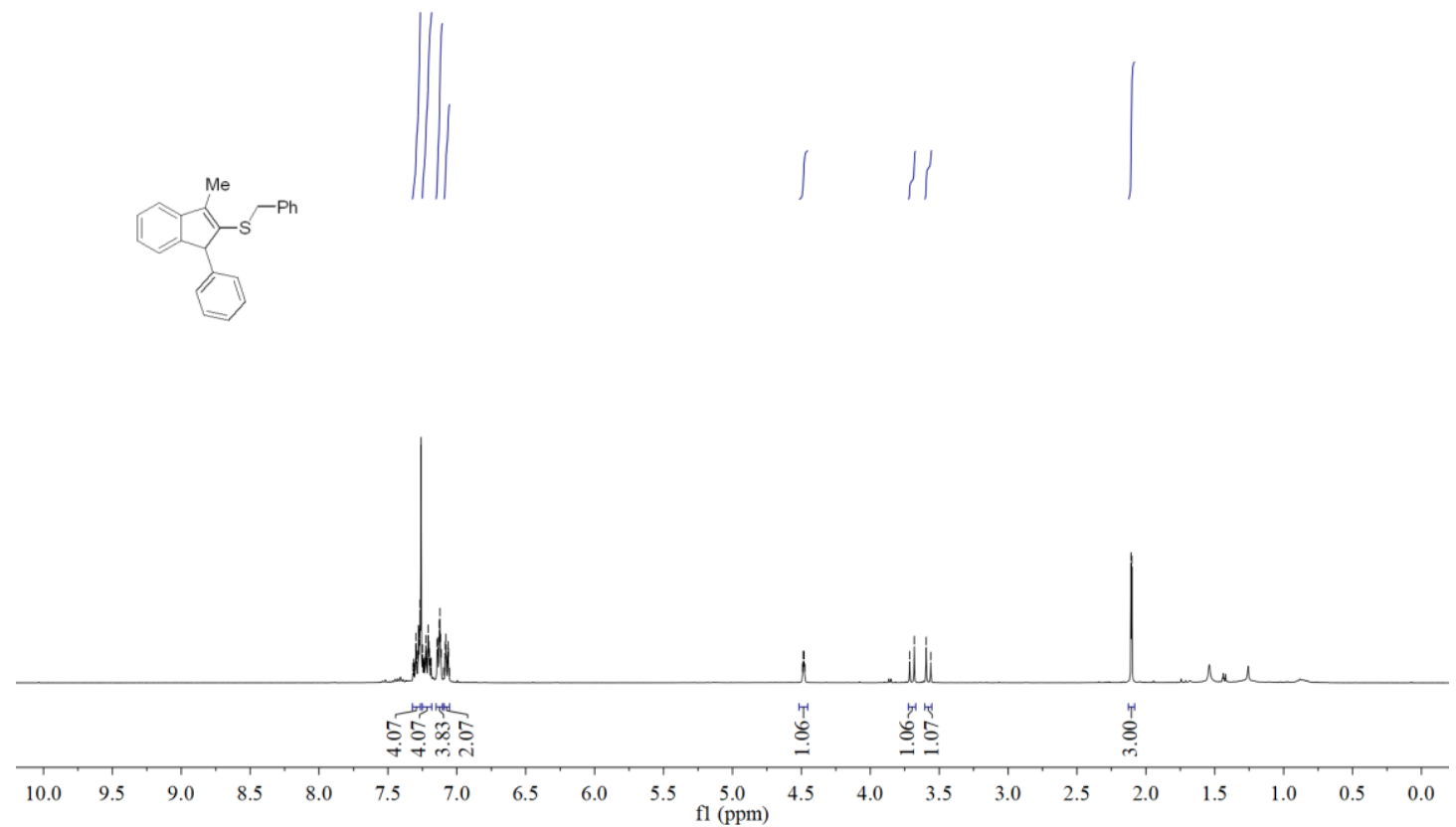

${ }^{13} \mathrm{C} \mathrm{NMR}\left(101 \mathrm{MHz}, \mathrm{CDCl}_{3}\right)$ spectrum of compound $\mathbf{3 e}$

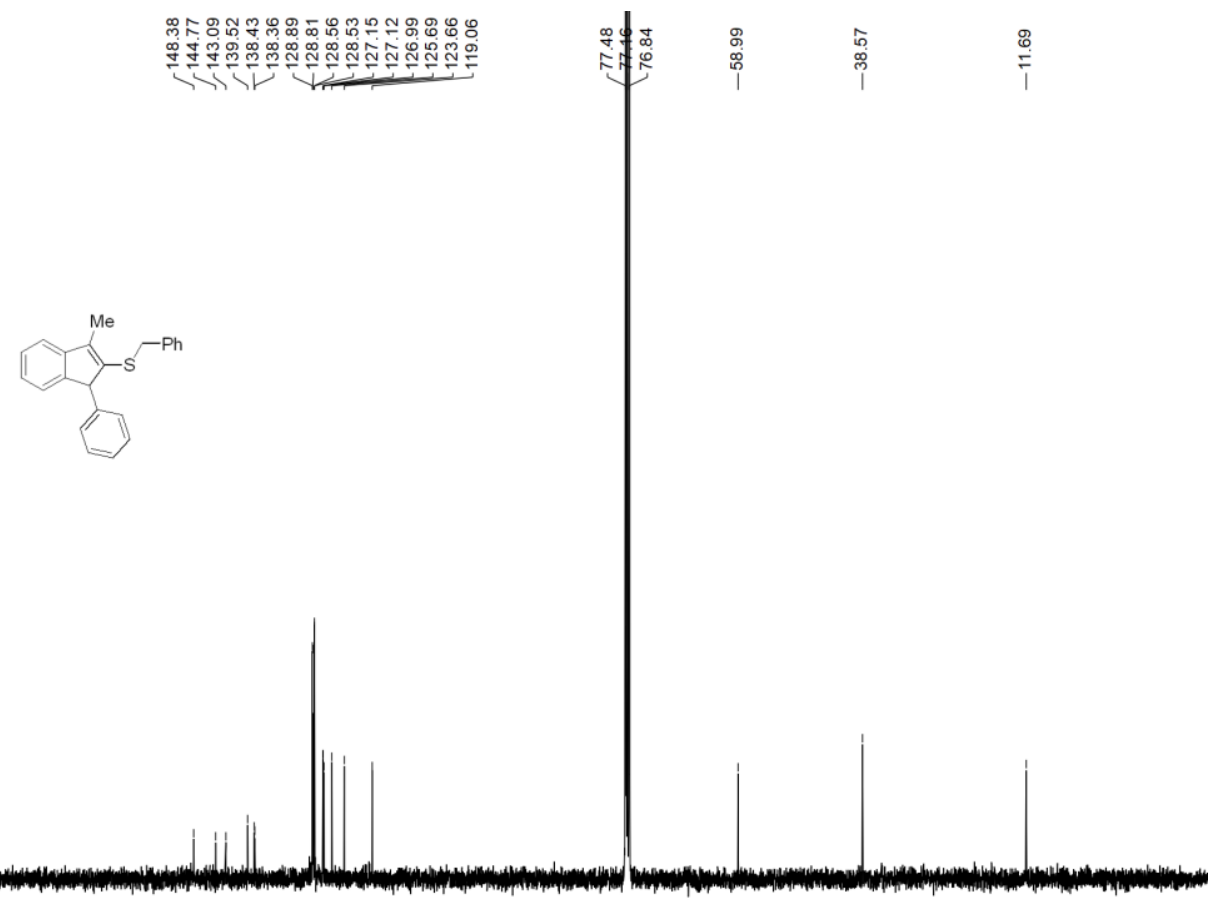

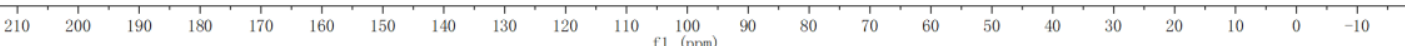


${ }^{1} \mathrm{H}$ NMR (400 MHz, $\left.\mathrm{CDCl}_{3}\right)$ spectrum of compound $\mathbf{3 f}$

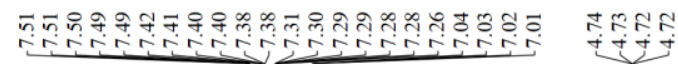
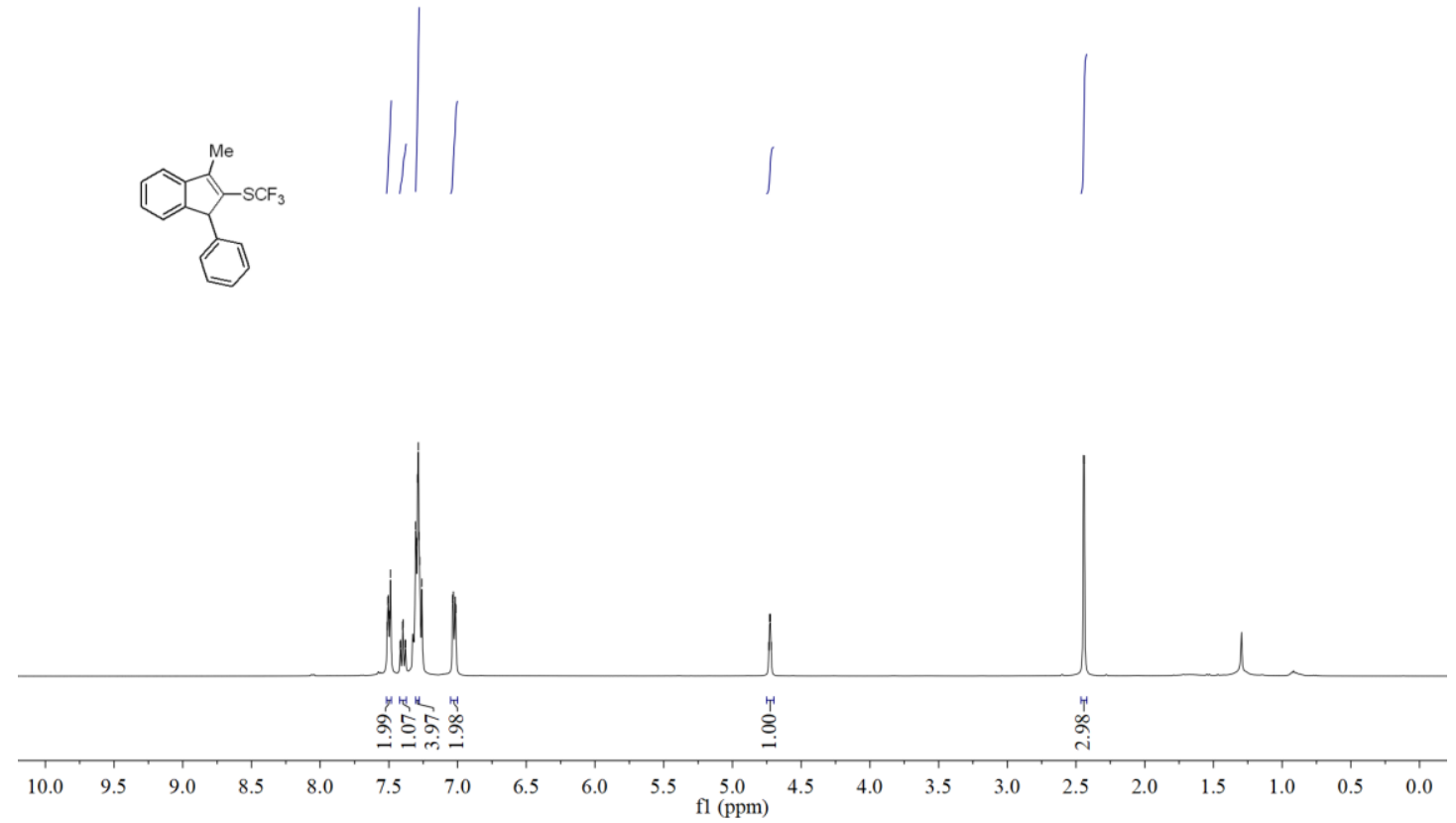

${ }^{13} \mathrm{C} \mathrm{NMR}\left(101 \mathrm{MHz}, \mathrm{CDCl}_{3}\right)$ spectrum of compound $\mathbf{3 f}$

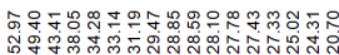

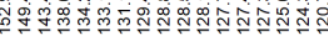
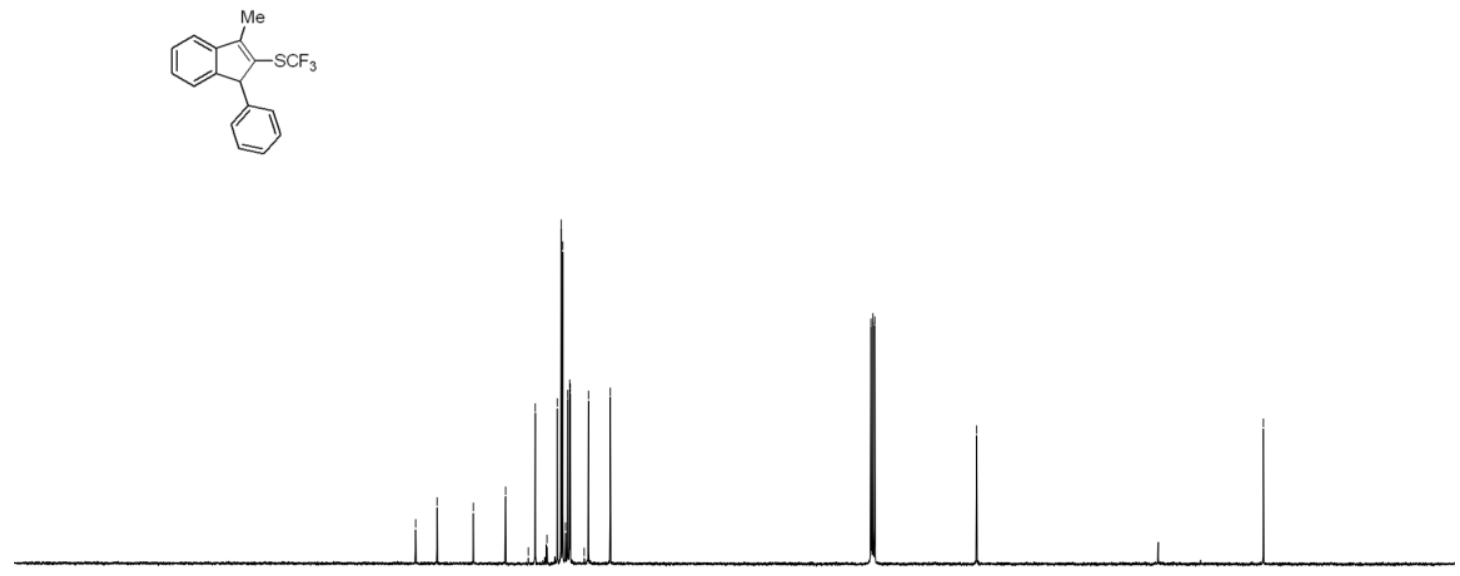

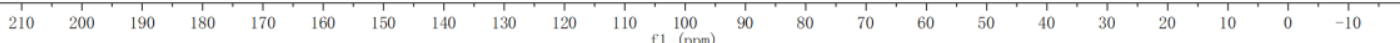


${ }^{1} \mathrm{H} \mathrm{NMR}\left(400 \mathrm{MHz}, \mathrm{CDCl}_{3}\right.$ ) spectrum of compound $\mathbf{3 g}$

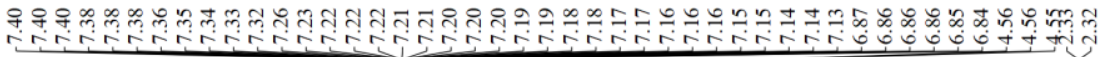

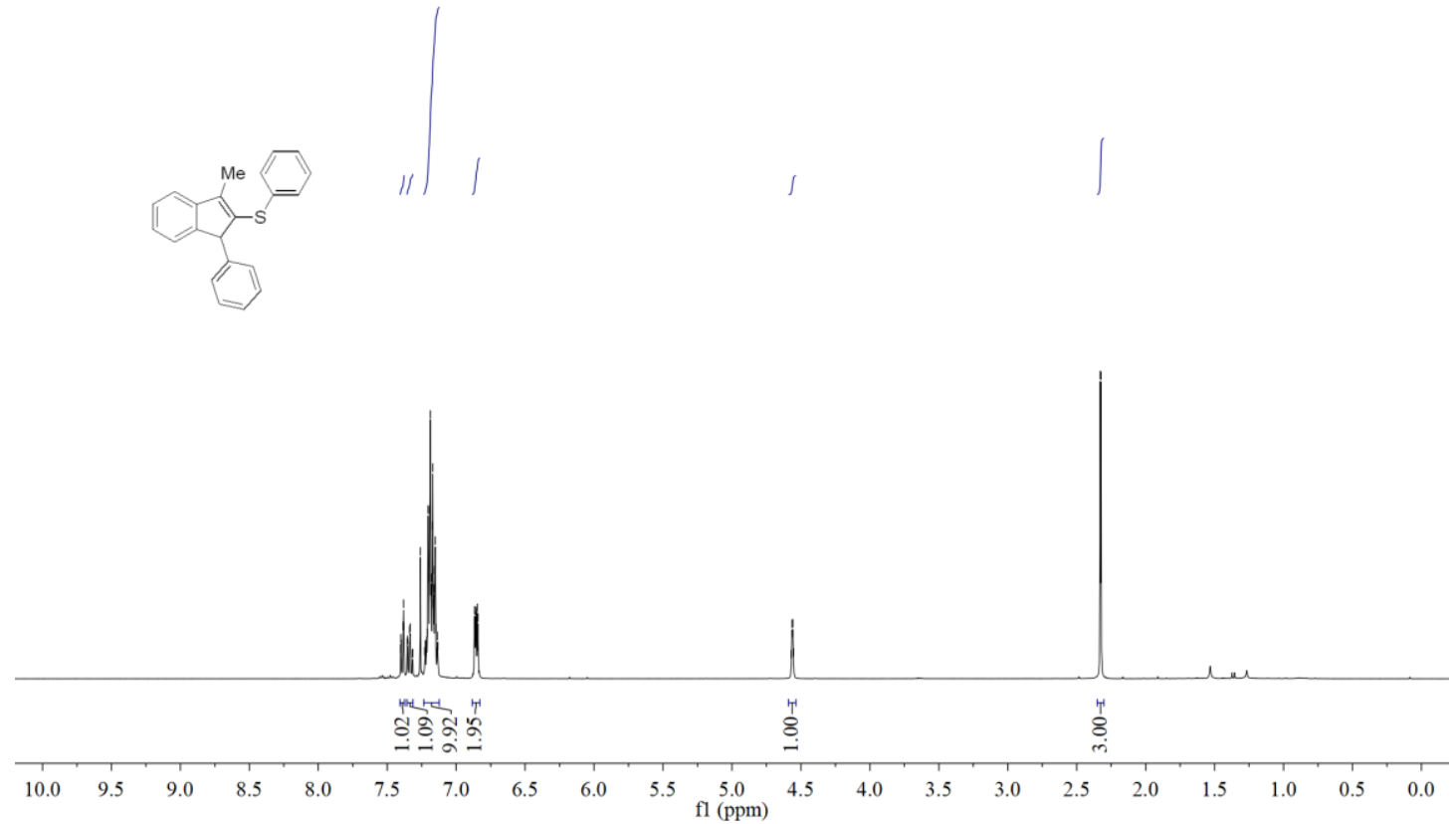

${ }^{13} \mathrm{C} \mathrm{NMR}\left(101 \mathrm{MHz}, \mathrm{CDCl}_{3}\right)$ spectrum of compound $\mathbf{3 g}$

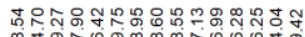

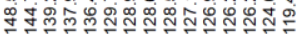

尔尔

ํํํ
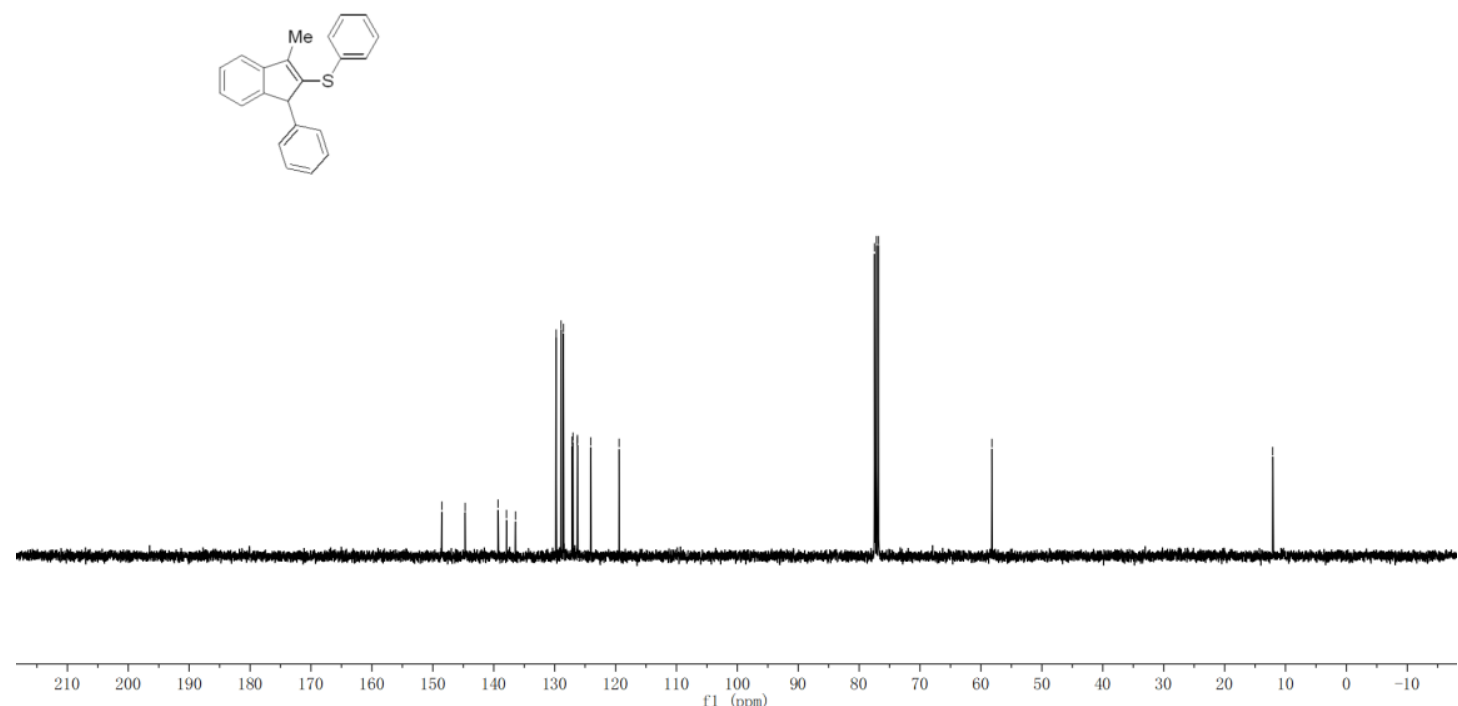
${ }^{1} \mathrm{H}$ NMR (400 MHz, $\mathrm{CDCl}_{3}$ ) spectrum of compound $\mathbf{3 h}$

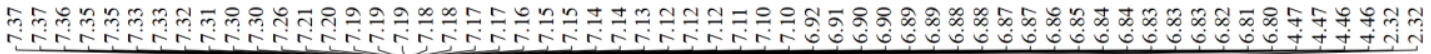

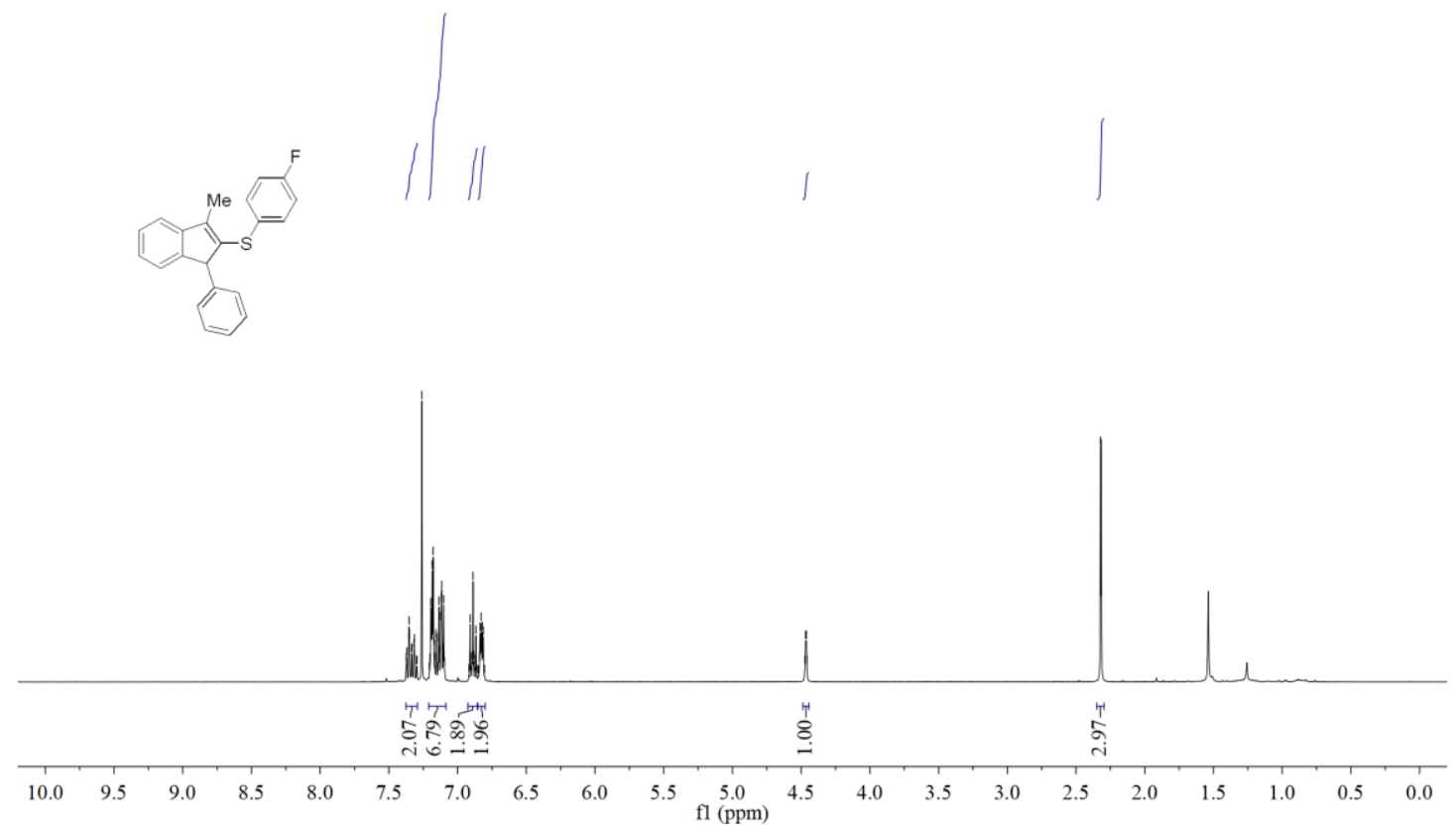

${ }^{13} \mathrm{C} \mathrm{NMR}\left(101 \mathrm{MHz}, \mathrm{CDCl}_{3}\right)$ spectrum of compound $\mathbf{3 h}$

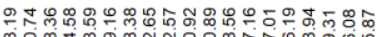

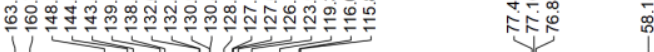

$\stackrel{\text { : }}{\leftarrow}$

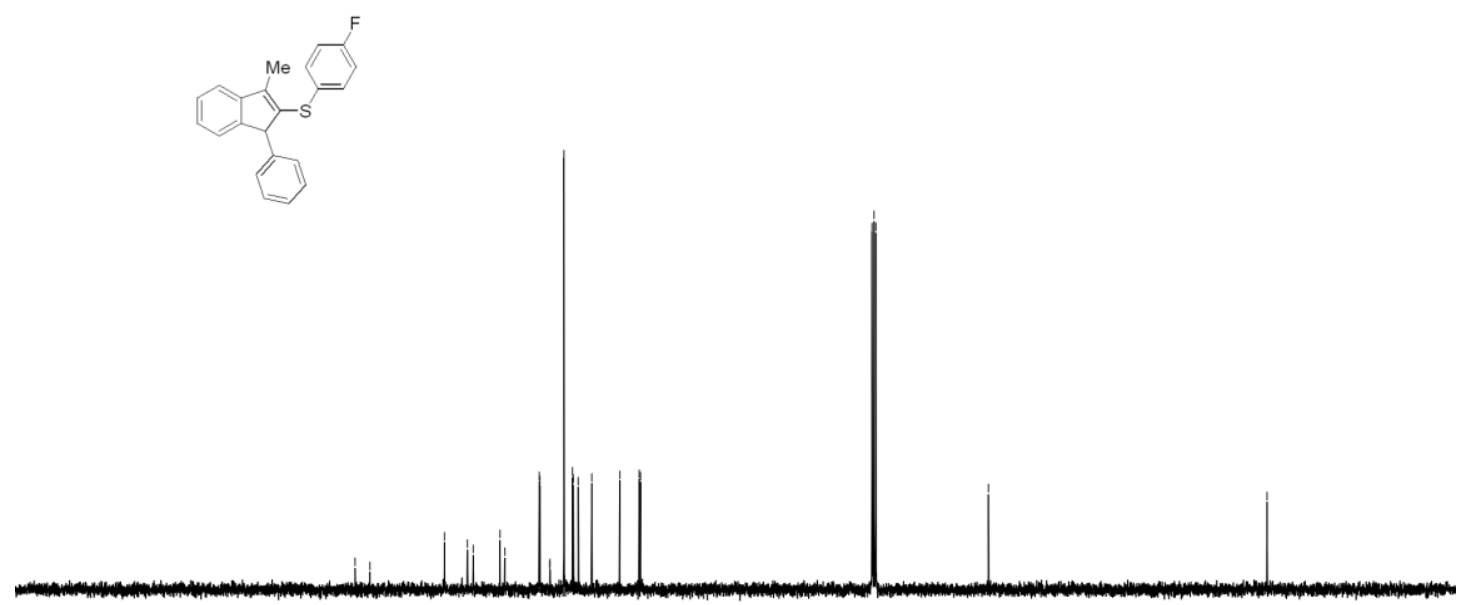

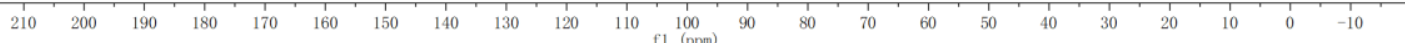


${ }^{1} \mathrm{H} \mathrm{NMR}\left(400 \mathrm{MHz}, \mathrm{CDCl}_{3}\right.$ ) spectrum of compound 3i

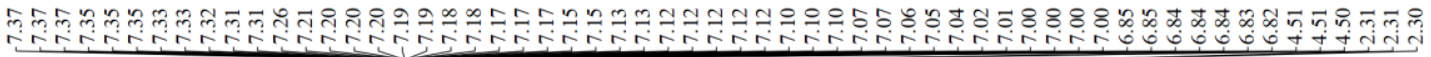

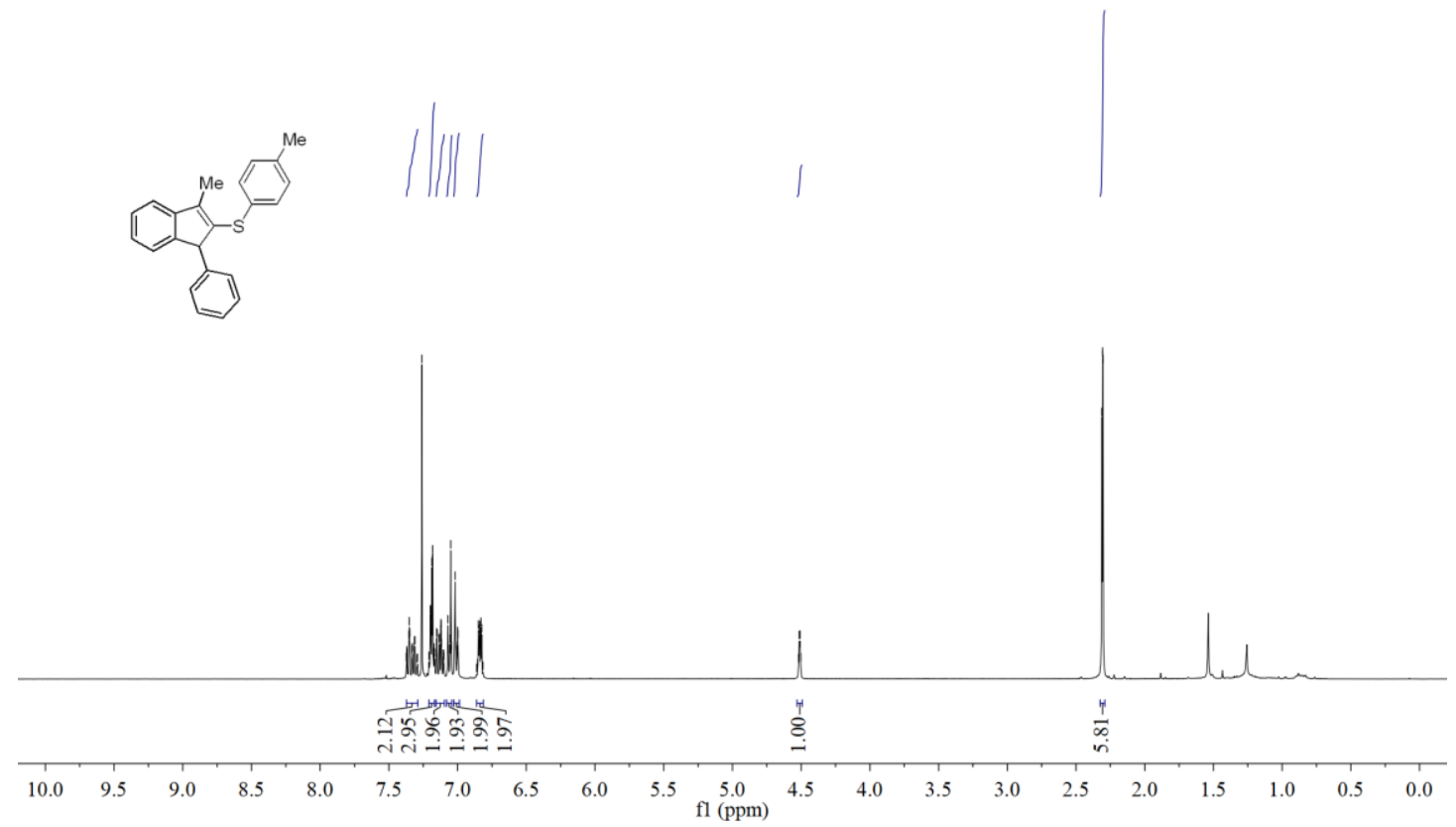

${ }^{13} \mathrm{C} \mathrm{NMR}\left(101 \mathrm{MHz}, \mathrm{CDCl}_{3}\right)$ spectrum of compound $3 \mathbf{i}$

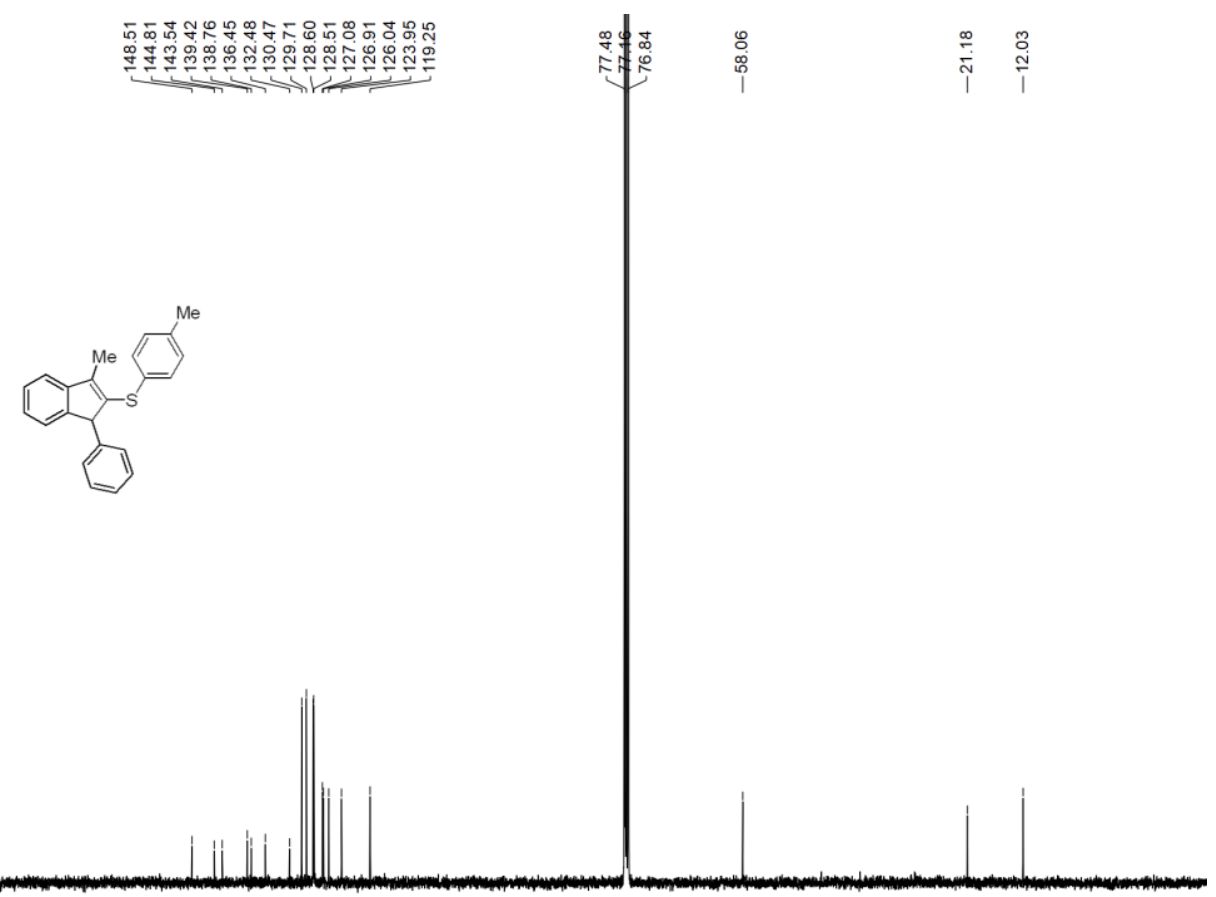

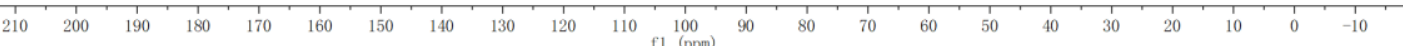


${ }^{1} \mathrm{H} \mathrm{NMR}\left(400 \mathrm{MHz}, \mathrm{CDCl}_{3}\right.$ ) spectrum of compound $\mathbf{3 j}$

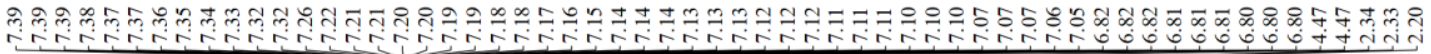

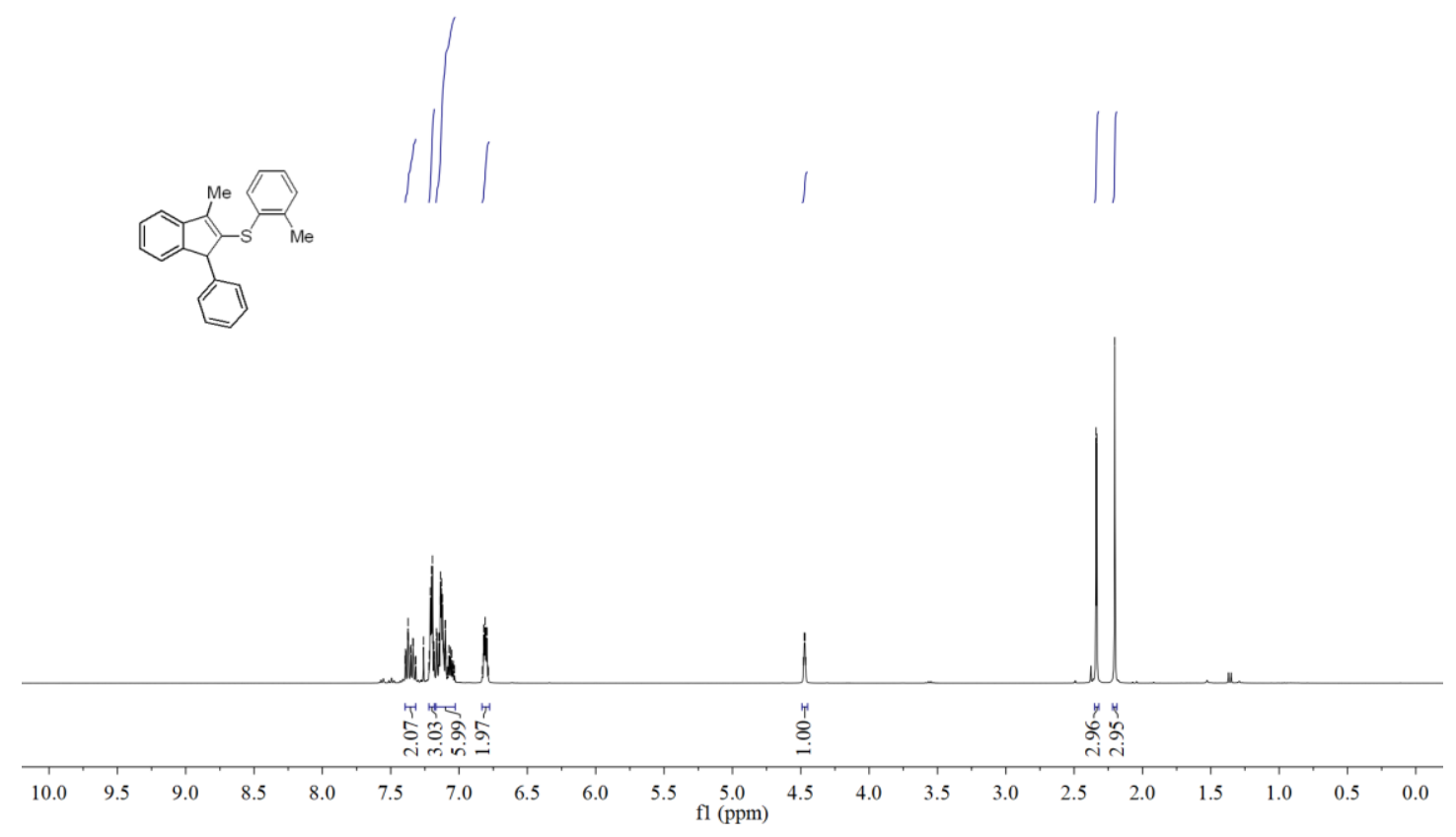

${ }^{13} \mathrm{C} \mathrm{NMR}\left(101 \mathrm{MHz}, \mathrm{CDCl}_{3}\right)$ spectrum of compound $\mathbf{3 j}$

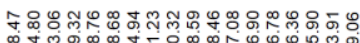

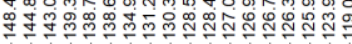

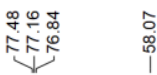

กั

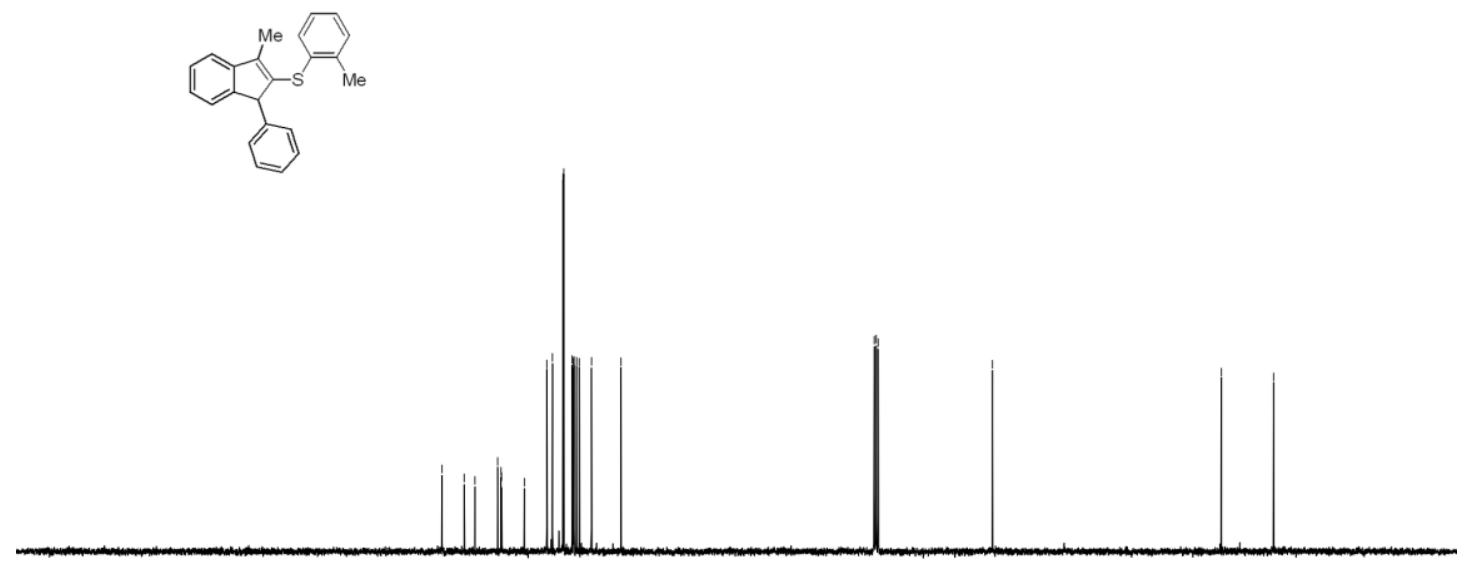


${ }^{1} \mathrm{H} \mathrm{NMR}\left(400 \mathrm{MHz}, \mathrm{CDCl}_{3}\right)$ spectrum of compound $\mathbf{3 k}$

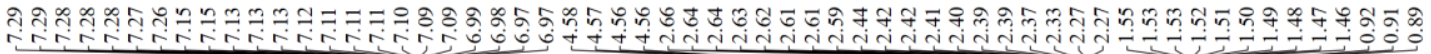

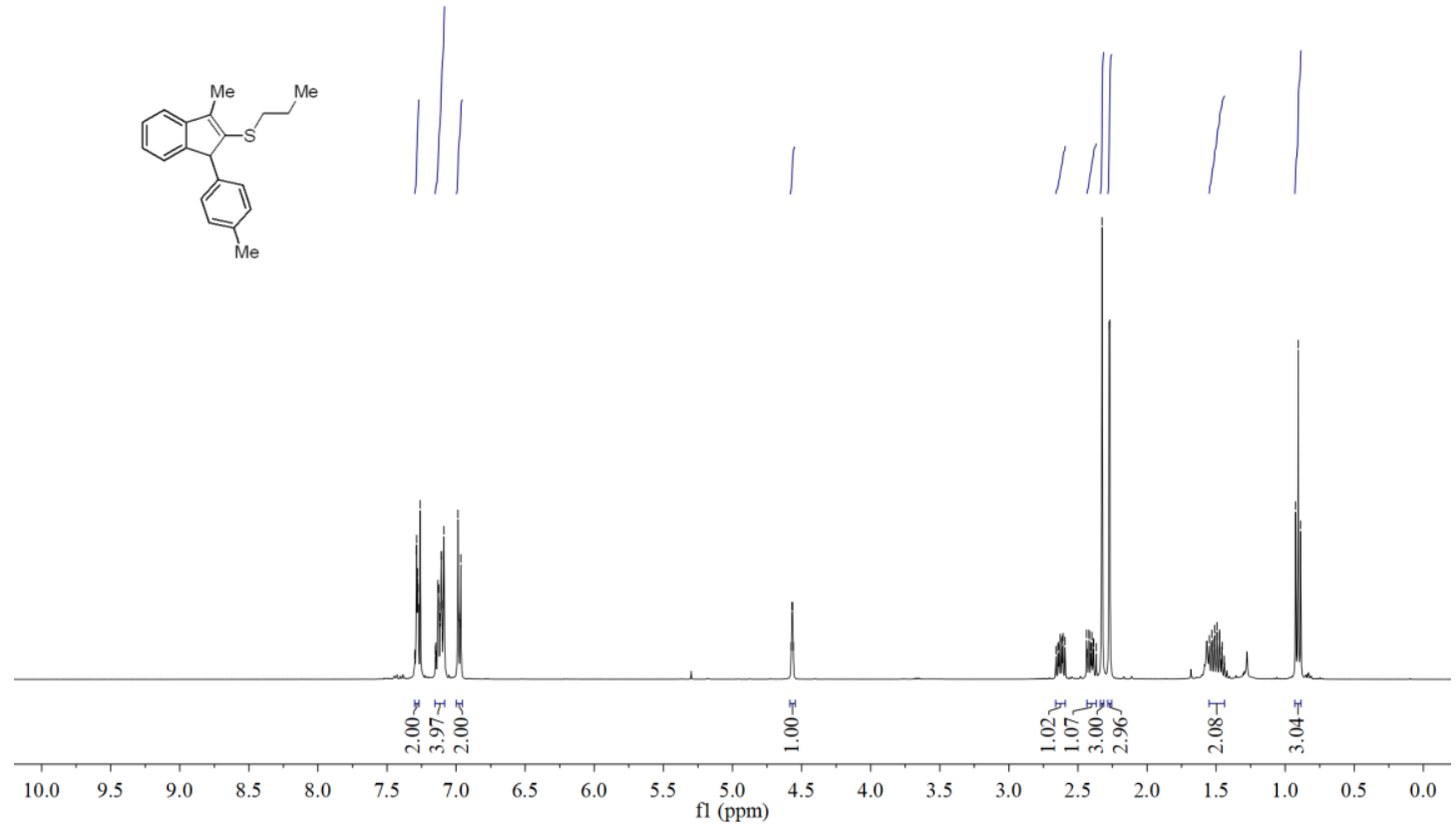

${ }^{13} \mathrm{C} \mathrm{NMR}\left(101 \mathrm{MHz}, \mathrm{CDCl}_{3}\right)$ spectrum of compound $\mathbf{3 k}$
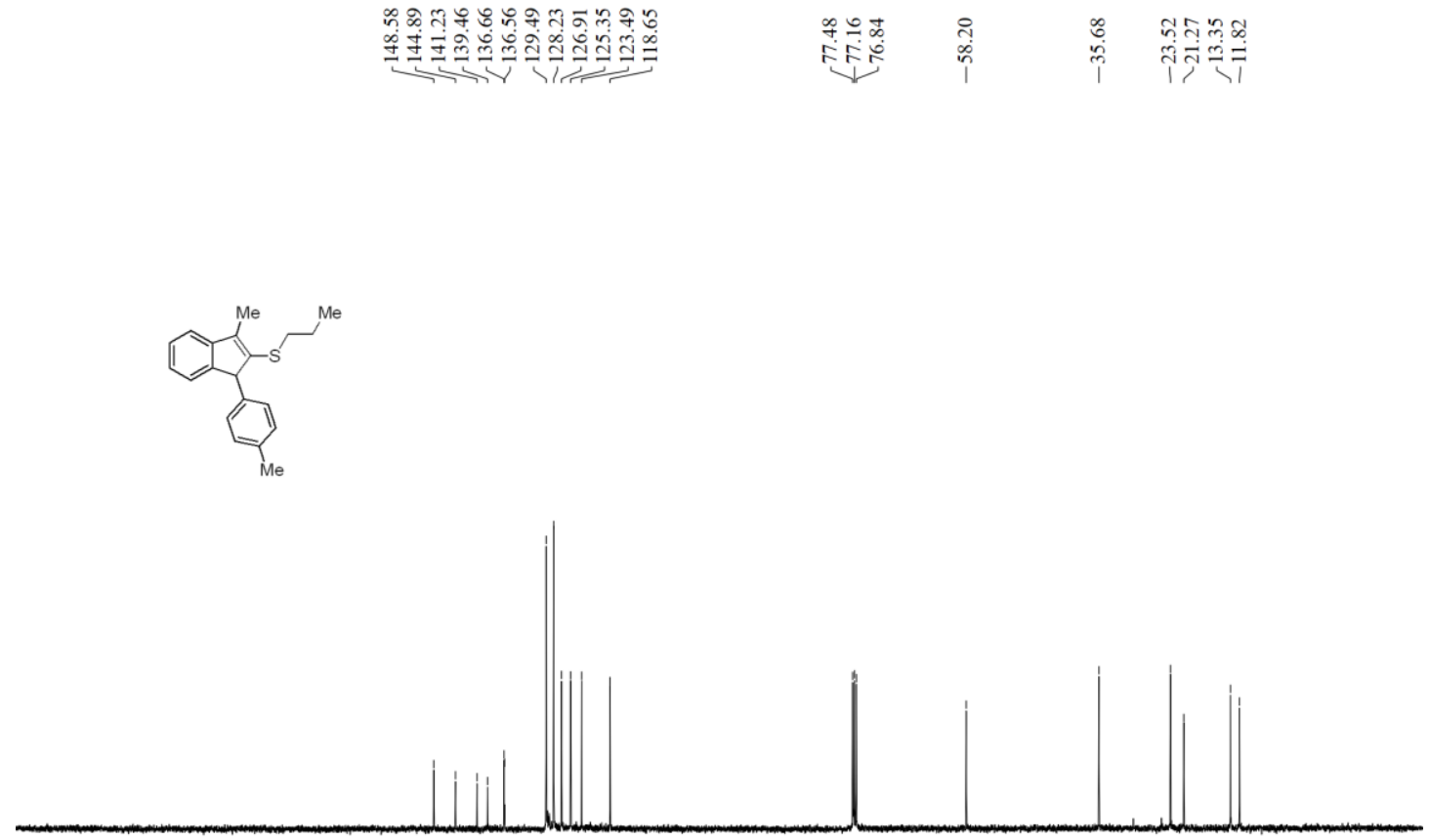

$\begin{array}{lllllllllllllllllllllll}210 & 200 & 190 & 180 & 170 & 160 & 150 & 140 & 130 & 120 & 110 & 100 & 90 & 80 & 70 & 60 & 50 & 40 & 30 & 20 & 10 & 0 & -10\end{array}$ 
${ }^{1} \mathrm{H}$ NMR (400 MHz, $\mathrm{CDCl}_{3}$ ) spectrum of compound $3 \mathbf{l}$

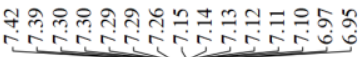

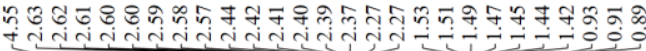
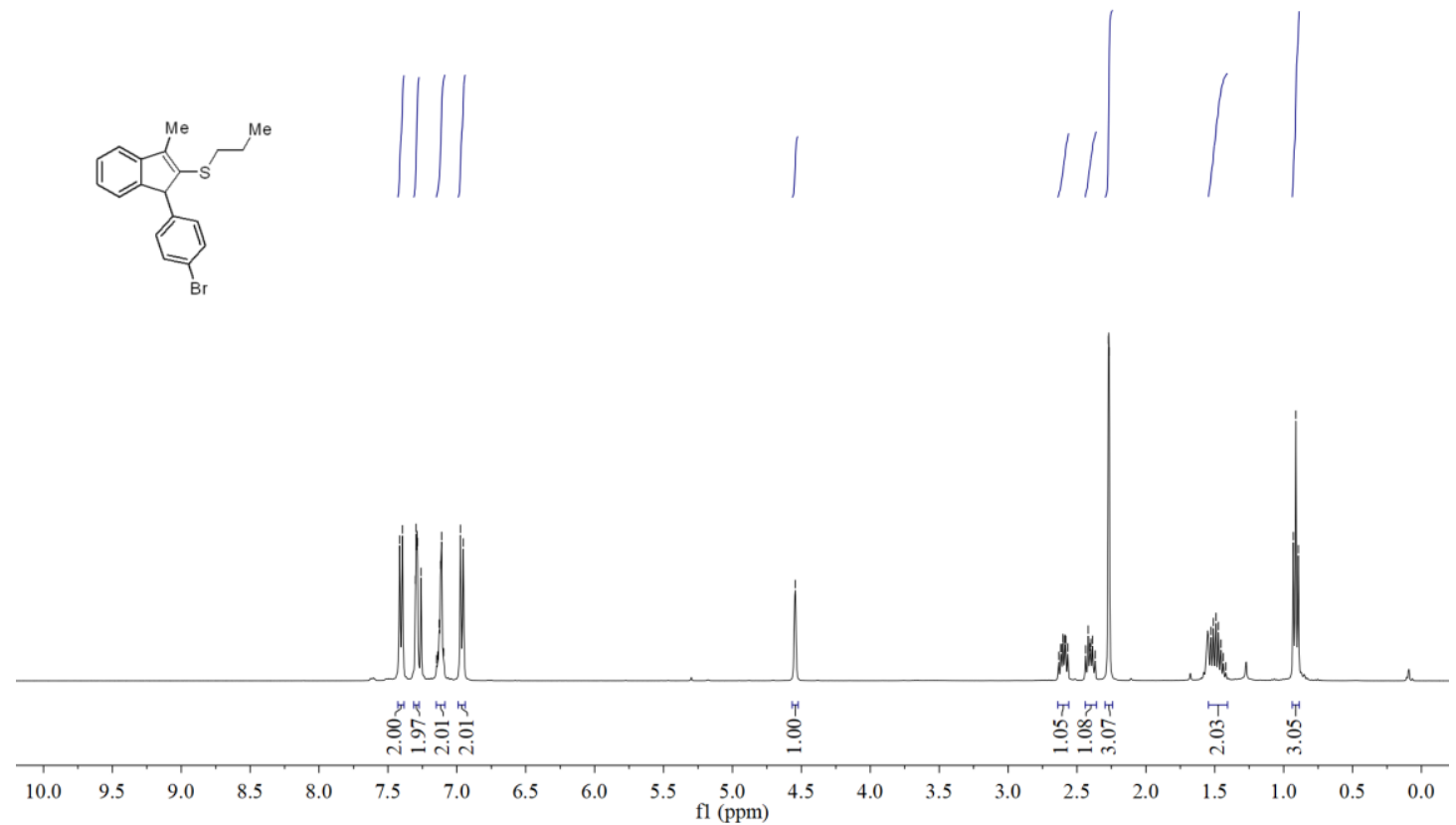

${ }^{13} \mathrm{C} \mathrm{NMR}\left(101 \mathrm{MHz}, \mathrm{CDCl}_{3}\right)$ spectrum of compound $3 \mathbf{I}$

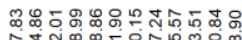

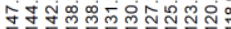

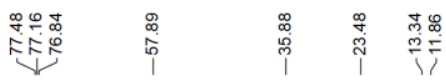
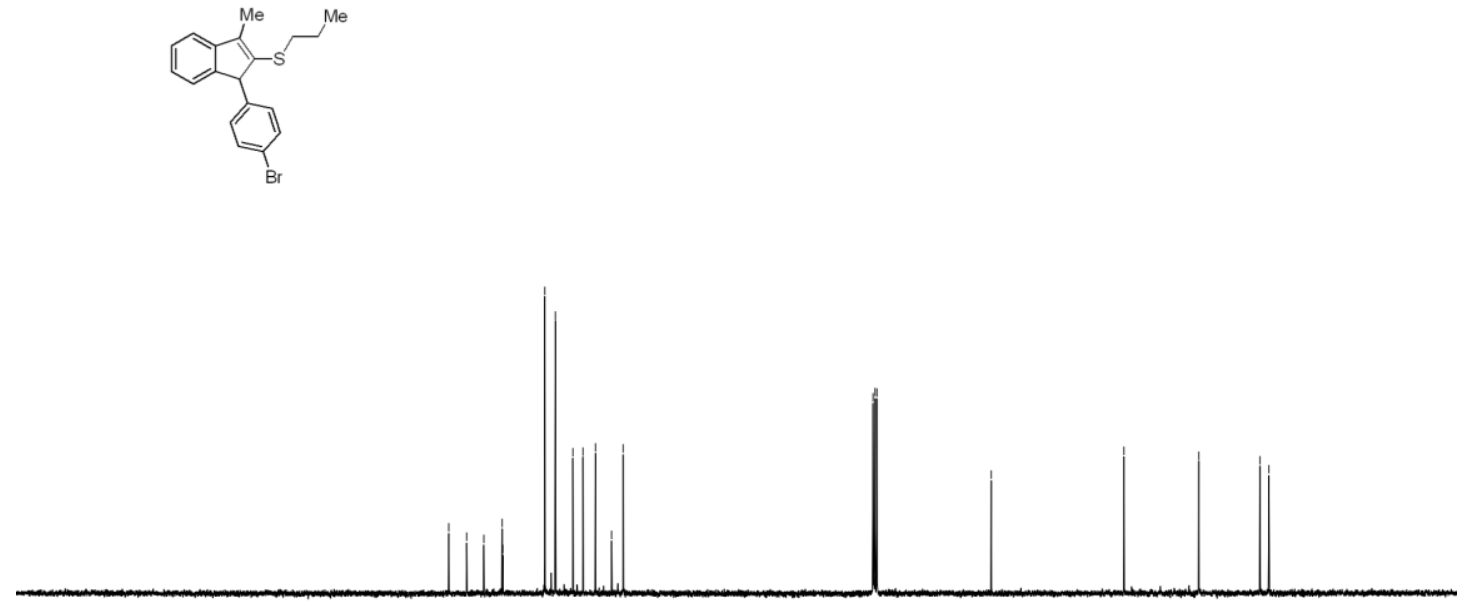

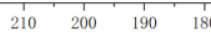
$170 \quad 160 \quad 150$ ${ }_{140}^{1} \quad 130$ $110 \stackrel{100}{100}$ $80 \quad 70$ 
${ }^{1} \mathrm{H}$ NMR (400 MHz, $\mathrm{CDCl}_{3}$ ) spectrum of compound 3m

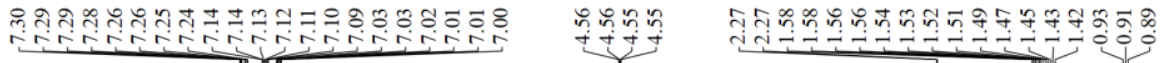
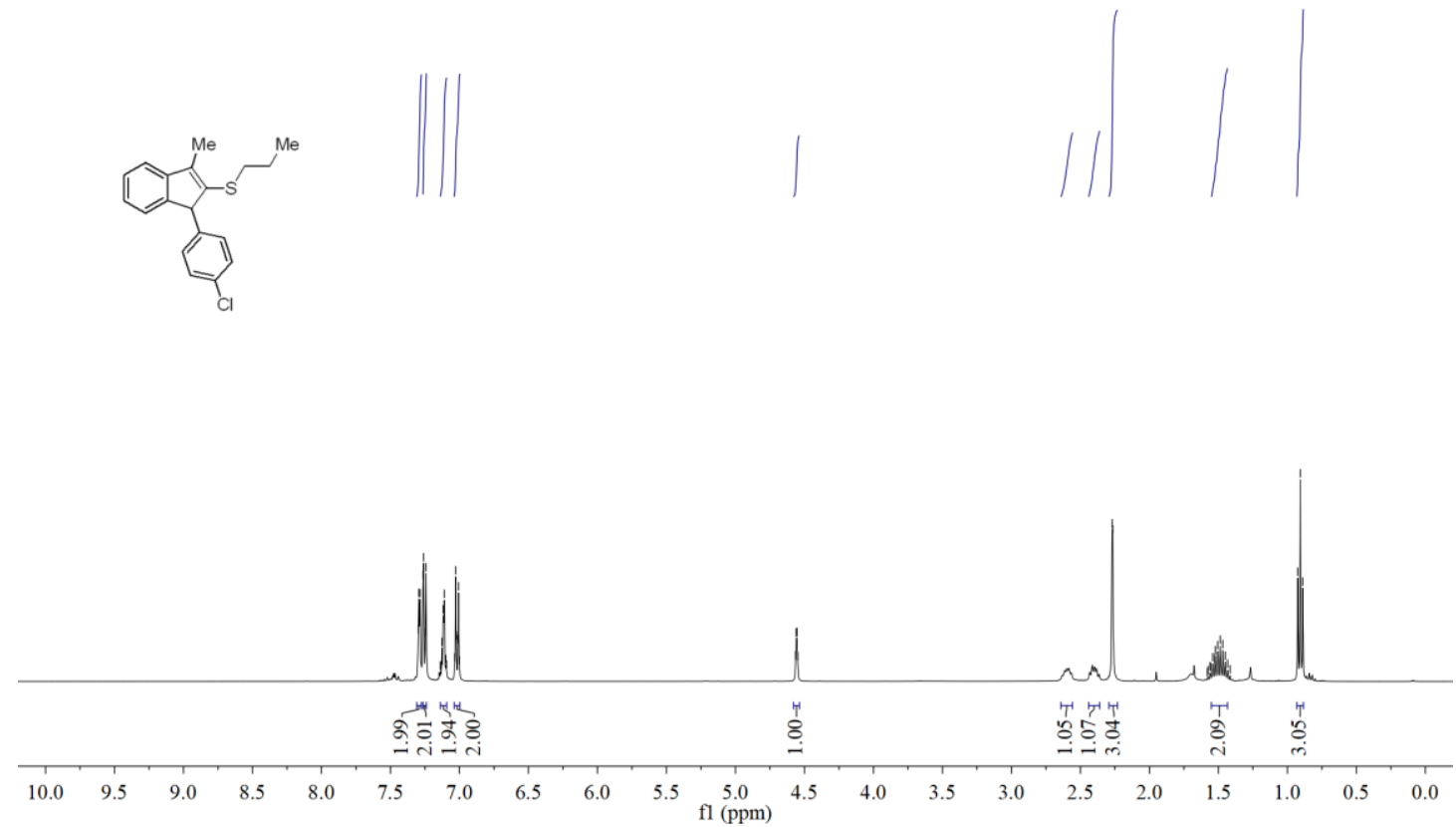

${ }^{13} \mathrm{C}$ NMR (101 MHz, $\left.\mathrm{CDCl}_{3}\right)$ spectrum of compound $\mathbf{3 m}$

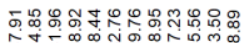

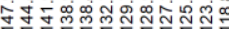

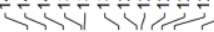

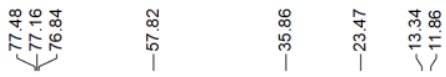
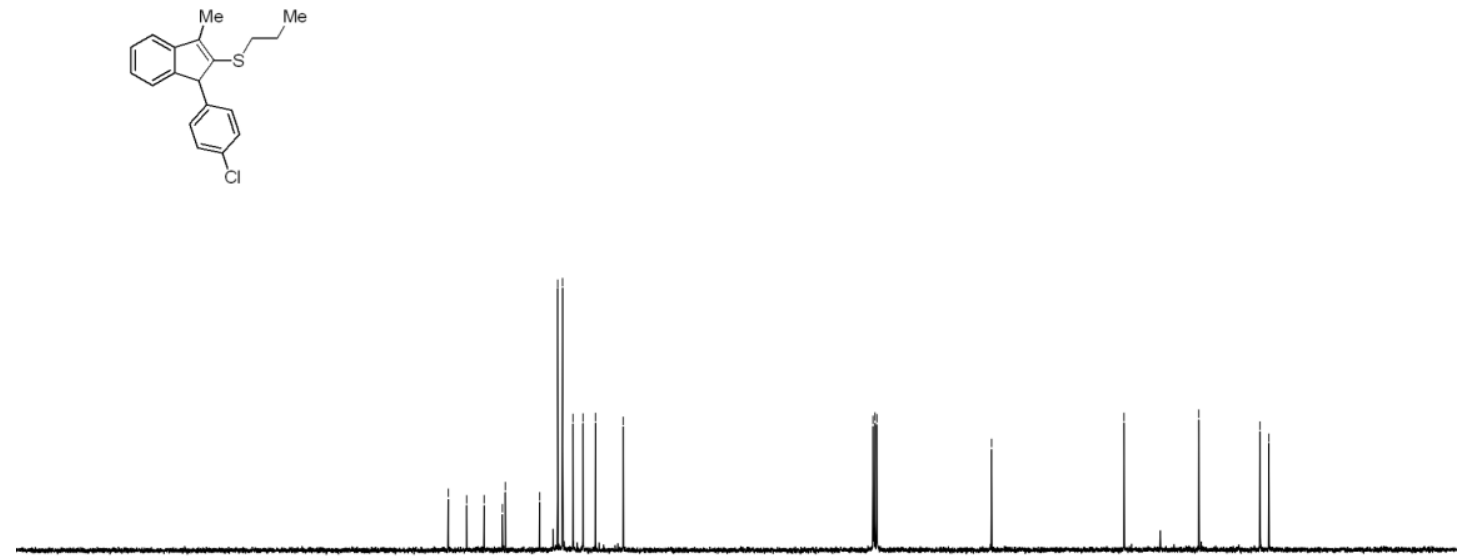
${ }^{1} \mathrm{H} \mathrm{NMR}\left(400 \mathrm{MHz}, \mathrm{CDCl}_{3}\right.$ ) spectrum of compound $\mathbf{3 n}$

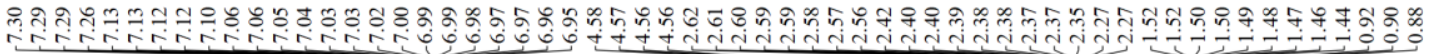

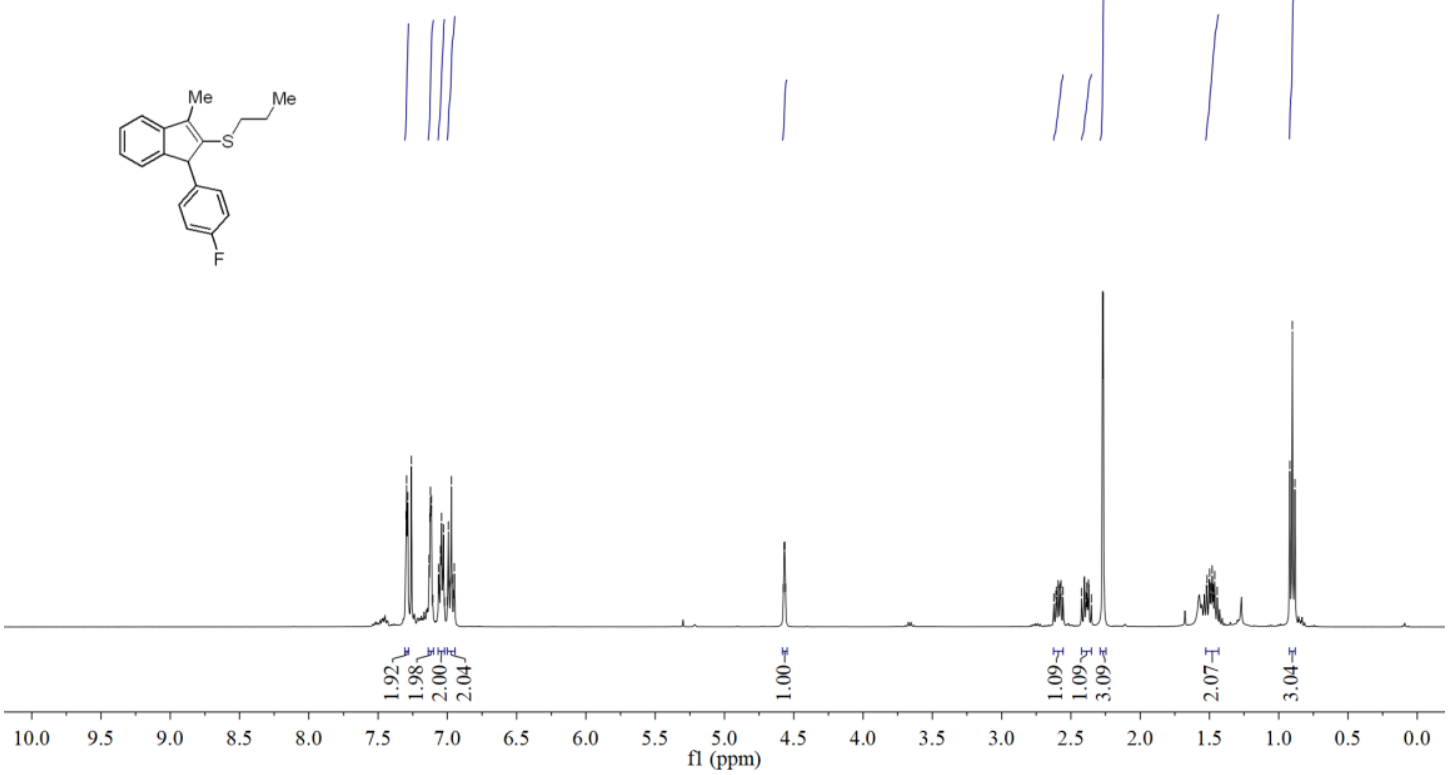

${ }^{13} \mathrm{C} \mathrm{NMR}\left(101 \mathrm{MHz}, \mathrm{CDCl}_{3}\right)$ spectrum of compound $\mathbf{3 n}$

유

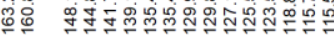

武每
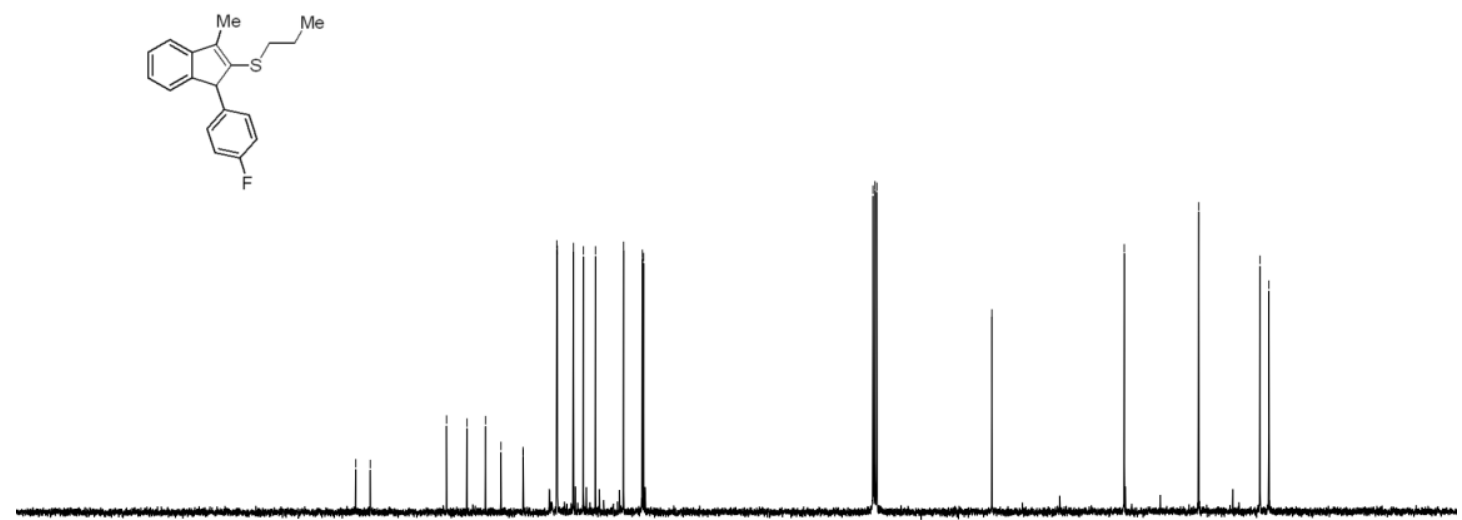
${ }^{1} \mathrm{H} \mathrm{NMR}\left(400 \mathrm{MHz}, \mathrm{CDCl}_{3}\right)$ spectrum of compound $\mathbf{3 0}$

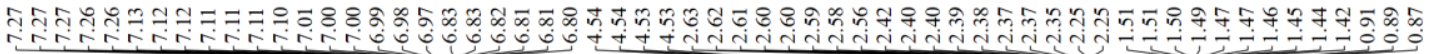

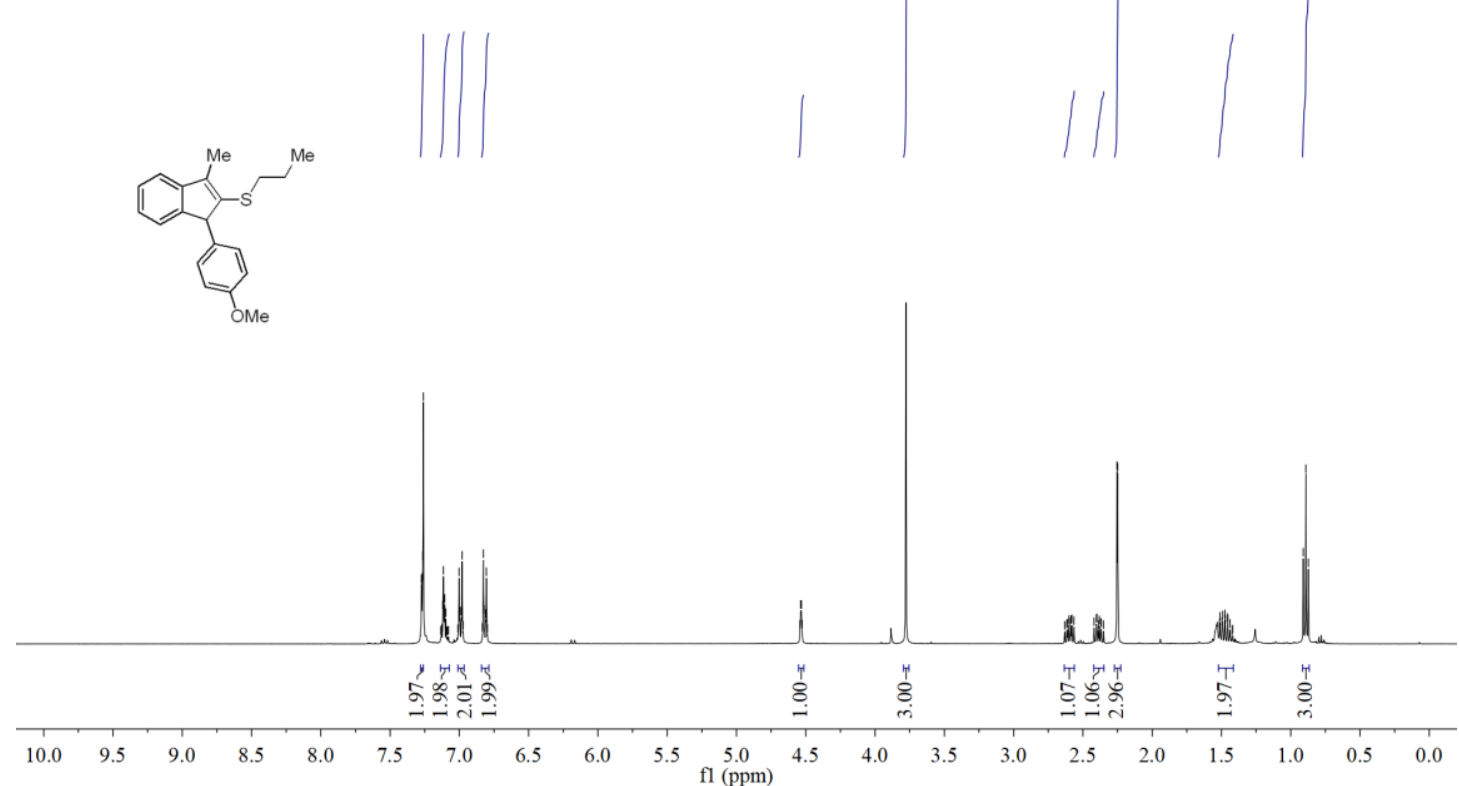

${ }^{13} \mathrm{C} \mathrm{NMR}\left(101 \mathrm{MHz}, \mathrm{CDCl}_{3}\right)$ spectrum of compound 30

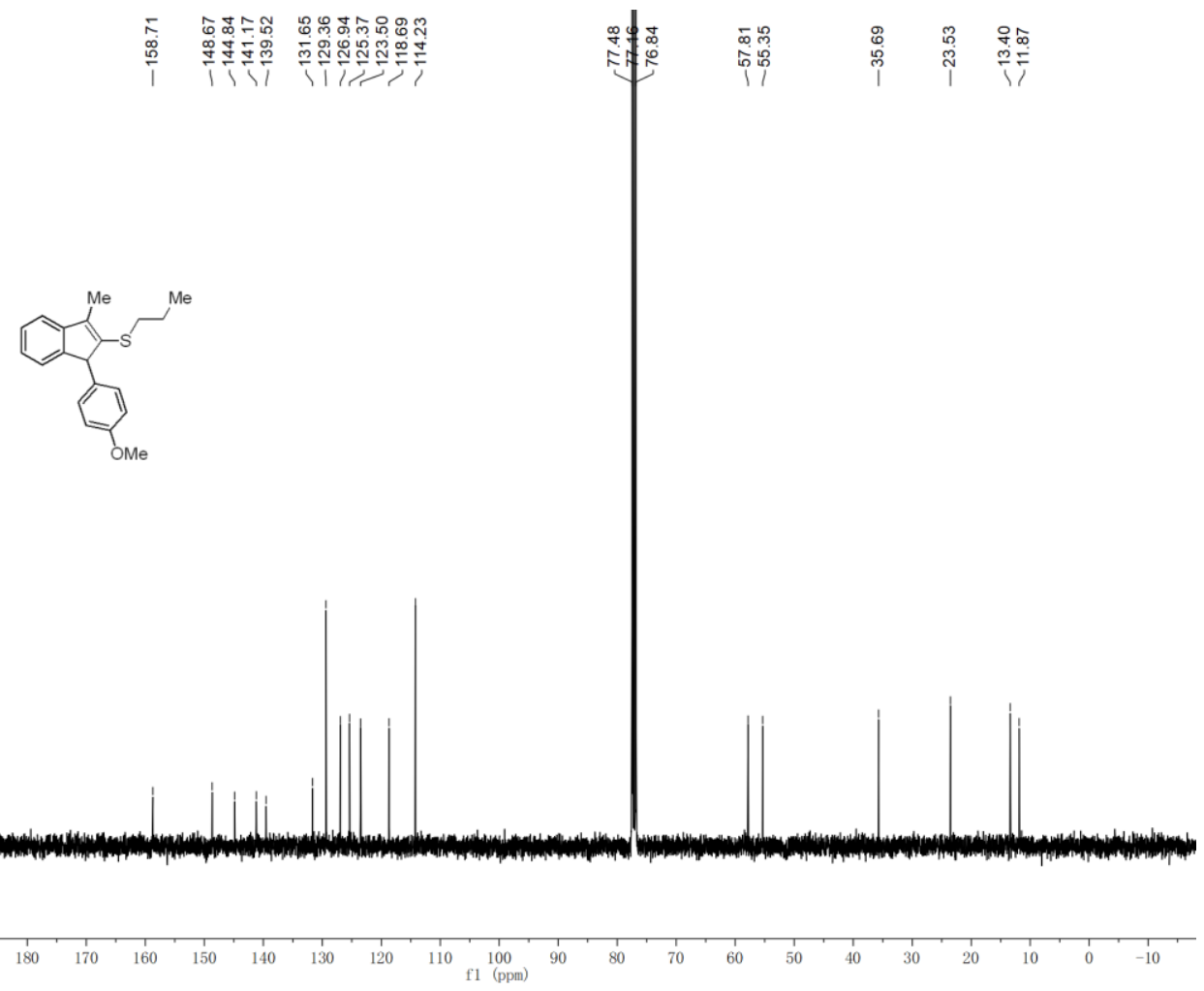


${ }^{1} \mathrm{H}$ NMR (400 MHz, $\mathrm{CDCl}_{3}$ ) spectrum of compound $\mathbf{3 p}$

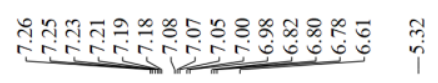

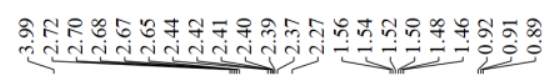

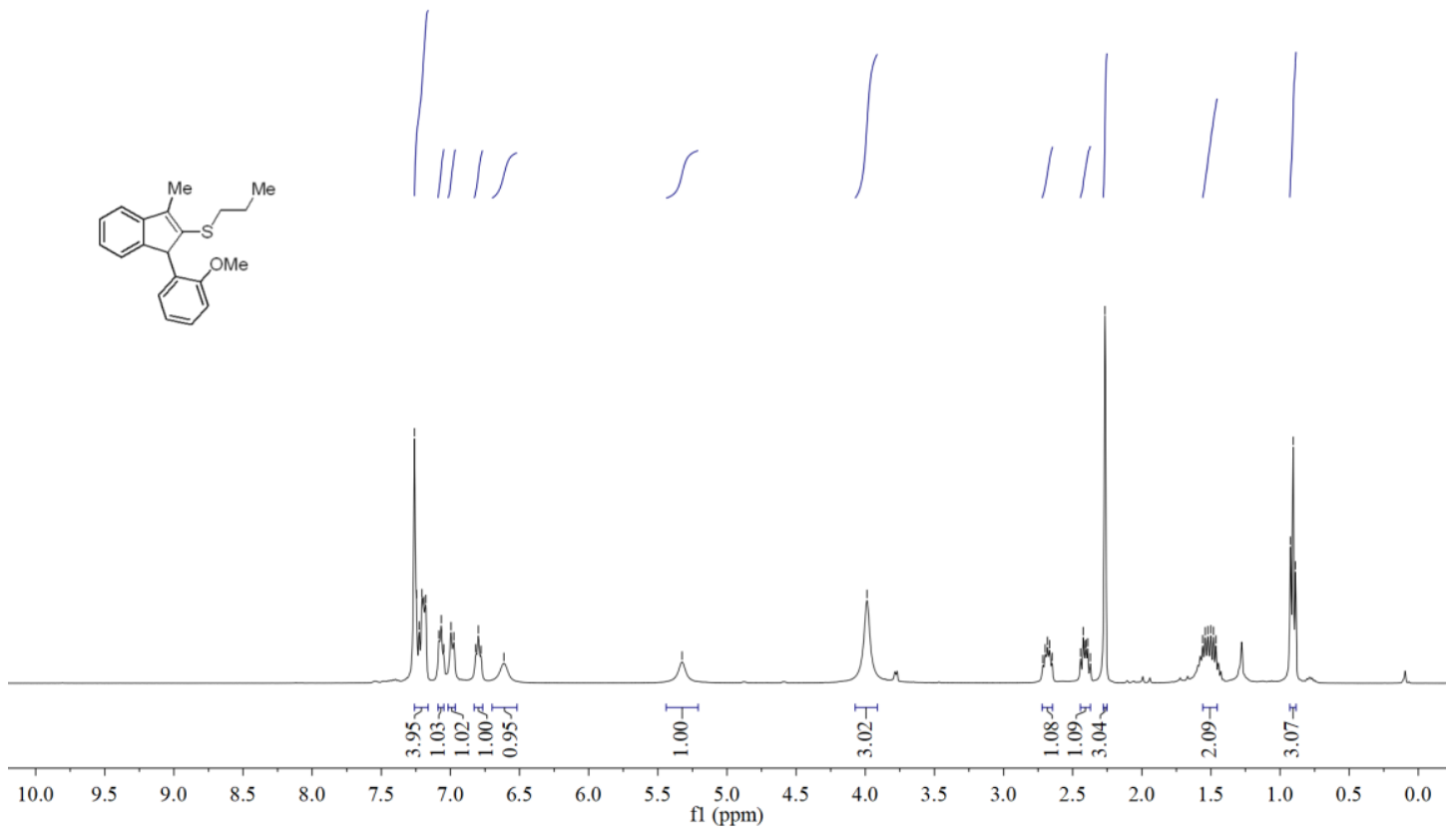

${ }^{13} \mathrm{C} \mathrm{NMR}\left(101 \mathrm{MHz}, \mathrm{CDCl}_{3}\right)$ spectrum of compound $\mathbf{3 p}$

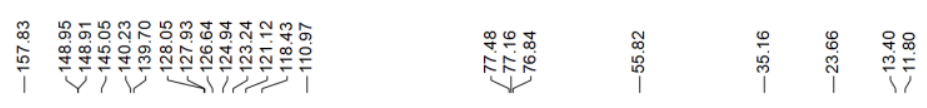
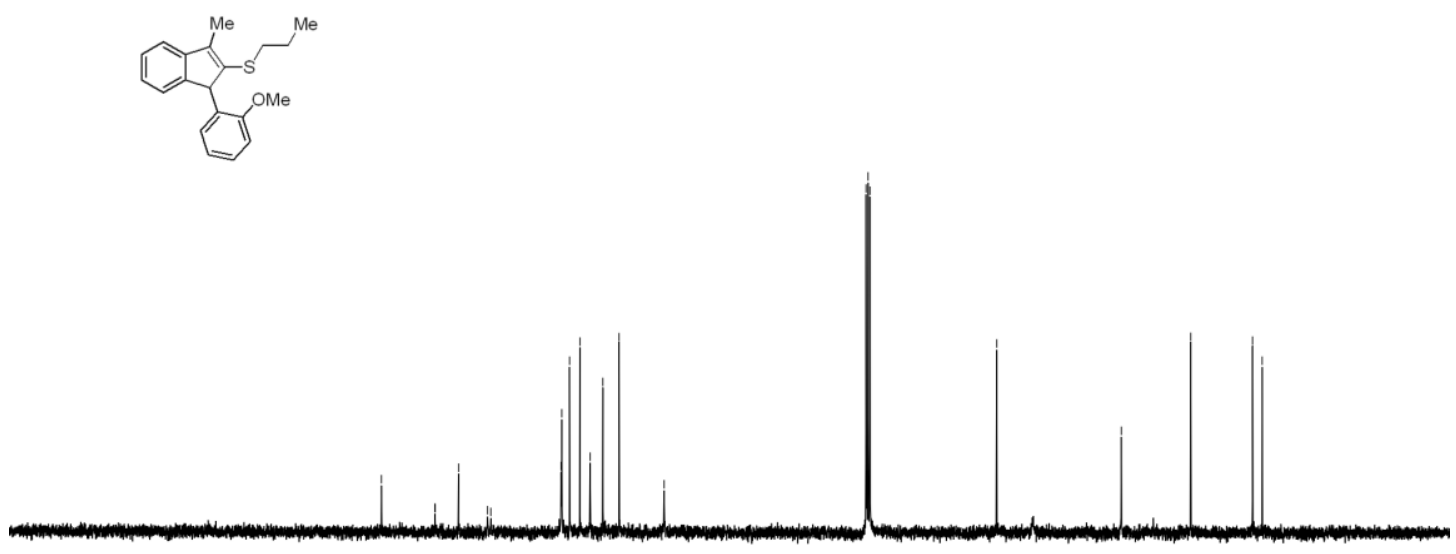

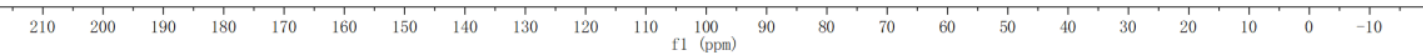

S53 
${ }^{1} \mathrm{H}$ NMR (400 MHz, $\mathrm{CDCl}_{3}$ ) spectrum of compound $\mathbf{3 q}$

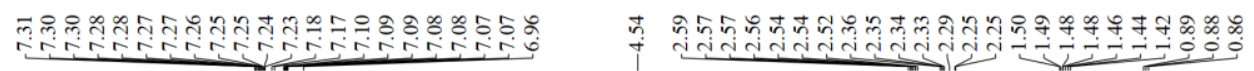
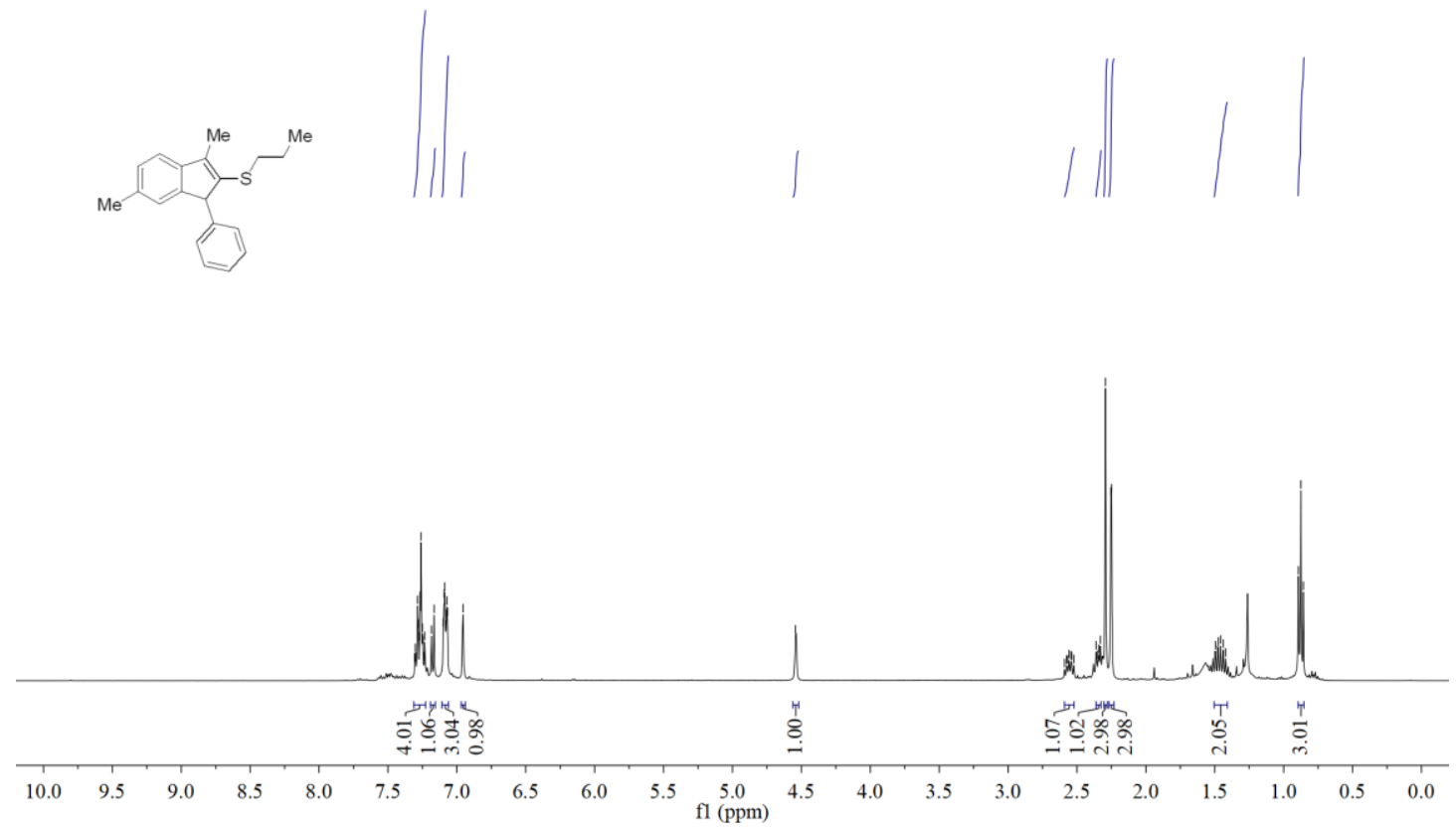

${ }^{13} \mathrm{C} \mathrm{NMR}\left(101 \mathrm{MHz}, \mathrm{CDCl}_{3}\right)$ spectrum of compound $\mathbf{3 q}$

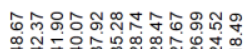

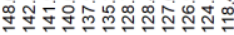

TiT

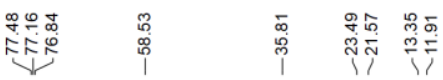

(Me

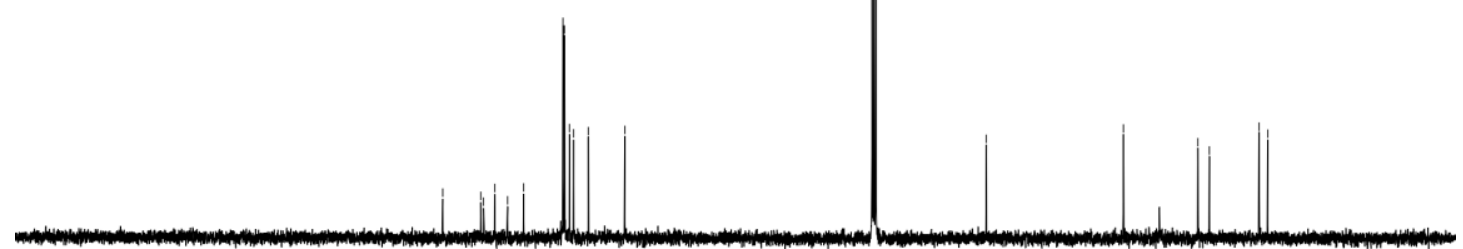

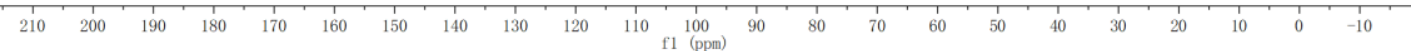


${ }^{1} \mathrm{H}$ NMR (400 MHz, $\left.\mathrm{CDCl}_{3}\right)$ spectrum of compound $\mathbf{3 r}$

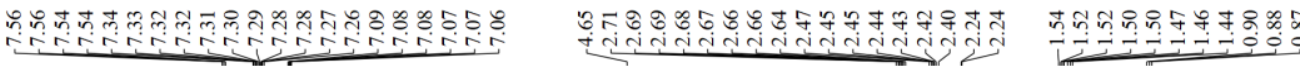
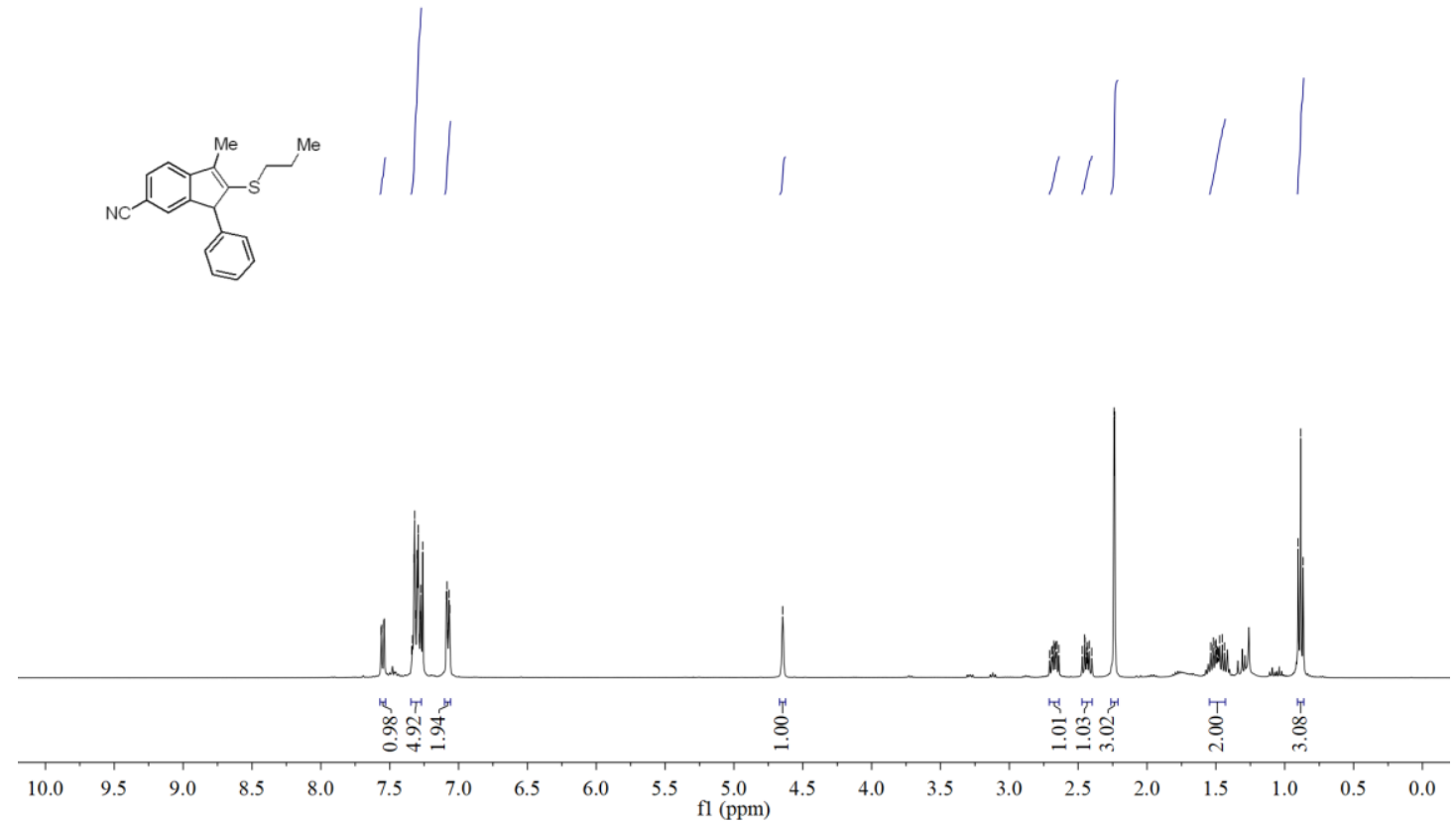

${ }^{13} \mathrm{C} \mathrm{NMR}\left(101 \mathrm{MHz}, \mathrm{CDCl}_{3}\right)$ spectrum of compound $\mathbf{3 r}$

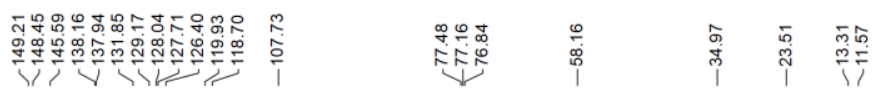
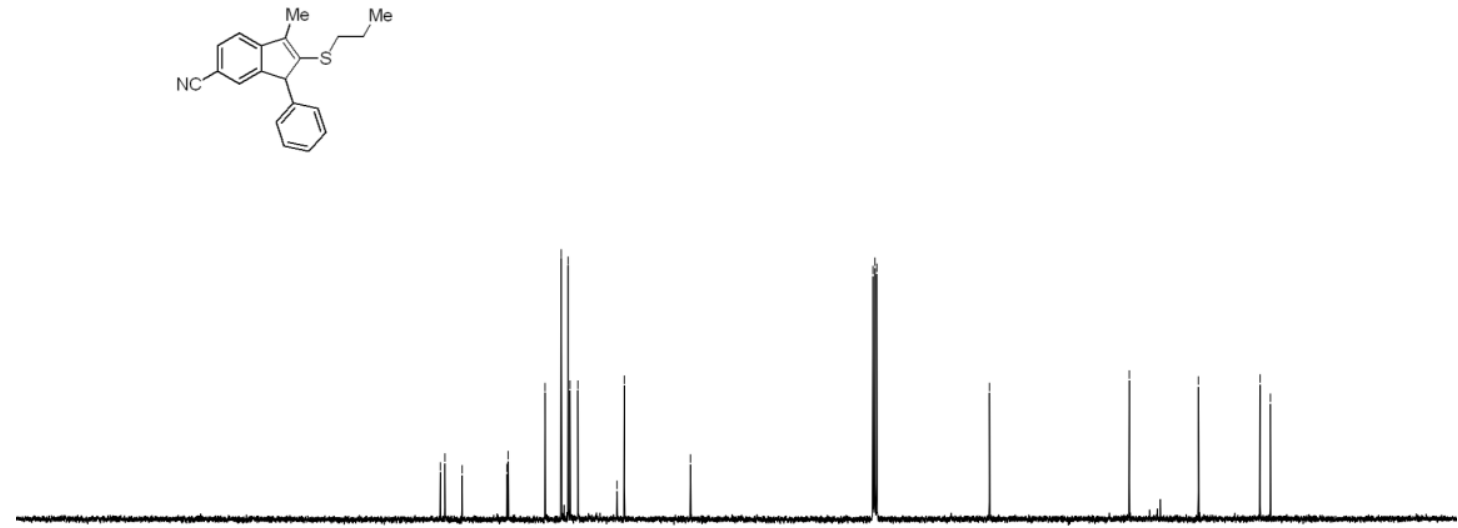
${ }^{1} \mathrm{H} \mathrm{NMR}\left(400 \mathrm{MHz}, \mathrm{CDCl}_{3}\right)$ spectrum of compound 3s

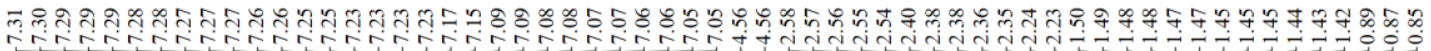
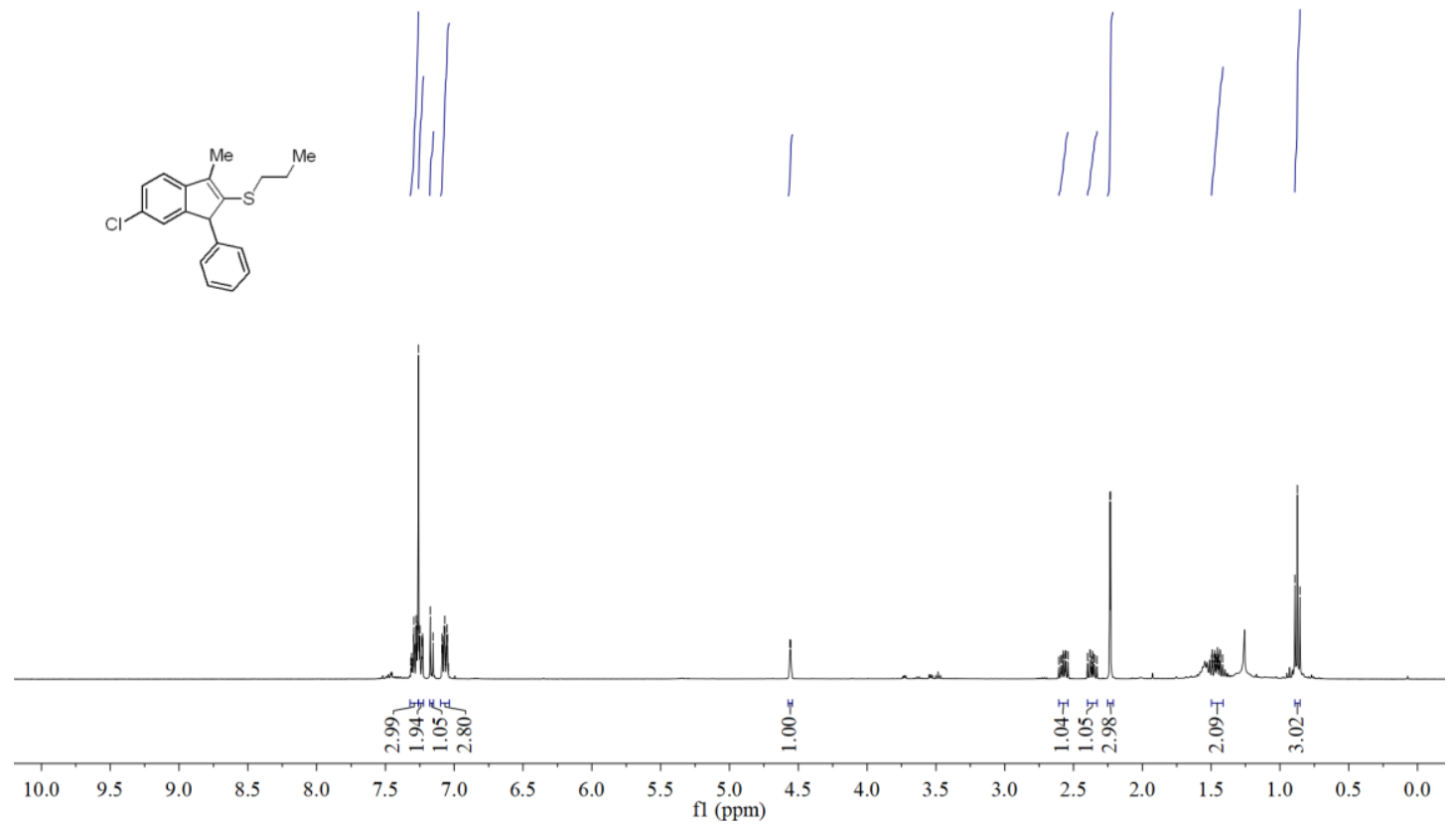

${ }^{13} \mathrm{C} \mathrm{NMR}\left(101 \mathrm{MHz}, \mathrm{CDCl}_{3}\right)$ spectrum of compound $\mathbf{3 s}$

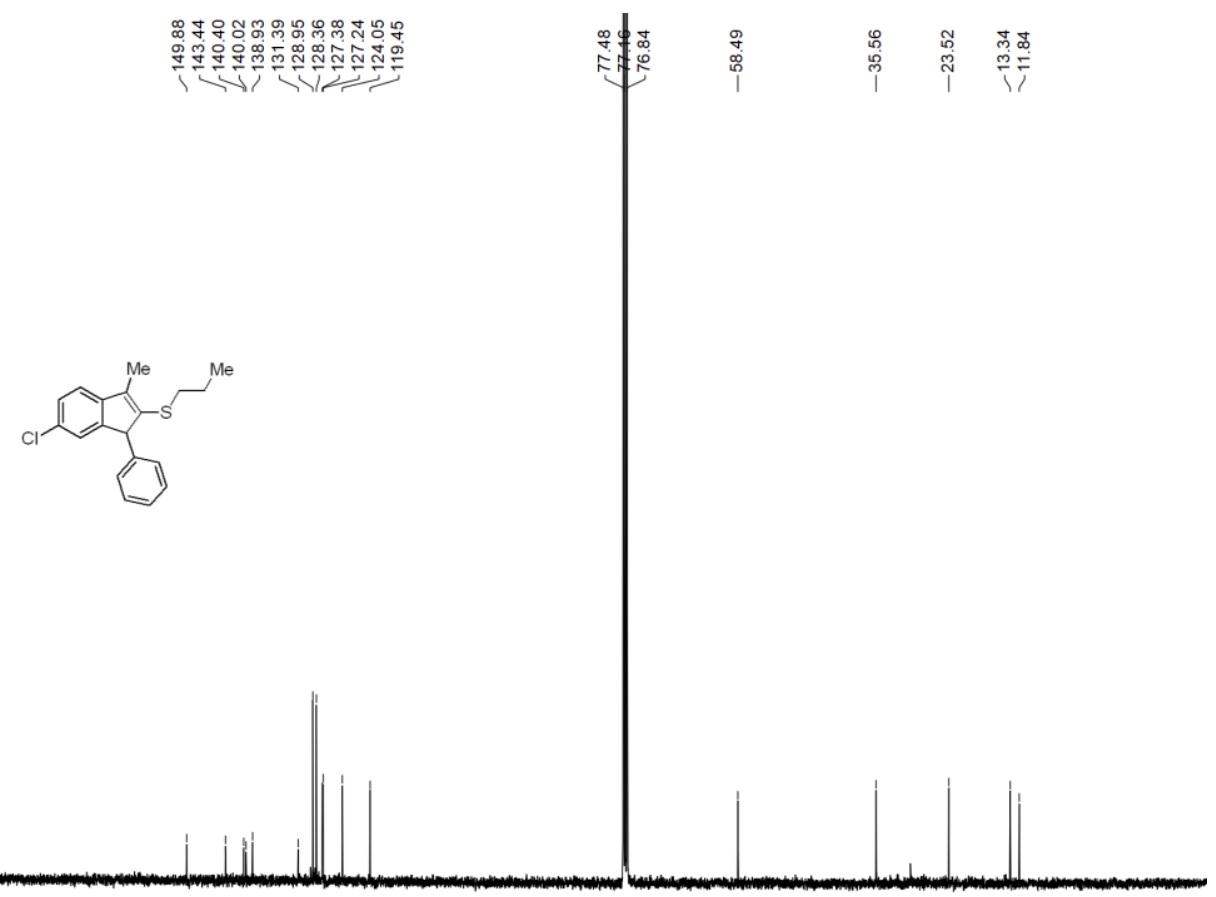

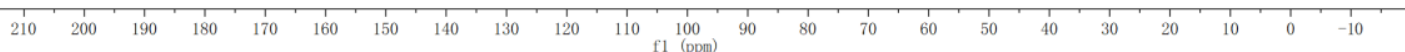


${ }^{1} \mathrm{H} \mathrm{NMR}\left(400 \mathrm{MHz}, \mathrm{CDCl}_{3}\right)$ spectrum of compound $\mathbf{3 t}$

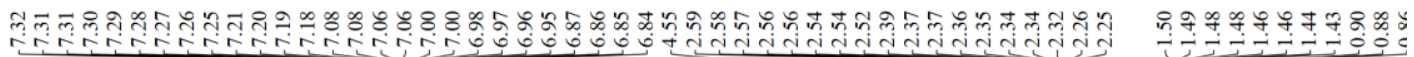
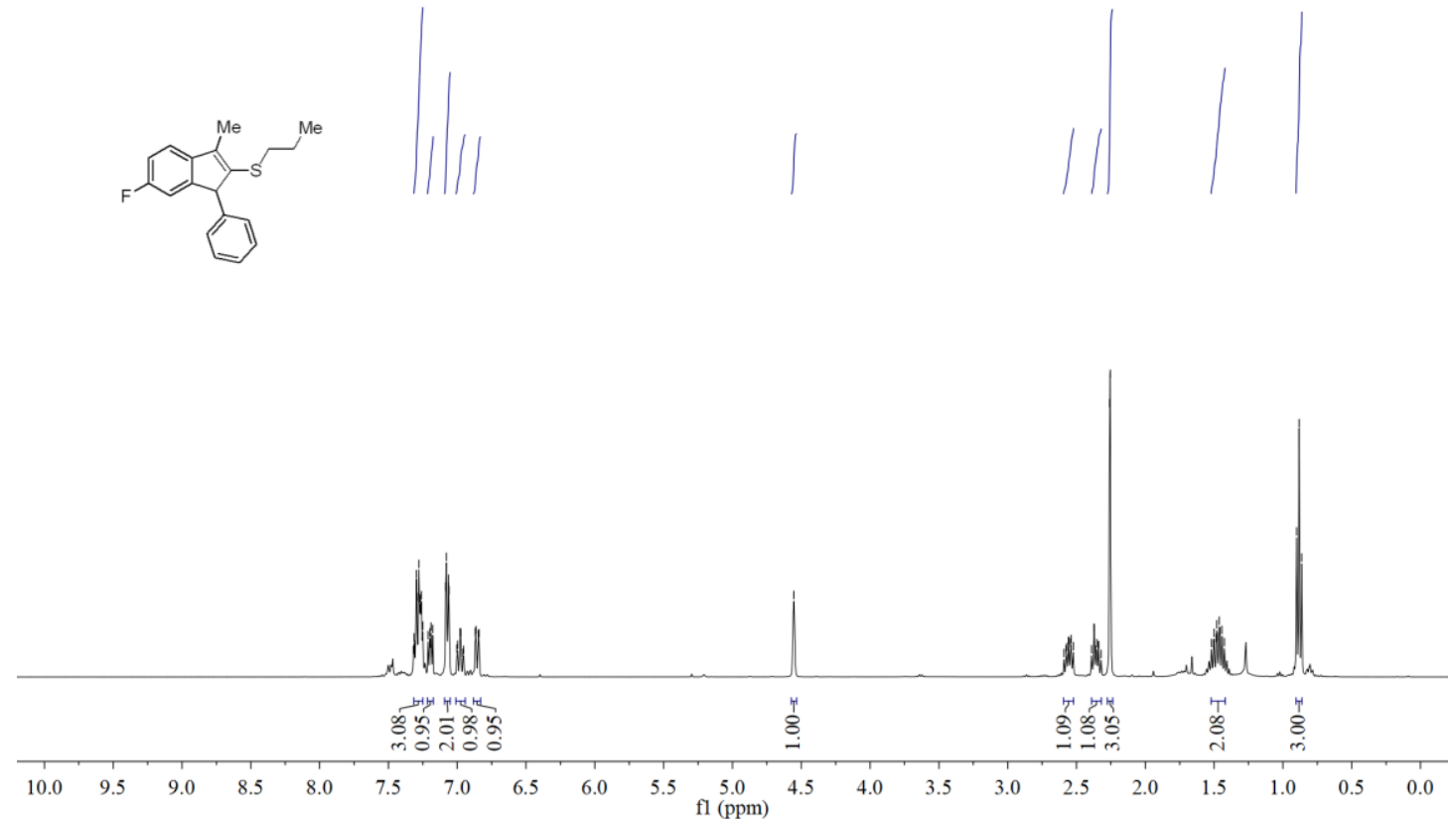

${ }^{13} \mathrm{C} \mathrm{NMR}\left(101 \mathrm{MHz}, \mathrm{CDCl}_{3}\right)$ spectrum of compound $\mathbf{3 t}$

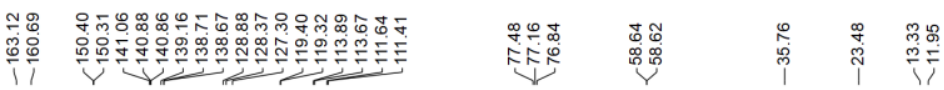

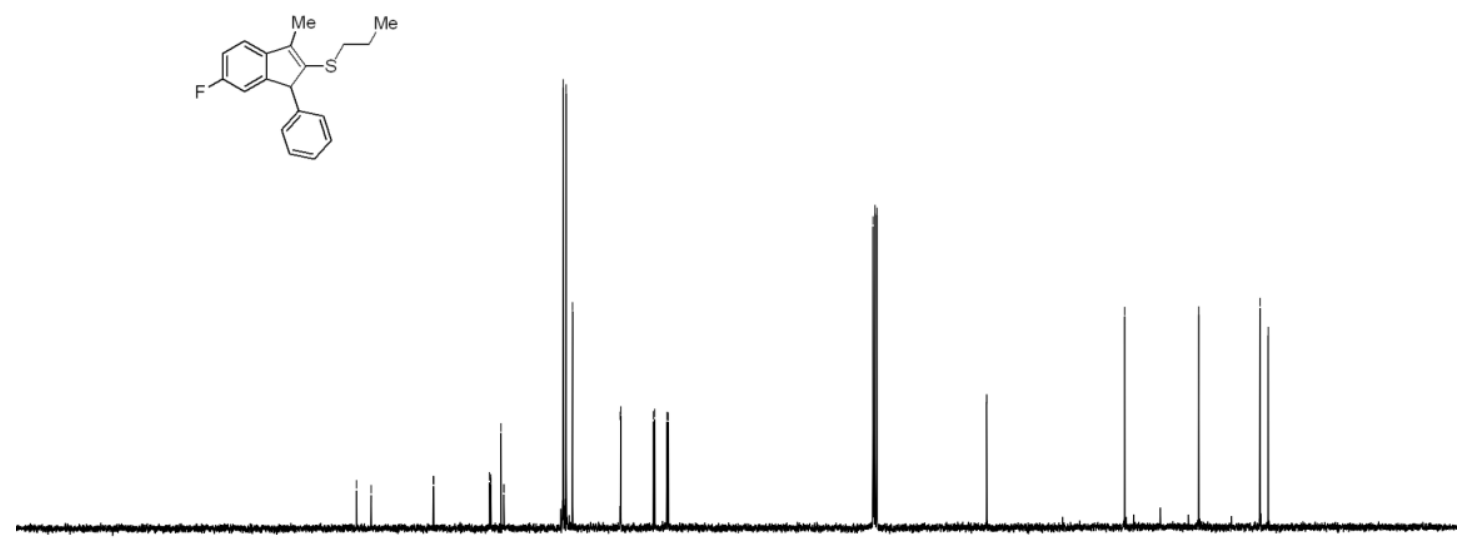

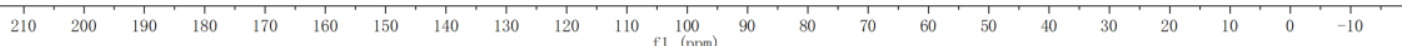


${ }^{1} \mathrm{H} \mathrm{NMR}\left(400 \mathrm{MHz}, \mathrm{CDCl}_{3}\right)$ spectrum of compound $\mathbf{3 u}$

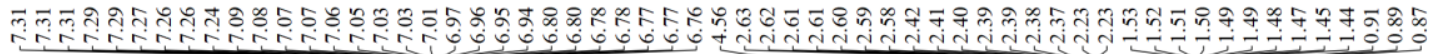
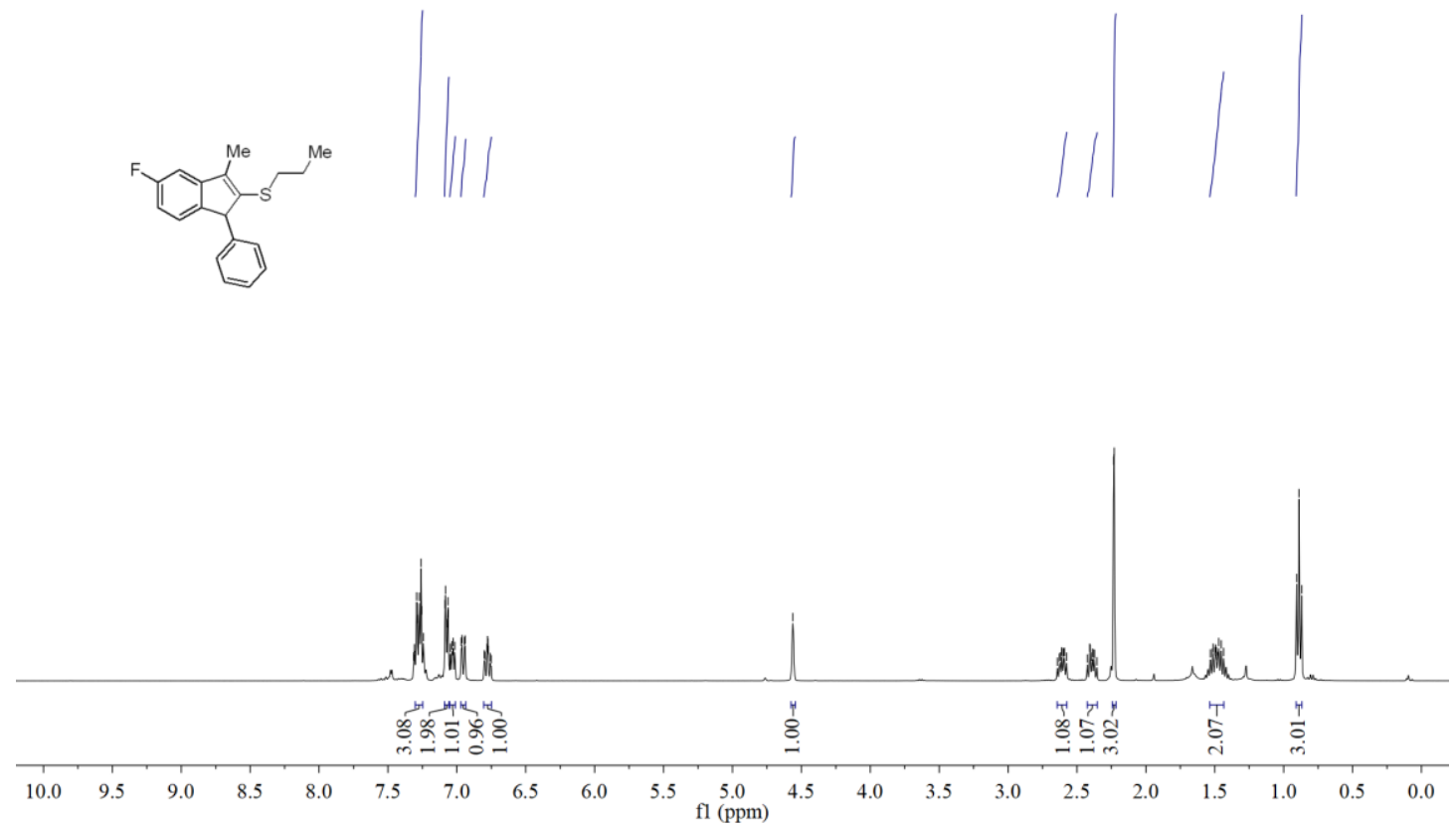

${ }^{13} \mathrm{C} \mathrm{NMR}\left(101 \mathrm{MHz}, \mathrm{CDCl}_{3}\right)$ spectrum of compound $\mathbf{3 u}$

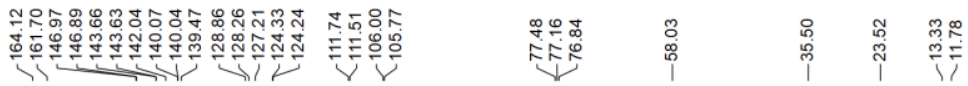
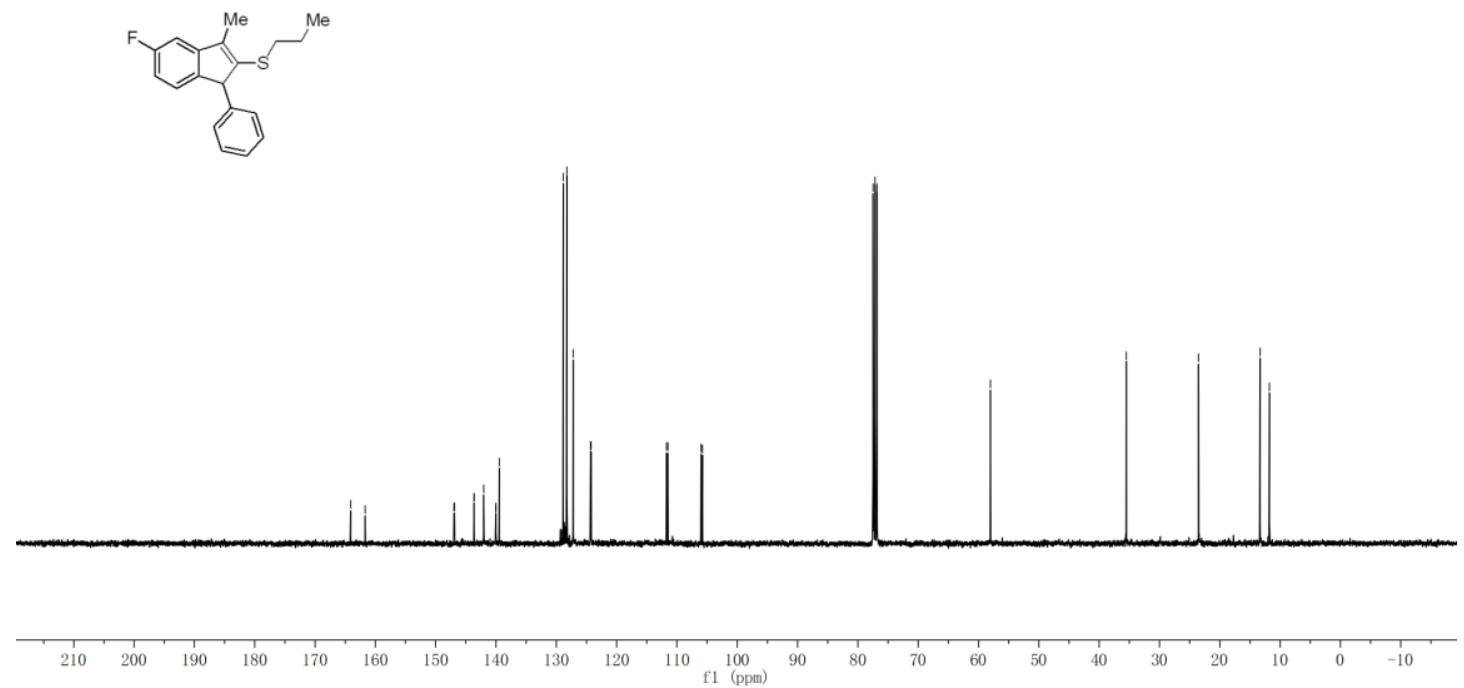
${ }^{1} \mathrm{H}$ NMR (400 MHz, $\left.\mathrm{CDCl}_{3}\right)$ spectrum of compound $\mathbf{3 v}$

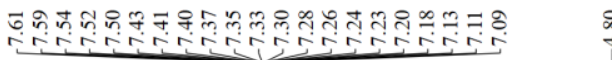

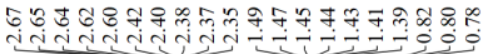
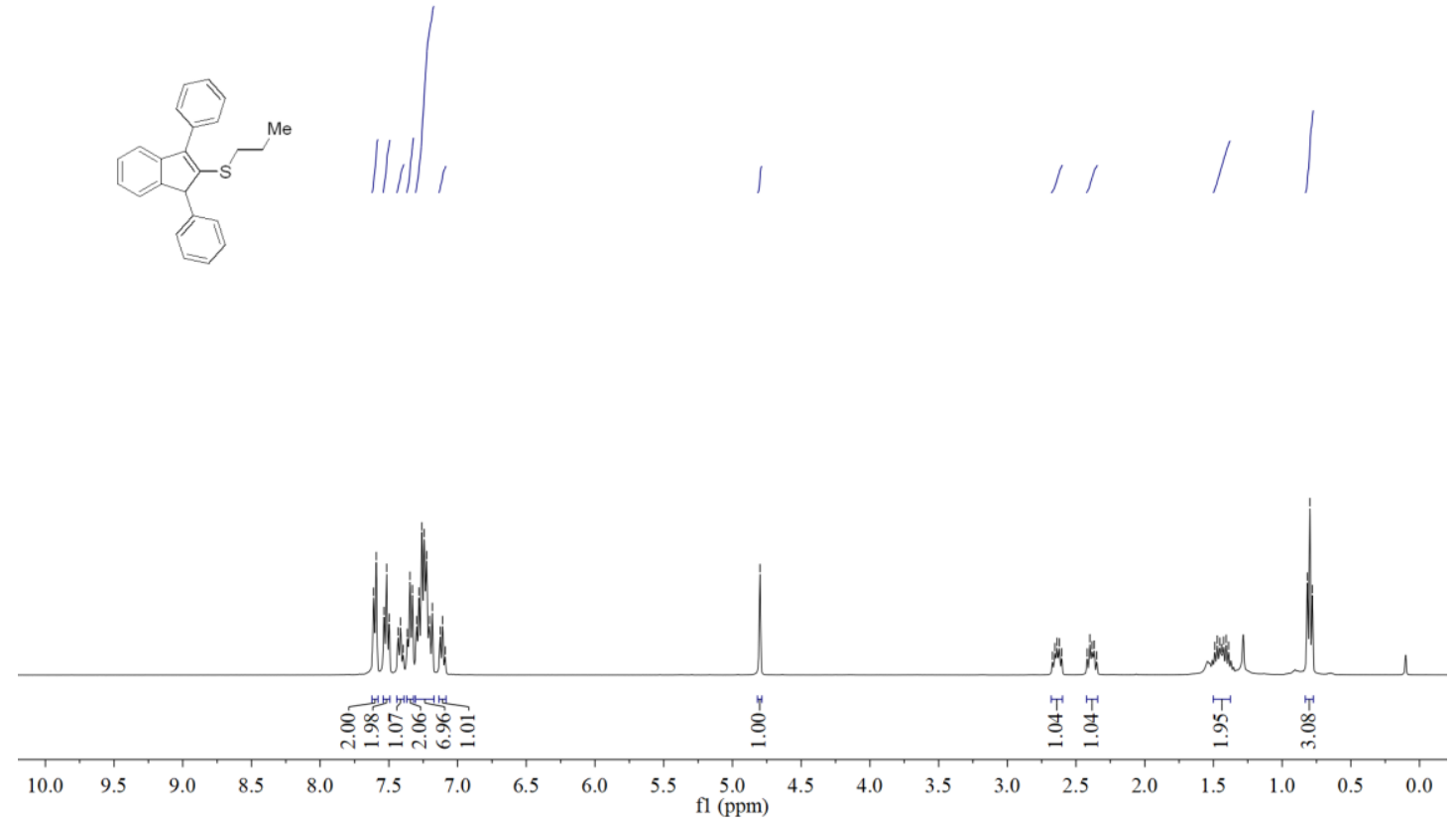

${ }^{13} \mathrm{C} \mathrm{NMR}\left(101 \mathrm{MHz}, \mathrm{CDCl}_{3}\right)$ spectrum of compound $\mathbf{3 v}$

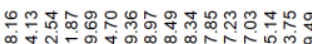

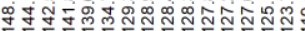
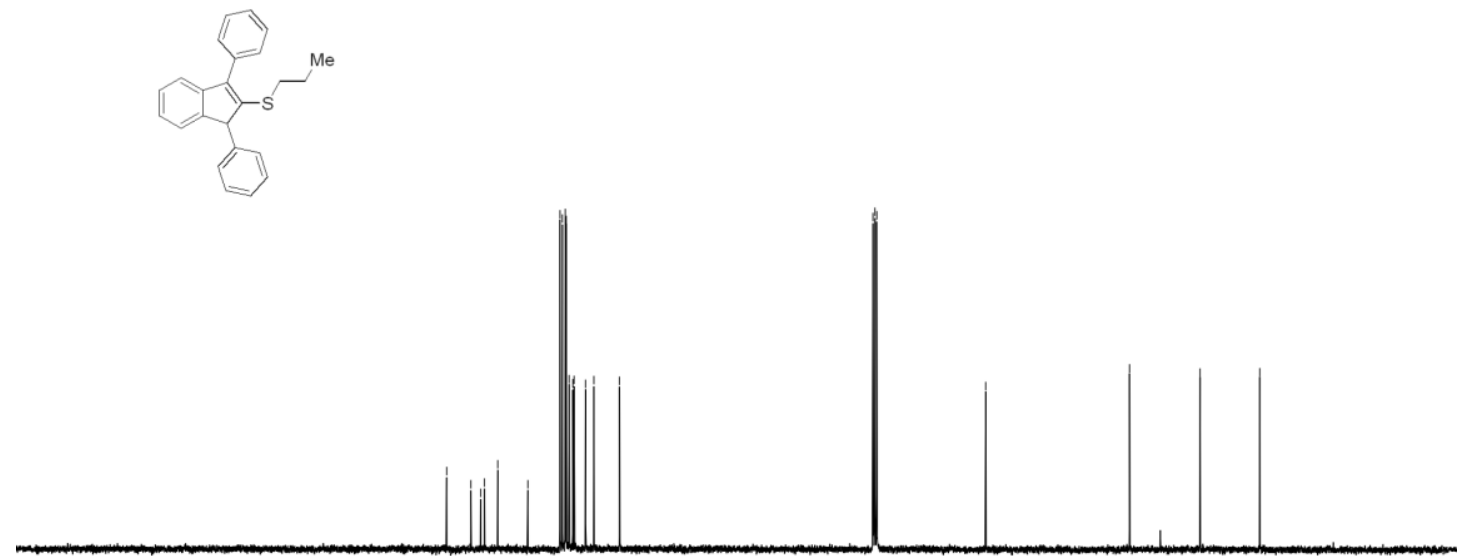
${ }^{1} \mathrm{H}$ NMR (400 MHz, $\left.\mathrm{CDCl}_{3}\right)$ spectrum of compound $\mathbf{3 w}$

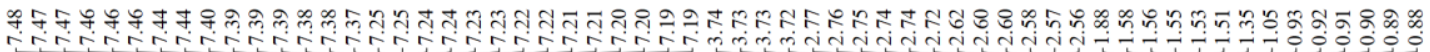
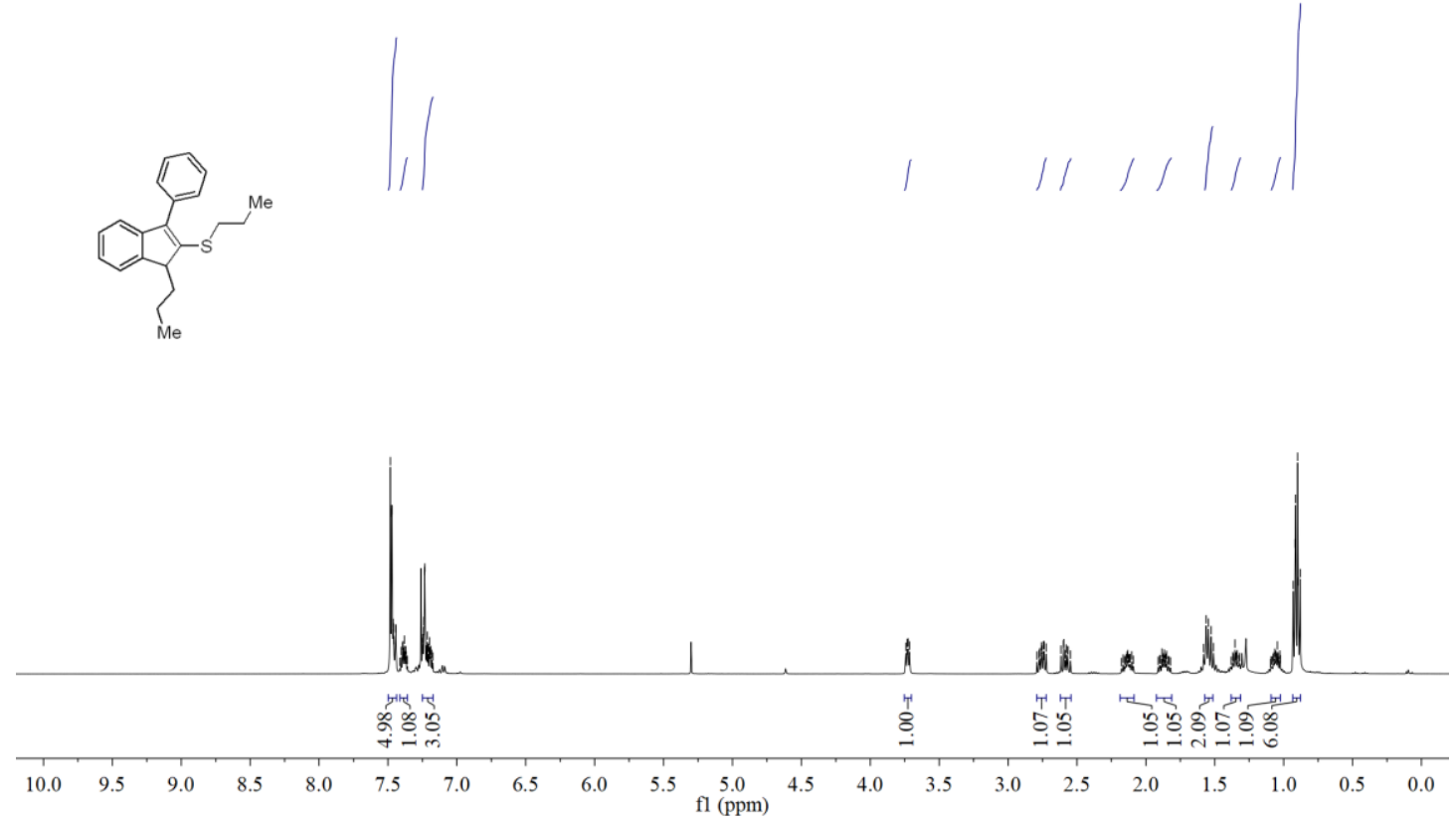

${ }^{13} \mathrm{C} \mathrm{NMR}\left(101 \mathrm{MHz}, \mathrm{CDCl}_{3}\right)$ spectrum of compound $\mathbf{3 w}$

\begin{tabular}{|c|c|c|c|}
\hline 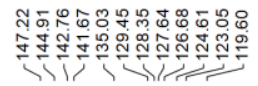 & 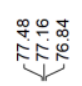 & $\frac{1}{5}$ & 尺िए \\
\hline
\end{tabular}
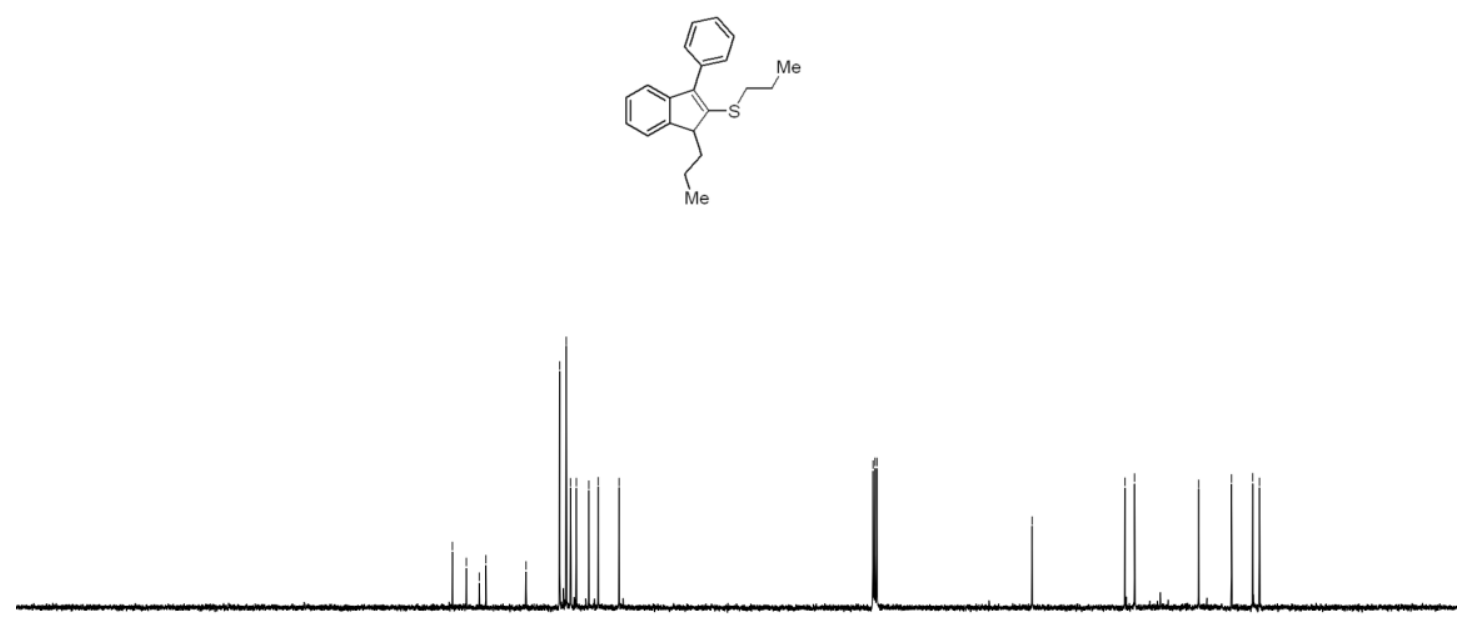

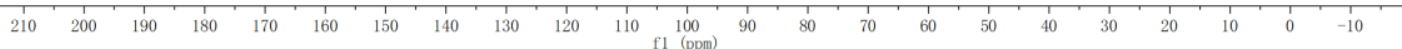


${ }^{1} \mathrm{H}$ NMR (400 MHz, $\left.\mathrm{CDCl}_{3}\right)$ spectrum of compound $\mathbf{3 x}$

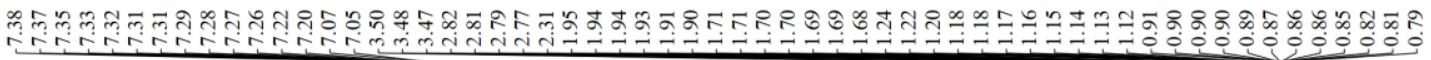

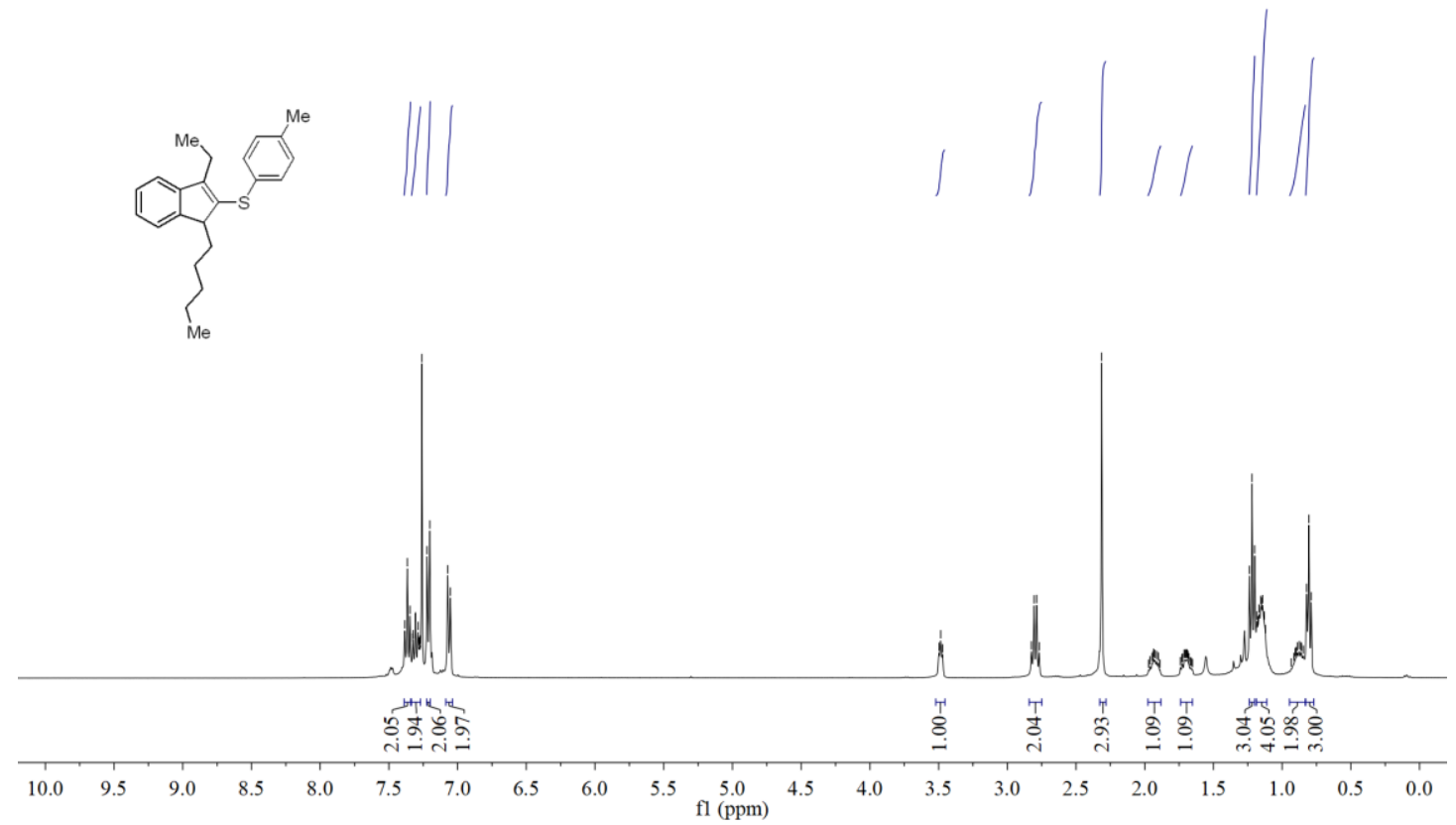

${ }^{13} \mathrm{C} \mathrm{NMR}\left(101 \mathrm{MHz}, \mathrm{CDCl}_{3}\right)$ spectrum of compound $\mathbf{3 x}$

\begin{tabular}{|c|c|}
\hline 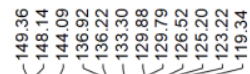 & 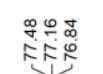 \\
\hline
\end{tabular}
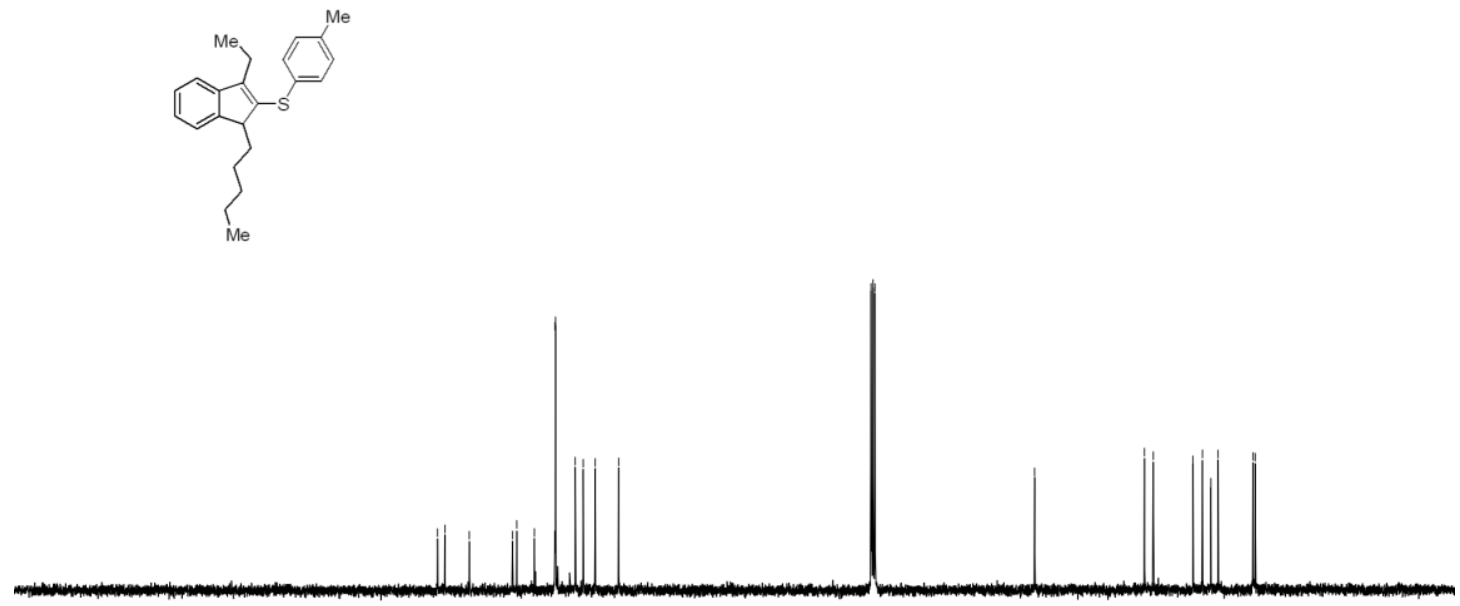

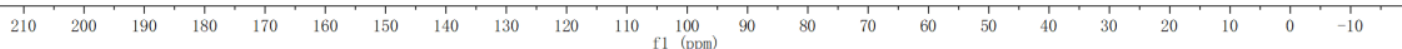


${ }^{1} \mathrm{H}$ NMR (400 MHz, $\mathrm{CDCl}_{3}$ ) spectrum of compound $\mathbf{3 y}$

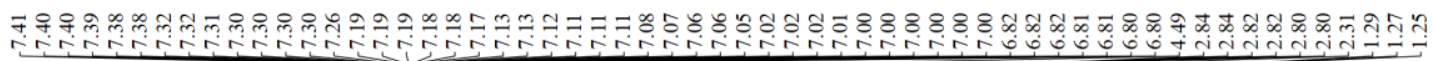

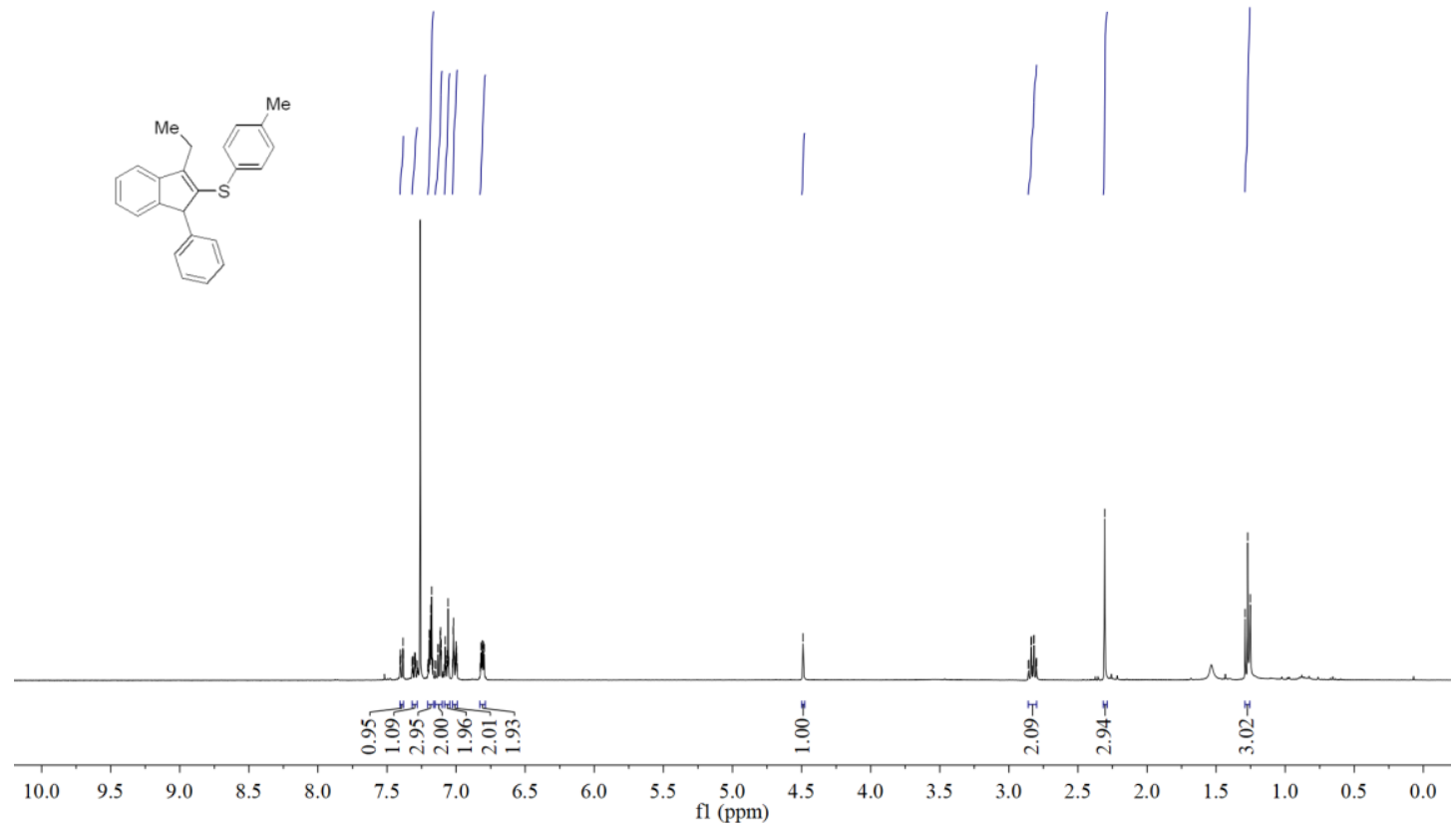

${ }^{13} \mathrm{C} \mathrm{NMR}\left(101 \mathrm{MHz}, \mathrm{CDCl}_{3}\right)$ spectrum of compound $\mathbf{3 y}$

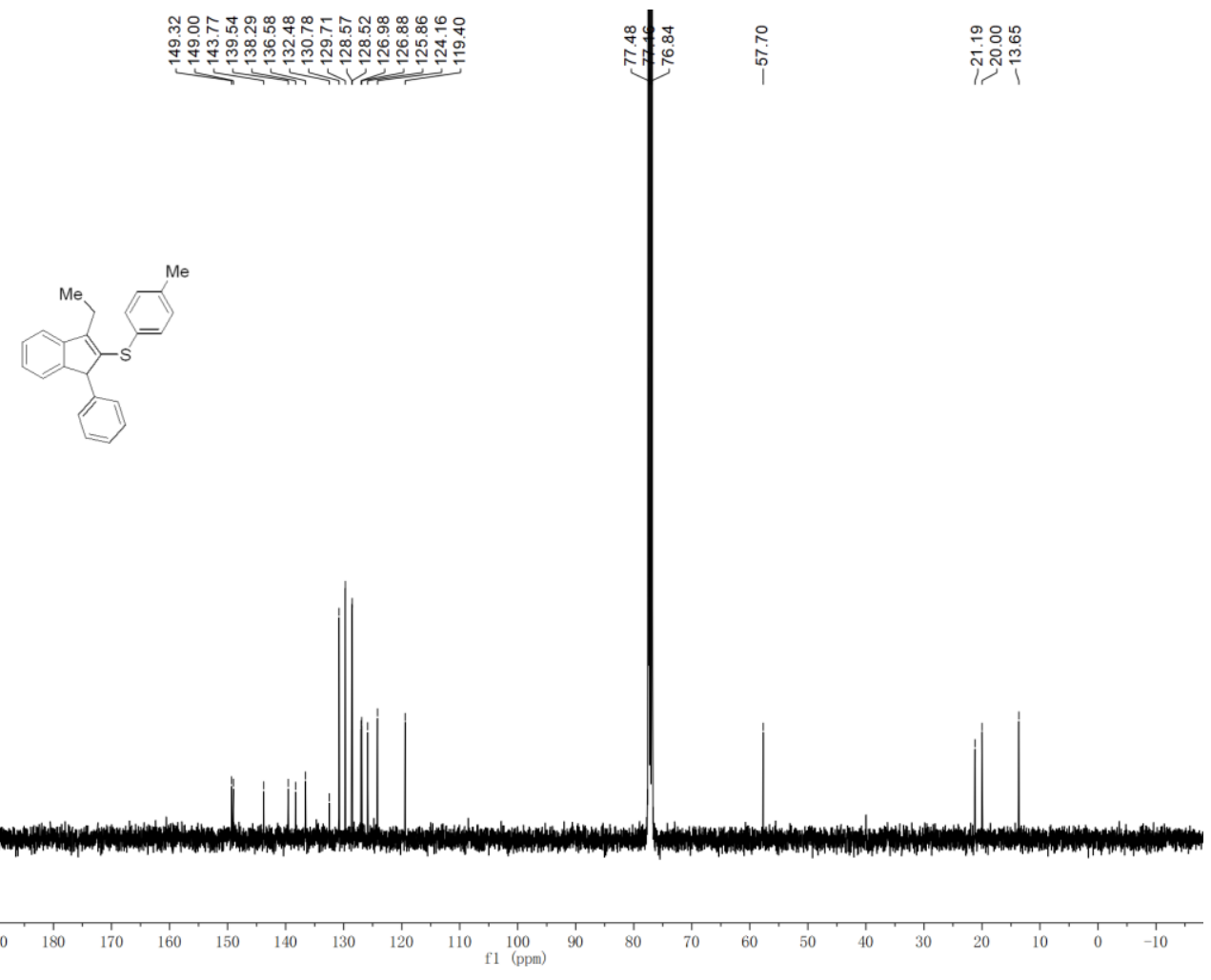


${ }^{1} \mathrm{H}$ NMR (400 MHz, $\mathrm{CDCl}_{3}$ ) spectrum of compound $\mathbf{3 z}$

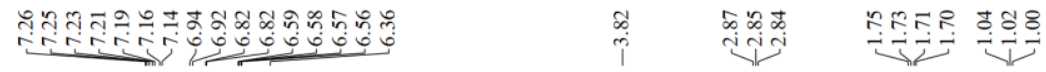

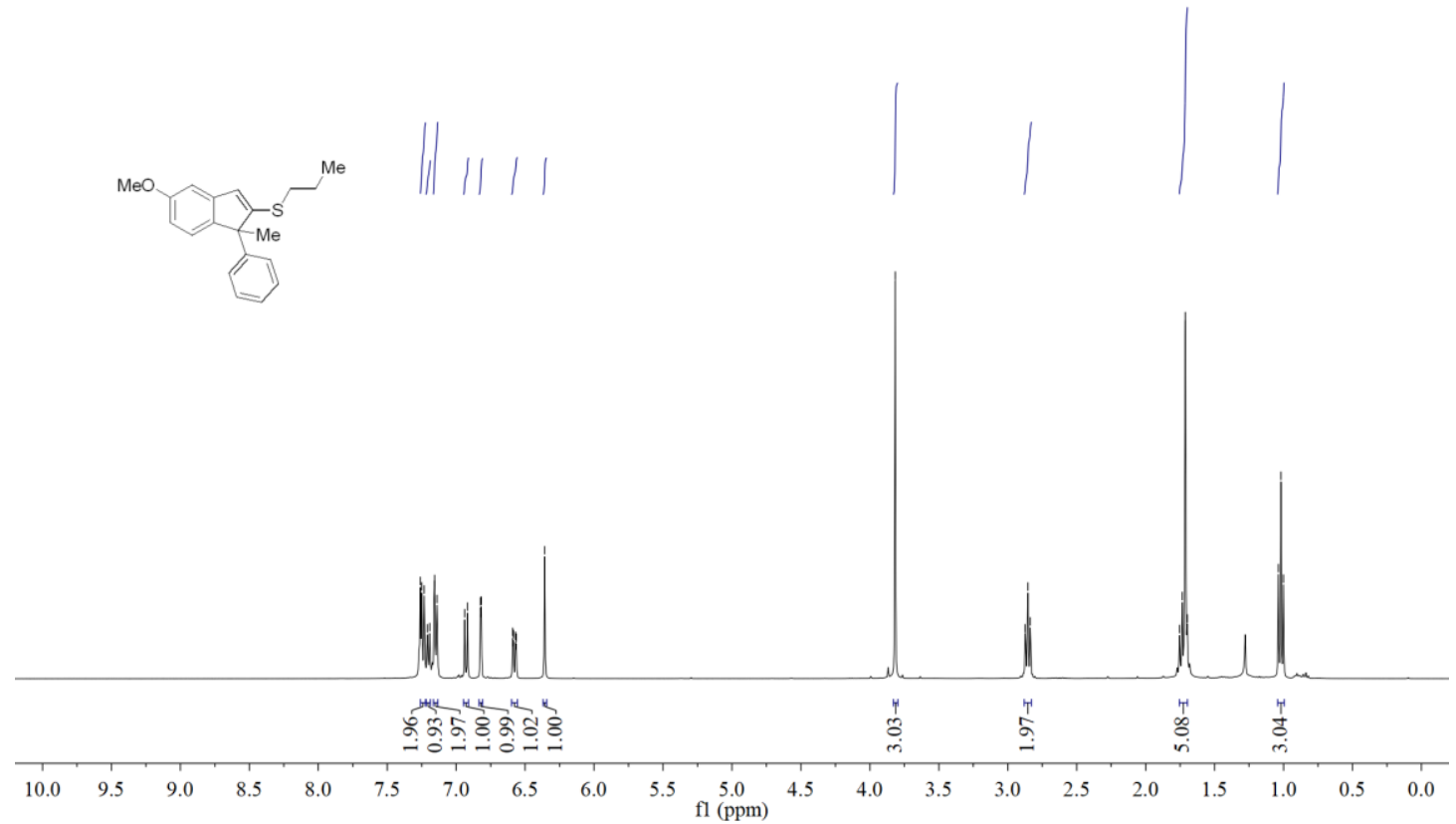

${ }^{13} \mathrm{C}$ NMR (101 MHz, $\left.\mathrm{CDCl}_{3}\right)$ spectrum of compound $\mathbf{3 z}$

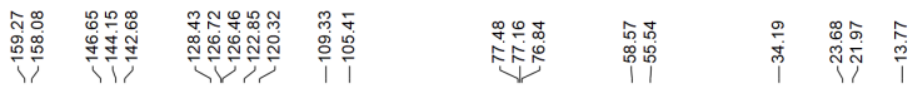
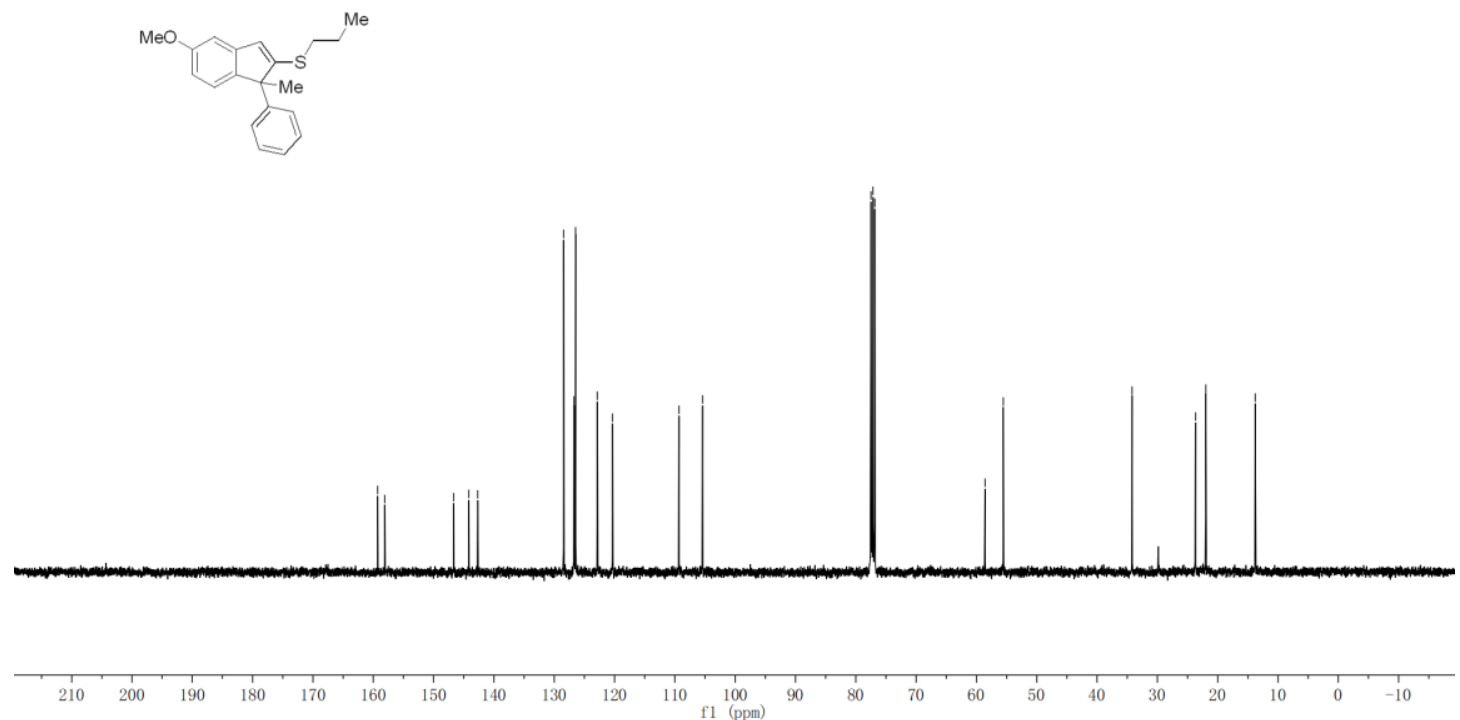
${ }^{1} \mathrm{H}$ NMR $\left(400 \mathrm{MHz}, \mathrm{CDCl}_{3}\right)$ spectrum of compound 3aa

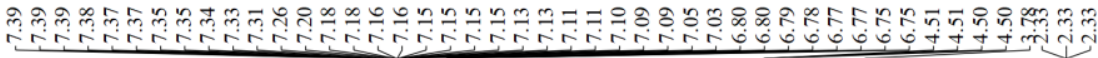

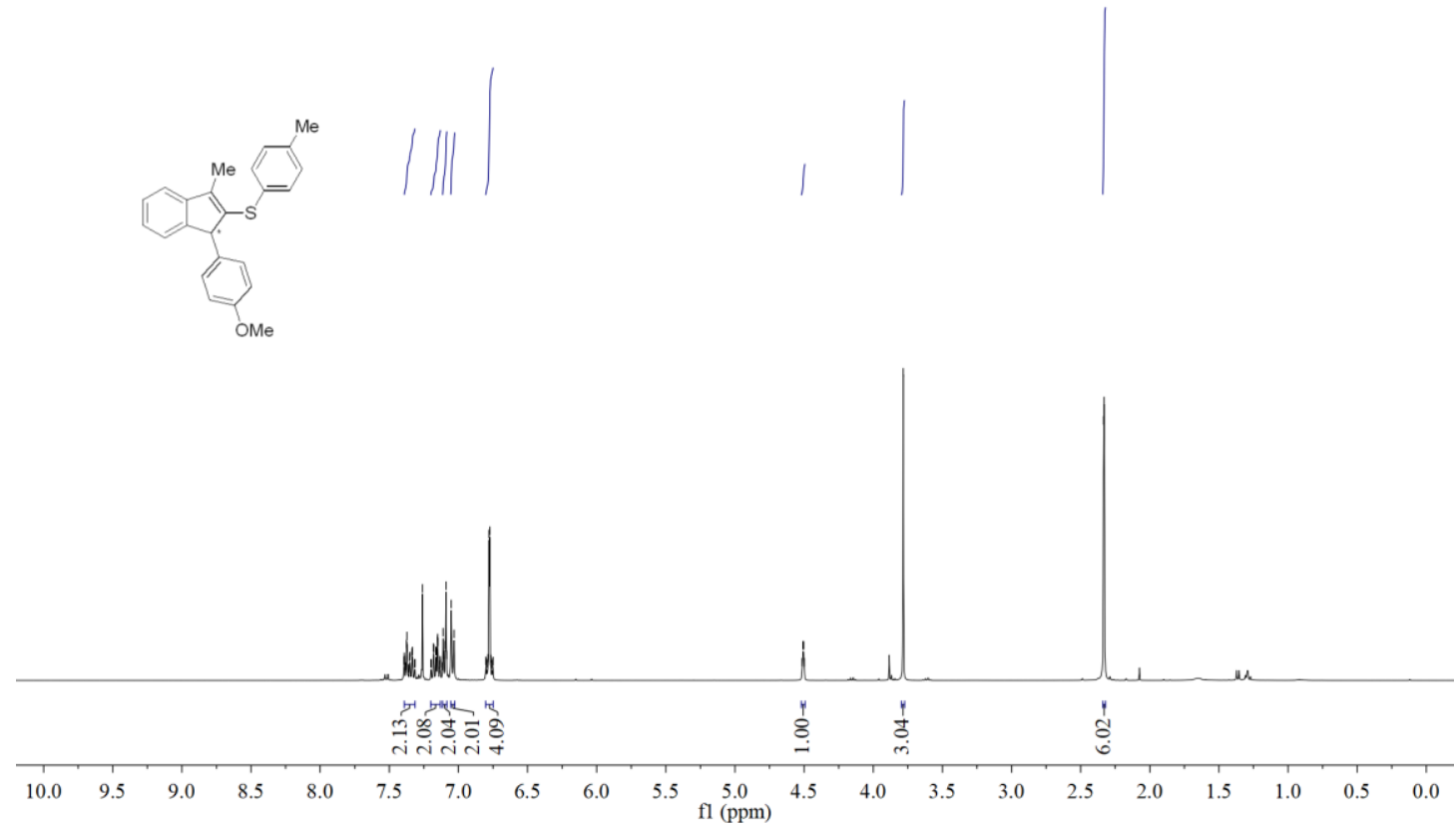

${ }^{13} \mathrm{C}$ NMR (101 MHz, $\left.\mathrm{CDCl}_{3}\right)$ spectrum of compound 3aa

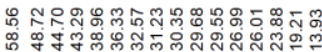

位

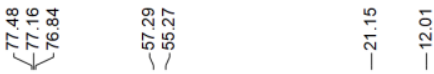
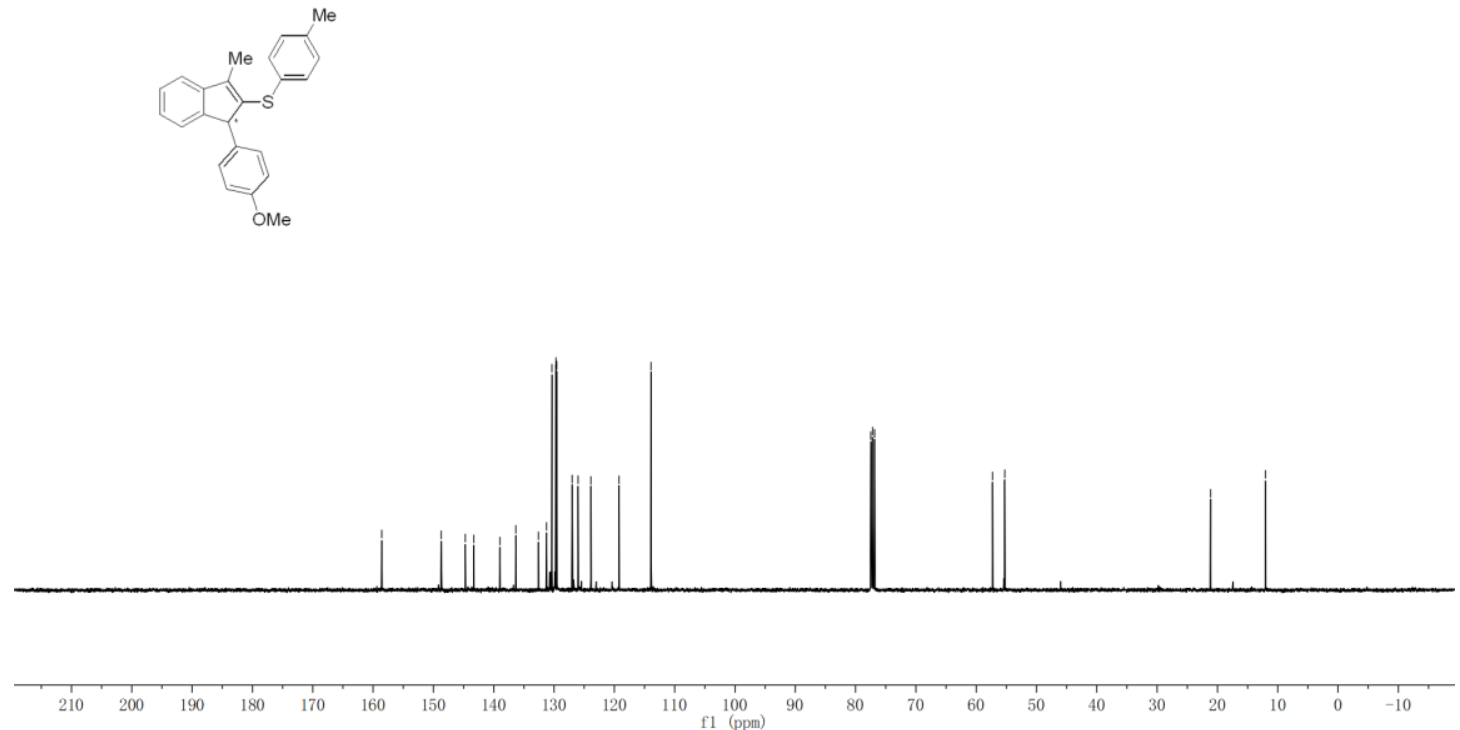
${ }^{1} \mathrm{H} \mathrm{NMR}\left(400 \mathrm{MHz}, \mathrm{CDCl}_{3}\right)$ spectrum of compound 4

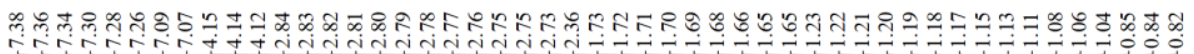
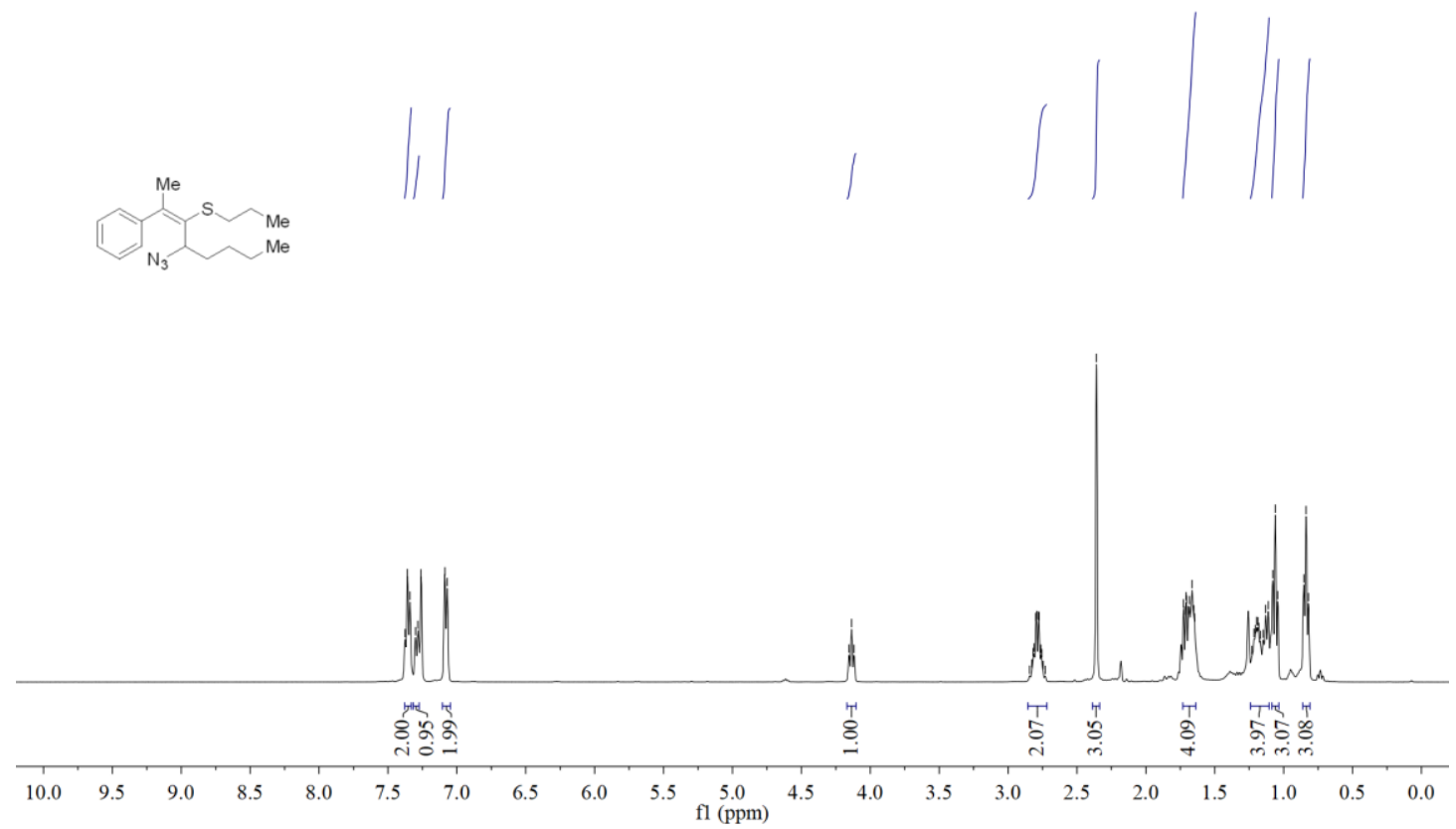

${ }^{13} \mathrm{C} \mathrm{NMR}\left(101 \mathrm{MHz}, \mathrm{CDCl}_{3}\right)$ spectrum of compound 4

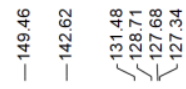

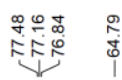

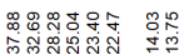

mijng ind

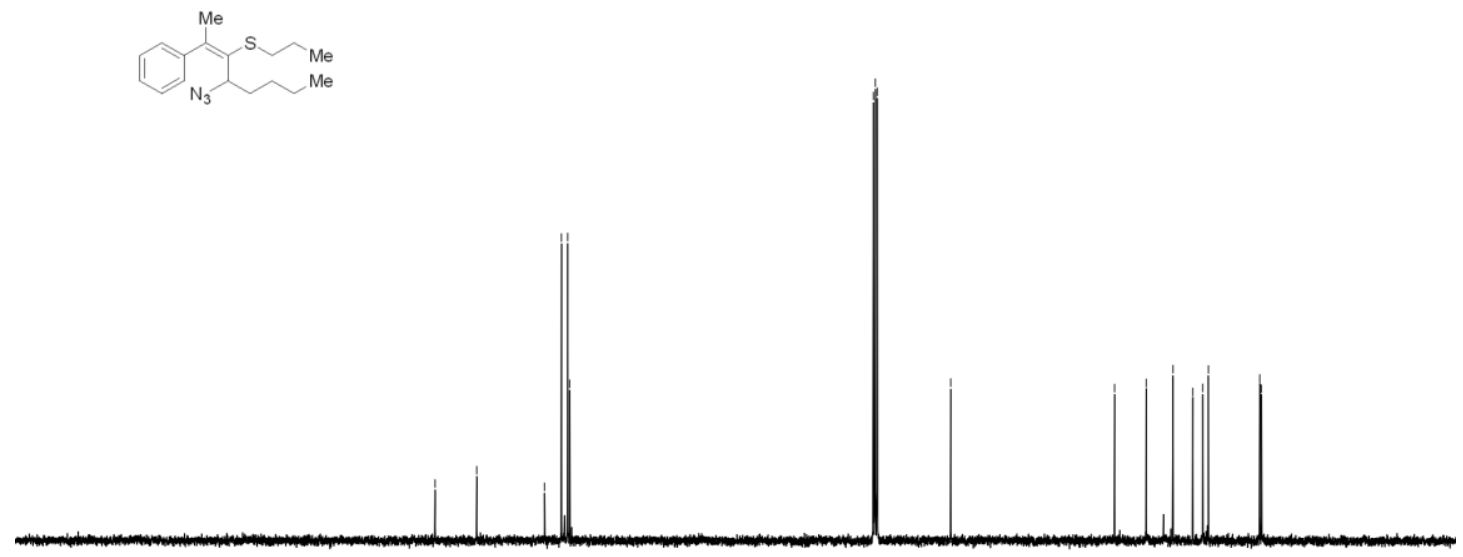

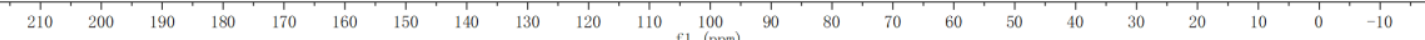


${ }^{1} \mathrm{H}$ NMR (400 MHz, $\mathrm{CDCl}_{3}$ ) spectrum of compound 4'

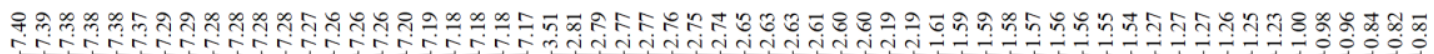

111
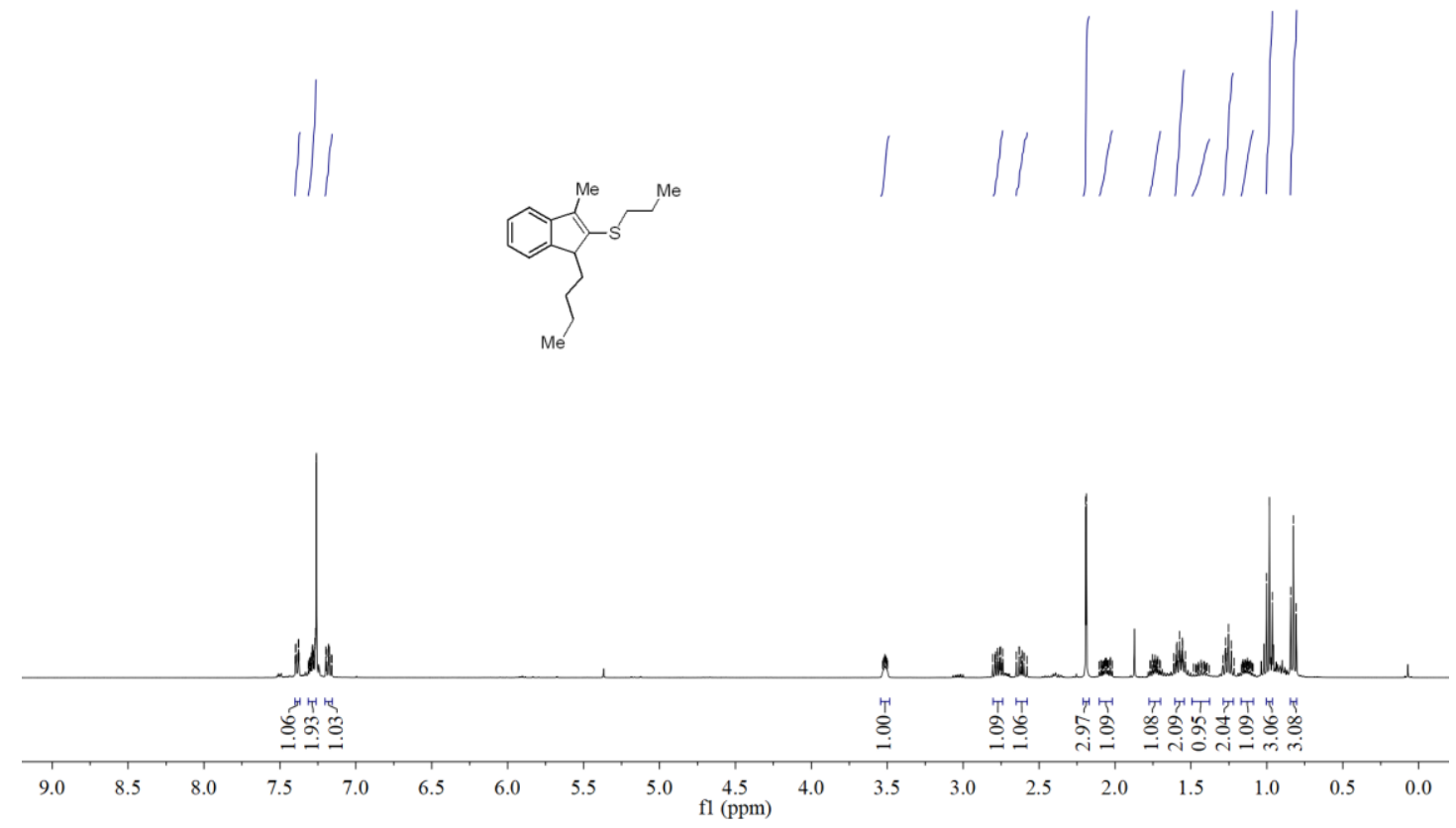

${ }^{13} \mathrm{C}$ NMR $\left(101 \mathrm{MHz}, \mathrm{CDCl}_{3}\right)$ spectrum of compound $\mathbf{4}^{\prime}$

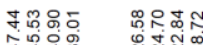

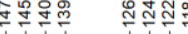

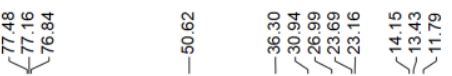

तh tr?

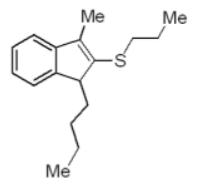

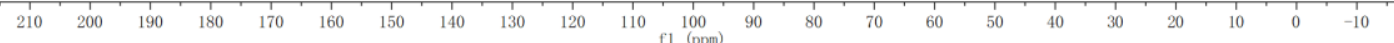

